University of Louisville

ThinkIR: The University of Louisville's Institutional Repository

Electronic Theses and Dissertations

8-2014

\title{
A novel diffusion tensor imaging-based computer-aided diagnostic system for early diagnosis of autism.
}

Mahmoud Mostapha

University of Louisville

Follow this and additional works at: https://ir.library.louisville.edu/etd

Part of the Computer Engineering Commons

\section{Recommended Citation}

Mostapha, Mahmoud, "A novel diffusion tensor imaging-based computer-aided diagnostic system for early diagnosis of autism." (2014). Electronic Theses and Dissertations. Paper 1015.

https://doi.org/10.18297/etd/1015

This Doctoral Dissertation is brought to you for free and open access by ThinkIR: The University of Louisville's Institutional Repository. It has been accepted for inclusion in Electronic Theses and Dissertations by an authorized administrator of ThinkIR: The University of Louisville's Institutional Repository. This title appears here courtesy of the author, who has retained all other copyrights. For more information, please contact thinkir@louisville.edu. 


\title{
A NOVEL DIFFUSION TENSOR IMAGING-BASED COMPUTER-AIDED DIAGNOSTIC SYSTEM FOR EARLY DIAGNOSIS OF AUTISM
}

\author{
By \\ Mahmoud Mostapha \\ B.S., Mansoura University, Egypt, 2009
}

A Thesis submitted to

J. B. Speed School of Engineering, University of Louisville in Partial Fulfillment of the Requirements for the Degree of

Master of Science

Department of Electrical and Computer Engineering University of Louisville

Louisville, Kentucky

August 2014 



\title{
A NOVEL DIFFUSION TENSOR IMAGING-BASED
} COMPUTER-AIDED DIAGNOSTIC SYSTEM FOR EARLY DIAGNOSIS OF AUTISM

\author{
By \\ Mahmoud Mostapha \\ B.S., Mansoura University, Egypt, 2009 \\ A Thesis Approved On
}

July 30th, 2014

\section{Date}

by the following Thesis Committee:

$\overline{\text { Ayman El-Baz, Ph.D., Thesis Director }}$

Tamer Inanc, Ph.D., Co-advisor

Dr. Guruprasad Giridharan 


\section{ACKNOWLEDGEMENTS}

In the Name of Allah, the Most Gracious, the Most Merciful. All the praises and thanks be to Allah Almighty, the Giver of bountiful blessings and gifts.

I would like to thank all those people that contributed to the completion of this thesis. I should also mention that this thesis would not have been possible without the help, support, guidance of my thesis advisor, Dr. Ayman El-Baz. I also express my deepest gratitude to Dr. Tamer Inanc, and Dr. Guruprasad Giridharan for being on my thesis committee with enthusiasm and taking interest in my research in the midst of many other responsibilities and commitments. And, I would like to thank Dr. Georgy Gimel'farb and Dr. Manuel Casanova for their useful discussions and valuable comments and feedback. I want to thank all the people who are part of the research group in the BioImaging Lab, Dr. Ahmed Elnakib, Dr. Fahmi Khalifa, Dr. Hisham Sliman, Ahmed Firjani, Matthew Nitzken, Ahmed Soliman, and Amir Alansary, who have become not only colleagues but also good friends. Finally, but most important of all, I am grateful to my parents, Mohamed Osman and Wessam Abo-Gabal, my brothers Ahmed, Ali, Yousef, and Abdalla, who have always given me their unconditional support, encouragement and advice, so that I could concentrate on my thesis.

I dedicate this thesis to the soul of my grandfather, Ali Abo-Gabal, who was my mentor and role model. He was and will always be source of inspration in my life and will always be in my heart guiding my steps. May Allah bless his soul, forgive him of his sins, make his grave a garden, and grant him the highest levels of paradise. 


\begin{abstract}
A NOVEL DIFFUSION TENSOR IMAGING-BASED COMPUTER-AIDED

DIAGNOSTIC SYSTEM FOR EARLY DIAGNOSIS OF AUTISM
\end{abstract}

\author{
Mahmoud Mostapha
}

August 12, 2014

Autism spectrum disorders (ASDs) denote a significant growing public health concern. Currently, one in 68 children has been diagnosed with ASDs in the United States, and most children are diagnosed after the age of four, despite the fact that ASDs can be identified as early as age two. The ultimate goal of this thesis is to develop a computer-aided diagnosis (CAD) system for the accurate and early diagnosis of ASDs using diffusion tensor imaging (DTI). This CAD system consists of three main steps. First, the brain tissues are segmented based on three image descriptors: a visual appearance model that has the ability to model a large dimensional feature space, a shape model that is adapted during the segmentation process using first- and second-order visual appearance features, and a spatially invariant second-order homogeneity descriptor. Secondly, discriminatory features are extracted from the segmented brains. Cortex shape variability is assessed using shape construction methods, and white matter integrity is further examined through connectivity analysis. Finally, the diagnostic capabilities of these extracted features are investigated. The accuracy of the presented CAD system has been tested on 25 infants with a high risk of developing ASDs. The preliminary diagnostic results are promising in identifying autistic from control patients. 


\section{TABLE OF CONTENTS}

Page

ACKNOWLEDGEMENTS

ABSTRACT

LIST OF TABLES

LIST OF FIGURES iii

iv

viii

ix

\section{CHAPTER}

$\begin{array}{llr}\text { I INTRODUCTION } & 1\end{array}$

A Overview of Medical Imaging Modalities . . . . . . . . . . . 2

1 Computed Tomography Imaging . . . . . . . . . . . . 4

$2 \quad$ Ultrasound Imaging $\ldots \ldots \ldots$

3 Nuclear Medicine Imaging . . . . . . . . . . . . 10

4 Magnetic Resonance Imaging . . . . . . . . . . . . 13

B Principles of Diffusion Tensor Imaging . . . . . . . . . . . 18

$1 \quad$ Diffusion Physics . . . . . . . . . . . . . . . 18

2 Diffusion-Weighted Imaging Acquisition . . . . . . . 20

3 Calculation of Diffusion Tensors . . . . . . . . . . 21

4 Diffusion Tensor Analysis and Measures . . . . . . . . 24

5 White Matter Fiber Tractography . . . . . . . . . . 30

6 Clinical Applications . . . . . . . . . . . 33

C The Proposed Computer-Aided Diagnosis System . . . . . . . . 35

D Thesis Organization . . . . . . . . . . . . . . . . 39

$\begin{array}{lll}\text { II } \quad \text { INFANT BRAIN SEGMENTATION } & 41\end{array}$

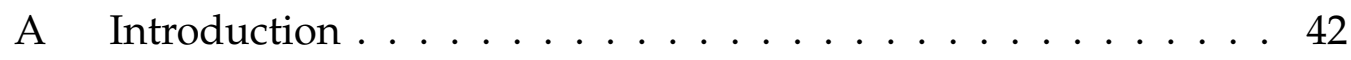


B Related Work . . . . . . . . . . . . . . . . . . . . 44

1 Probabilistic Segmentation . . . . . . . . . . . . . 44

2 Atlas-Based Segmentation . . . . . . . . . . . . . . . 46

3 Deformable Models-Based Segmentation . . . . . . . . 49

4 The Proposed Segmentation Framework . . . . . . . . 51

C Methods ...................... 54

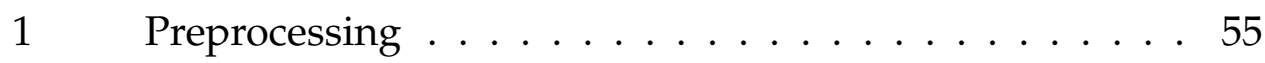

2 Brain Extraction . . . . . . . . . . . . . . 57

3 The Joint MGRF Segmentation Model . . . . . . . . . . 61

D Performance Evaluation Metrics . . . . . . . . . . . . 71

1 Dice Similarity Coefficient . . . . . . . . . . . . . . 71

2 Modified Hausdorff Distance . . . . . . . . . . . . . 73

3 Absolute Volume Difference . . . . . . . . . . . . . . 75

E Experimental Results and Evaluation . . . . . . . . . 76

1 Participants and Image Acquisition $\ldots \ldots \ldots$

2 Segmentation Results . . . . . . . . . . . . . . . . . 76

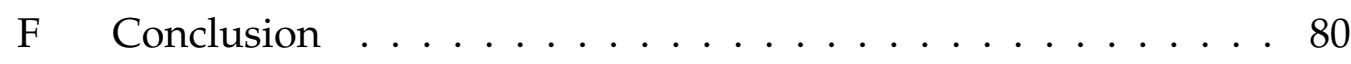

III INFANT BRAIN CLASSIFICATION 81

A Problem and Critical Unmet Need . . . . . . . . . . . . . 82

B Participants ................... 83

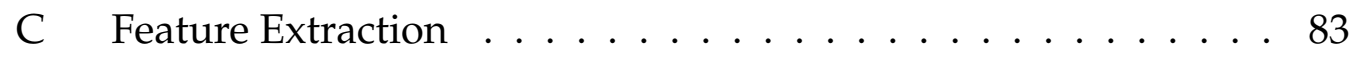

1 Shape Analysis . . . . . . . . . . . . . . . . . . 85

2 Connectivity Analysis . . . . . . . . . . . . . . . 92

D Classification ................... 96

IV CONCLUSION AND FUTURE WORK 98

A Contributions ..................... 99

B Future Work . . . . . . . . . . . . . . . . . . . . 101 
$\begin{array}{ll}\text { REFERENCES } & 103\end{array}$

$\begin{array}{ll}\text { APPENDIX } & 137\end{array}$

A Analytical Estimation of the bi-valued Gibbs Potentials 138

1 Unconditional Region Map Model . . . . . . . . . . . 139

2 Identification of the second-order MGRF model . . . . . 140

CURRICULUM VITAE 142 


\section{LIST OF TABLES}

TABLE

1 Accuracy of the proposed segmentation approach for each subject using dice similarity coefficient . . . . . . . . . . . . . 78

2 Accuracy of the proposed segmentation approach for each subject using the 95-percentile modified Hausdorff distance . . . . . . . . . 78

3 Accuracy of the proposed segmentation approach for each subject using the percentage absolute volume difference . . . . . . . . . . 78

4 Summary of the segmentation results represented by the mean \pm standard deviation values . . . . . . . . . . . . . . . . . . 79

5 Shape analysis results for control and autistic brains . . . . . . . . . . 90

6 Connectivity analysis results for control and autistic brains . . . . . . 94

7 Classification results using the five features that are extracted from the analysis of the diffusion tensor data . . . . . . . . . . . . 96 


\section{LIST OF FIGURES}

FIGURE Page

1 The four main classes of medical image modalities . . . . . . . . . . 3

2 Categories of medical image modalities . . . . . . . . . . . . 3

3 Examples of different types of medical images . . . . . . . . . . . 4

4 Different specialized computed tomography imaging acquisition techniques........................... 5

5 Different specialized ultrasound imaging acquisition techniques . . . 8

6 Different specialized nuclear medicine imaging acquisition techniques 11

7 Different specialized magnetic resonance imaging acquisition techniques ........................... 14

8 The two types of diffusion. . . . . . . . . . . . . . . . . . . . 19

9 An example of diffusion weighted imaging data collection with seven different weightings and gradient directions . . . . . . . . . 22

10 Graphical representation of diffusion tensor using the ellipsoid model 25

11 Various anisotropy and microstructural measurements obtained from diffusion tensor imaging . . . . . . . . . . . . 26

12 Different diffusion tensor imaging geometrical-based measurements 29

13 Color-coded orientation map . . . . . . . . . . . . . . . . . . . . . 29

14 An example of a full brain tractography . . . . . . . . . . . . . . . 30

15 Deterministic fiber tractography . . . . . . . . . . . . 32

16 An example of white matter structural variations associated with brain tumors . . . . . . . . . . . . . . . . . . . . 34

17 The main steps of a general computer-aided diagnostic system . . . . 37 
18 Block diagram of the proposed computer-aided diagnostic system for early diagnosis of autism spectrum disorders . . . . . . . . . 38

19 Comparison between infant and adult brains based on color coded orientation maps generated from diffusion tensor imaging . . . . . 43

20 The intensity histogram of different brain tissue classes using nondiffusion scan of an infant in the isointense stage . . . . . . . . . 52

21 The basic steps of the proposed framework for segmenting different structures from infant diffusion tensor brain data . . . . . . . . . 53

22 Examples of diffusion weighted imaging acquisition artifacts . . . . 55

23 The final estimated linear combination of discrete Gaussians density for each of the two classes . . . . . . . . . . . . . . . . 59

24 The discriminant threshold calculation method . . . . . . . . . . . 60

25 Illustration of the three-dimensional neighborhood system . . . . . 61

26 Illustration of the brain extraction procedure . . . . . . . . . 62

27 Block diagram of the proposed nonnegative matrix factorization based

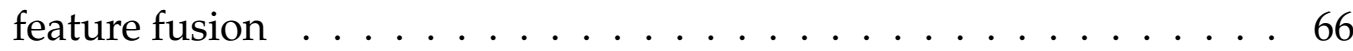

28 Illustration of the Euclidean distances used in calculating the nonnegative matrix factorization based probabilities . . . . . . . . 67

29 The shape prior probabilities estimated by the proposed adaptive shape model . . . . . . . . . . . . . . . . . 68

30 Samples of different pair-wise cliques for the used neighborhood

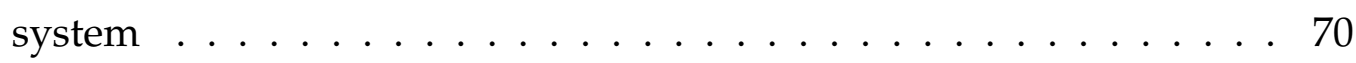

31 Diagram illustrating the meaning of segmentation errors used in calculating the dice similarity coefficient $\ldots \ldots \ldots \ldots . \ldots 73$

32 A schematic illustration for the Hausdorff distance calculation . . . 74

33 A schematic illustration for the absolute volume difference estimation 75

34 Three-dimensional visualization for the segmentation results . . . . 77

35 Segmentation results of the proposed approach . . . . . . . . . 79 
36 Illustration of the proposed feature extraction procedure . . . . . . . 84

37 Illustration of the spherical harmonics shape analysis steps . . . . . . 88

38 Error curves for the average autistic and control brains obtained for quantitative analysis of spherical harmonics . . . . . . . . . . . 89

39 Examples of spherical harmonic reconstructed brains compared to their original meshes . . . . . . . . . . . . . . . . . . . . . 91

40 Example of the extracted white matter fiber tracts . . . . . . . . 93

41 Boxplots representation of the connectivity analysis results . . . . . . 95 


\section{CHAPTER I}

\section{INTRODUCTION}

The latest advances in the field of medical imaging, which include magnetic resonance imaging (MRI), computed tomography (CT), and ultrasound (US), allowed the acquirement of images for almost all types and sizes of different structures with adequate degrees of contrast and resolution. Today, medical imaging can provide $3 \mathrm{D} / 4 \mathrm{D}$ functional as well as structural information about the scanned organ, which allows medical imaging to play a vital role in the composition of any current medical diagnostic tool. It has been efficiently used to support clinicians and radiologists in diagnosis, therapy decisions, and surgery operations. Compared to the other imaging modalities, MRI has become today the most powerful and dominant non-invasive tool for clinical diagnosis of different brain disorders (e.g., autism and dyslexia).

Based on the type of information that they provide, MRI modalities could be classified into two categories, namely functional and structural MRI. Diffusion tensor imaging (DTI) is a recent functional MRI method that characterize the threedimensional diffusion of water molecules in biological tissue using the diffusion tensor [1,2]. DTI has a wide range of clinical applications; it is used to examine normative white matter development, neurodevelopmental disorders (e.g., autism), and neurodegenerative disorders (e.g., amyotrophic lateral sclerosis) [3]. The research presented in this thesis is concerned with developing a novel computeraided diagnosis (CAD) system for the early diagnosis of autism spectrum disorders (ASDs). This thesis proposes a novel DTI-based CAD system for the early 
diagnosis of ASDs that integrates both shape and connectivity extracted features in their classification process. The proposed CAD system will enable the chance of early intervention for ASDs. With the new possibility of concentrated early intervention, the manifestation of the disorder could be lessened or even eliminated [4].

In this chapter, an overview of the current medical imaging modalities will be presented. Then, the main underlying principles of DTI will be explained in order to get a better in-depth understanding of this recent MRI imaging modality. Finally, the main components of the proposed CAD system will be introduced followed by the thesis overall organization.

\section{A Overview of Medical Imaging Modalities}

Medical imaging is the process of producing visual images that describe the interior of different areas inside the human body. Medical imaging procedures allow for noninvasive diagnosis of disease and monitoring of therapy, and can support medical and surgical treatment planning. For many diseases, early detection, more effective diagnosis, and improved monitoring of therapy through the use of imaging exams may possibly lead a morbidity reduction, added treatment choices, and improved life expectancy [5].

Each imaging modality has a different mechanism to provide the required physiological information of the organ being imaged. Therefore, each modality has a set of advantages and disadvantages, and the selection of the imaging technique must be tailored to the mandatory interest. Medical images can be classified based on their modalities (Figure 1) or based on the type of information that they provide (i.e., the structure or the function of the organ being imaged, see Figure 2). This section will provide an overview of familiar preoperative medical image modalities (see Figure 3). 


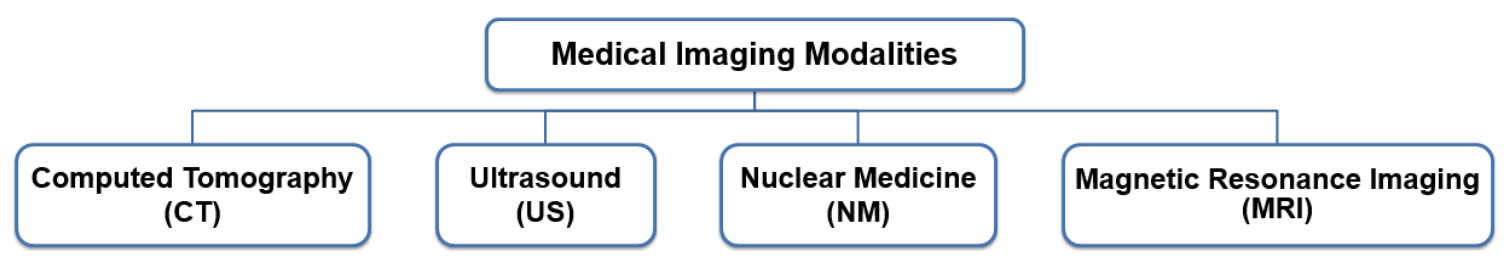

Figure 1: The four main classes of medical image modalities

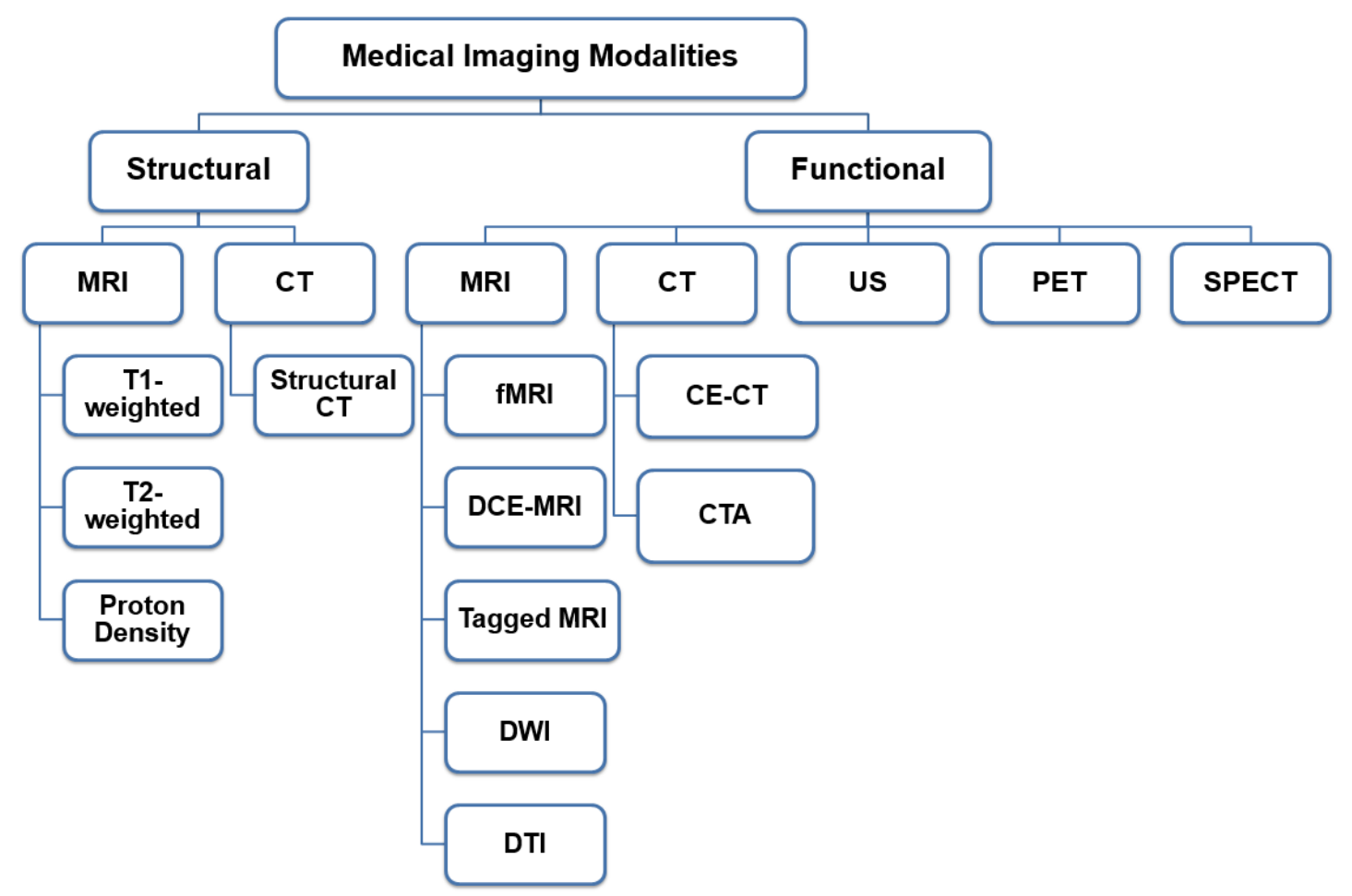

Figure 2: Categories of medical image modalities based on the type of information that they provide about the organ being imaged. 


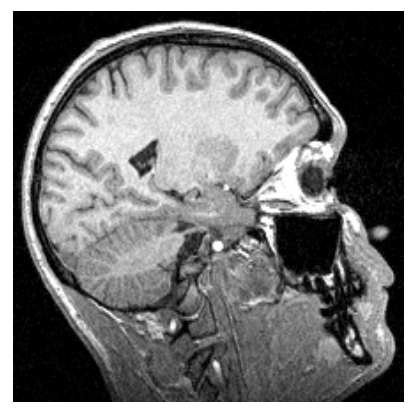

(a) Brain MRI image

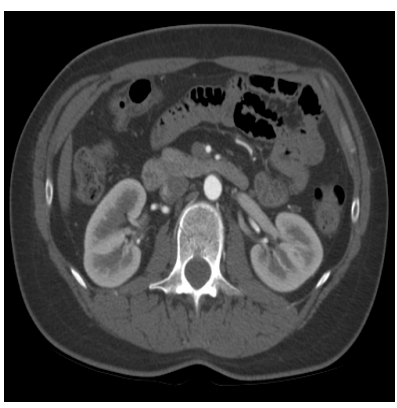

(b) Kidney CT image

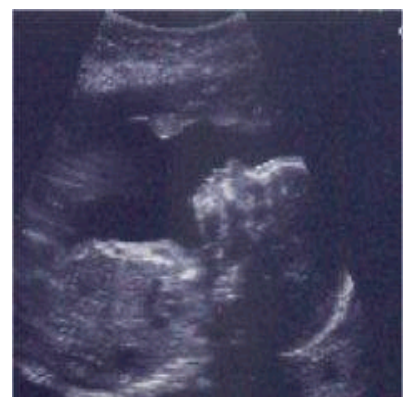

(c) Fetus US image

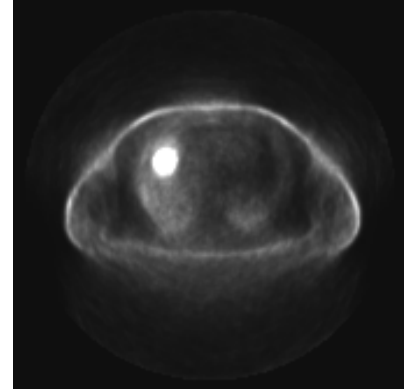

(d) Lung PET image

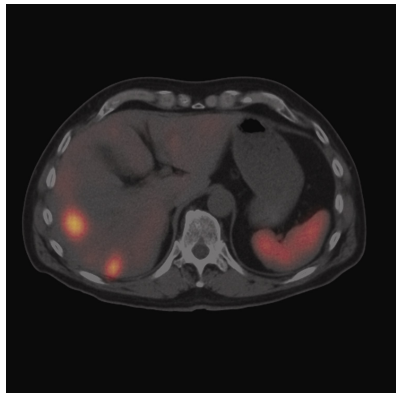

(e) Liver SPET image

Figure 3: Examples of different types of medical images.

\section{Computed Tomography Imaging}

Computed tomography (CT) has become one of the most common and valuable medical modalities in medical imaging since its introduction by the Nobel Prize winners Sir Godfrey Hounsfield and Alan McLeod McCormick. Even though also based on the variable absorption of X-rays by different tissues, CT imaging provides a different form of imaging known as cross-sectional imaging. The basic principle behind $\mathrm{CT}$ is that the internal structures of an object can be reconstructed from multiple measurements (projections) of $\mathrm{X}$-ray transmissions through the object. A CT image is typically called a slice, as it corresponds to a slice from a loaf of bread. The two-dimensional (2D) array of pixels (picture elements) in the CT image corresponds to an equal number of three-dimensional (3D) voxels (volume elements) in the patient. Each pixel on the $\mathrm{CT}$ image displays the average $\mathrm{X}$-ray attenuation properties of the tissue in the corresponding voxel. The most commonly 
used CT techniques are shown in Figure 4.

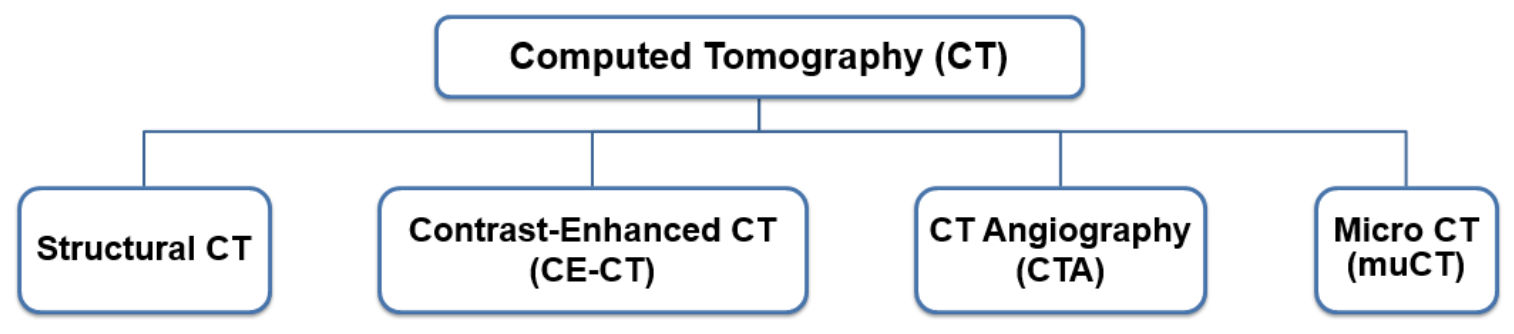

Figure 4: Different specialized CT imaging acquisition techniques.

Lately, images with much greater details could be obtained in a shorter scan time through the use of multi-detector (64 or more) CT scanners. Structural CT imaging modality has been comprehensively used in many clinical circumstances, such as determination of the extent of a trauma [6], localization of tumor [7], detection of lung diseases [8], diagnosis of heart disease [9], diagnosis of kidney diseases [10], study of dental problems [11], etc.

Although structural CT acquisition techniques provide structural (anatomical) information, they have a limited ability to provide any functional (physiological) information. The adminstration of contrast agents before a CT scan helps in reveling more functional information. Contrast-enhanced computed tomography (CE-CT) has been adopted for imaging different organs for disease diagnosis and pre-operative guidance and planning. The advantages of CE-CT include better contrast of anatomic structures, increased sensitivity for pathological lesions detection, and improved precision in lesion depiction. A distinct technique of CE-CT is functional CT, also called dynamic contrast-enhanced CT (DCE-CT). Functional CT has many clinical applications in brain and neck tumor diagnosis [12], lung nodule evaluation [13], examination of prostate cancer [14], and therapy monitoring [15].

CT angiography (CTA) is an optimized CT scan that is primarily concerned with producing pictures of blood vessels in the body. The advantages of CTA include the capability to perceive aneurysms (enlarged blood vessels), narrowed 
blood vessels, and impaired blood vessels, in an early stage for the determination of appropriate therapies. Moreover, CTA can reduce scan time, and lower cost compared with other comparable imaging techniques. CTA is used to diagnose the coronary artery disease of the heart [16], evaluate patients with acute ischemic stroke [17], identify renal artery stenosis [18], and detect acute pulmonary embolism [19].

The application of the CT was extended from the clinics to the research field. Microtomography or micro-CT (muCT), which is a distinct technique of $\mathrm{CT}$, was able to generate high resolution images (voxel spacing of $<100 \mu \mathrm{m}$ ) through longer exposure times [20]. Clinical applications of the micro-CT include small animal imaging [20,21], evaluation of mineralized tissues, such as insect exoskeletons [22] and skeletal tissues [23], quantification of pulmonary fibrosis and investigation of airway microstructures of animals lungs [24], assessment of bone and soft tissue disease and therapeutic response of small animals [25], and assessment of induced cardiac stress [26].

In conclusion, $\mathrm{CT}$ is more broadly used and faster than other imaging techniques, and may be less likely to require the person to be sedated or anesthetized. Moreover, CT scans provide good spatial resolution (the ability to distinguish between two structures at an arbitrarily small distance from each other) compared with MRI. In addition, CT cuts a lot of noise because it is computerized. Also, when compared to other imaging techniques like ultrasound, CT has a great penetration depth. On the other hand, the main concern of CT imaging is that the X-rays are ionized radiation, which may be harmful to patients and may induce cancer. However, recent $\mathrm{CT}$ technologies use low doses of radiation in order to reduce the radiation effects, but this usually comes with a reduced image quality. Overall, CT scan is a valuable tool that a physician can use in combination with other imaging modalities to reach the exact diagnosis for a patient. 


\section{Ultrasound Imaging}

The use of ultrasonography has been established in the medical field during the last decade of the twentieth century, and many scientific publications stated the lead of ultrasonography over commonly used X-ray techniques. Ultrasound (US) is the word used to refer to sound waves of frequencies above 20,000 Hertz $(\mathrm{Hz})$, beyond the range of human hearing. Frequencies of 130 megahertz $(\mathrm{MHz})$ are typical for US imaging. Lower frequencies between $3-5 \mathrm{MHz}$ are employed for abdominal areas such as the liver, as compared to $5-10 \mathrm{MHz}$ for small regions closer to the body surface, and 10-30 MHz frequencies are used for the skin or the eyes [27]. All different US techniques are based on the echo principle, where the time between the emission of a pulse and reception of its echo is used to estimate the distance between the transducer and the reflector. In addition, Doppler techniques could be used to describe the reflector's movements. The speed at which the waves travel is highly affected by various types of body tissues. By introducing additional mode to the ultrasound, the blood flow could be estimated. A Doppler probe inside the transducer gauges the velocity and direction of blood flow in the vessel by allowing sound waves to be audible. The loudness degree of the sound waves shows the level of blood flow in a blood vessel. Also, obstruction of blood flow could be determined by the absence of these sounds. A hierarchy of the different modes/types of each of the two categories is presented in Figure 5.

A-mode (A-scan, amplitude modulation) is a one dimensional (1D) scan, which is infrequently used due to the limited information provided (distance measurements only). Clinical applications of A-mode scans include the measurement of eye dimensions prior to corneal thinning or lens replacement [28], the localization of solid masses in the eye orbit or globe [29]. In B-mode (brightness modulation), echoes are displayed as points of different grey-scale brightness. M-mode or TM-mode (time motion) is primarily a continuous series of B-mode scans. The main objective of M-mode scan is to provide the degree of movement of different 


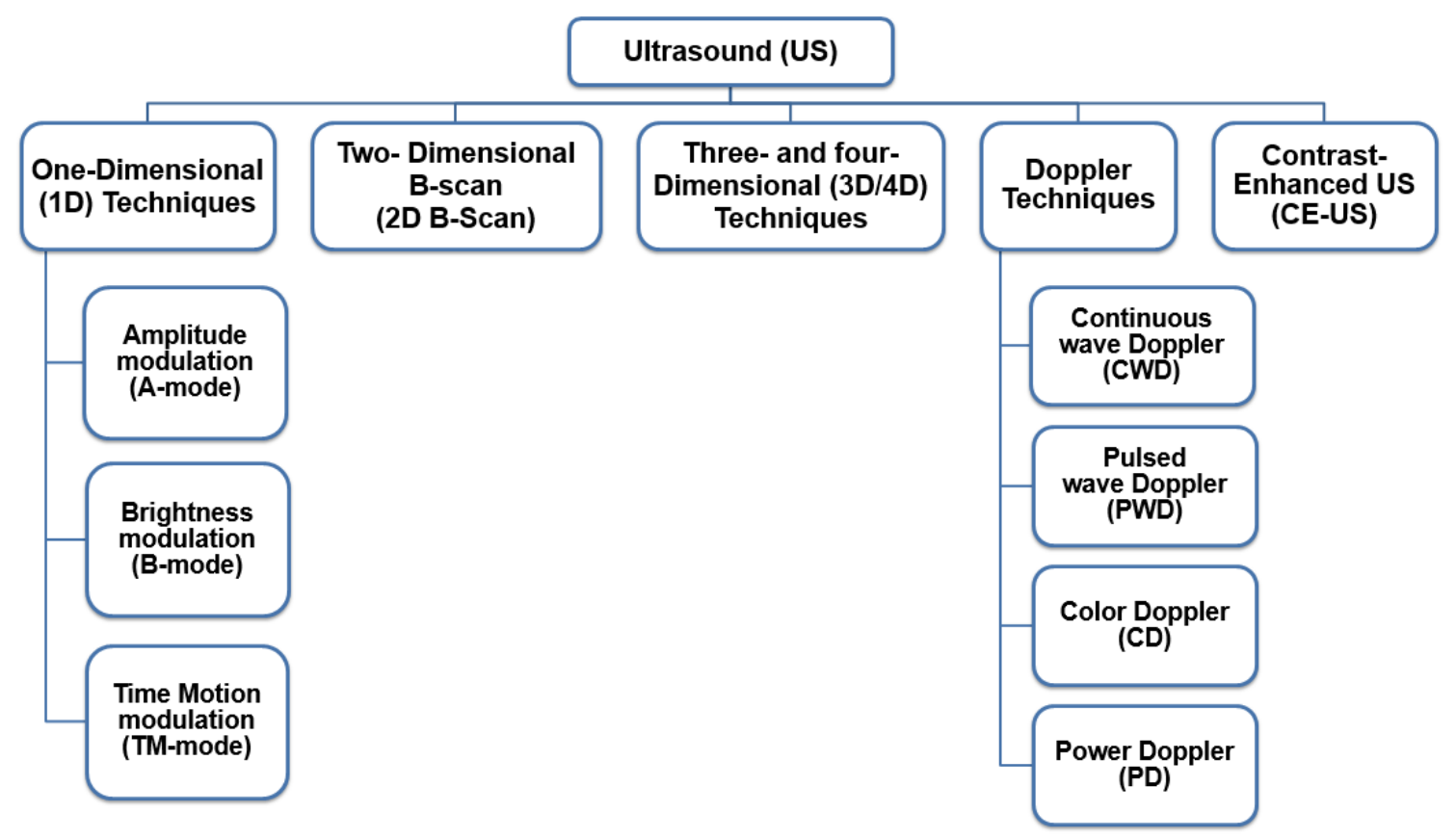

Figure 5: Different specialized ultrasound imaging acquisition techniques for both anatomical imaging and for blood velocity measurements.

scanned tissues within the body. Applications of M-mode scans include the detection of heart valves and heart wall motion [28], the detection of valvulopathies (calcification, etc.) and cardiomyopathies (dyskinesis, aneurysm, etc.), and the measurement of abdominal muscle thickness [30].

Two-dimensional B-scan (2D B-scan) is an extension from the 1D B-scan using an arrangement of many one-dimensional lines in one plane. 2D B-scans are typically used to study both stationary and moving structures. Applications of Bmode scans include the study of atherosclerotic progression [31], characterization of carotid artery plaques [32], and cardiac imaging [28]. Recently, 3D ultrasound images have been introduced for anatomical imaging and diagnostics. Although the 3D ultrasound is still not well-established, it has already shown promise in many medical applications, such as the study of the abdomen and pelvis and imaging of fetal and uterine malformation [28], measuring the kidney long axis [33], and the estimation of the dimensions of cardiac valves [28]. In order to track the move- 
ment of the organ of interest, recent advances attempt to obtain 4D US scans by acquiring multiple 3D image frames in a short period of time [33].

In US Doppler techniques, the Doppler effect is used to provide further information that is specially used to examine blood flow. Continuous wave Doppler (CWD) as the name implies, involves a continuous generation of US waves using a transmitter transducer coupled with a continuous US wave reception using another specific transducer dedicated for receiving the US waves. The main drawback of the CWD is its lack of sensitivity and depth discrimination. On the other hand, the main advantage of the CWD, is its ability to measure high blood velocities at any depth (e.g., the highest velocities in any valvular). Pulsed wave Doppler (PMD) is a more data-selective method than the CWD mode. It is typically used to provide a localized velocity measurement over a small area. The main drawback of PWD is that it has a limit for the highest frequency that can be measured due to what is technically known as aliasing. In addition, PWD can not accurately measure high blood flow velocities encountered in certain types of valvular and congenital heart disease. Color Doppler (CD) and power Doppler (PD) displays are used as duplex systems integrated into the B-scan image [27]. In CD method, the flow map is superimposed within the high-resolution image, Doppler shifts are measured in a few thousand sample volumes located in an image plane, and a color is associated with each velocity and displayed on the location of the volume on top of the B-mode image. PD is based on the combined power of the Doppler signal, and is five times more sensitive in detecting blood flow than CD; however, it provides no information about the flow direction [27]. To enhance the quality of the US image, a contrast-agent can be administrated before capturing the US image. Contrast-enhanced US (CE-US) imaging is used to further investigate the physiological basis of diseases. Applications of CE-US include imaging the blood perfusion in organs, measurement of the blood flow rate in the heart and other organs, and imaging the liver to detect hepatocellular carcinoma [34]. 
To conclude, US imaging has been involved in many applications. However, US drawbacks include its low image quality and thus its inability to image the fine details of the structures. Moreover, it is limited by the inability to image through air (lungs, bowel loops) or bone. Even in the absence of bone or air, the depth of penetration of US is limited by its frequency. In addition, the ultrasound images are highly operator-dependent and are affected by the patient body habitus. For example, larger patients may have a decrease in image quality due to sound wave absorption in the subcutaneous fat layer. On the other hand, the main advantage of ultrasound imaging in medical image diagnosis is that it offers a safe imaging technique, which involves no ionizing radiation. This makes US imaging the modality of choice for many applications such as gynecology and obstetrics. Moreover, US is fairly inexpensive and can acquire real time images with a high frame rate. Furthermore, recent advances in US instruments have made them more small, portable, popular, and usable. These features, and the constant developments in image quality and resolution have extended the use of US to many areas in medicine beyond customary diagnostic imaging.

\section{Nuclear Medicine Imaging}

Today, there are between 15 and 20 million nuclear medicine (NM) scans performed annually in the USA. During an NM scan, a slight quantity (nanogrammes) of radioactive material (radiotracer) is inserted into the patient. Eventually, the agent will mount up in a certain body organ, and the healthiness of the tissue can be assessed using several aspects, which include the accumulation position, quantity and speed. Since NM are able to show the spatial distribution of metabolic or biochemical activity in the body, it is an important complement to MRI, CT and US. Typical NM imaging modalities are shown in Figure 6.

Scintigraphy is a $2 \mathrm{D}$-imaging modality that produces a picture of radioactive material (radiopharmaceuticals) distribution within the human body. The 


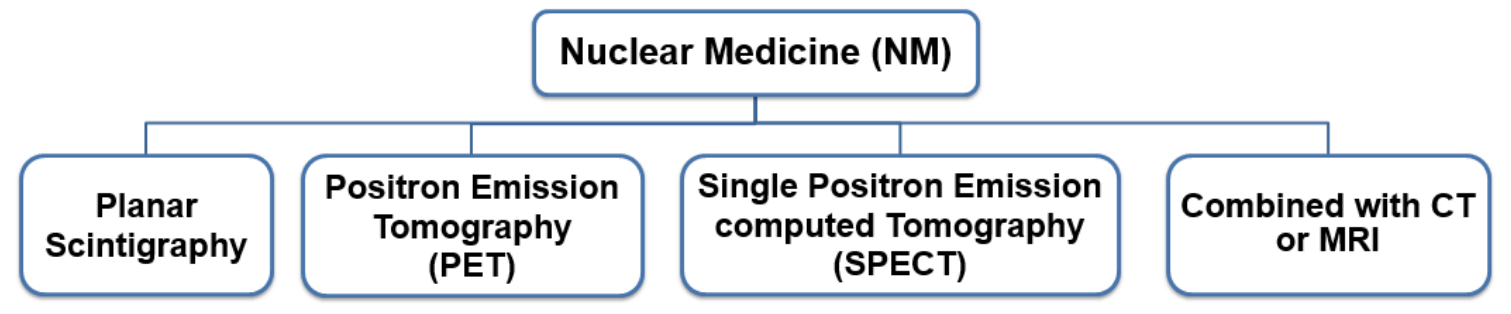

Figure 6: Different specialized NM imaging acquisition techniques.

main clinical application of planar scintigraphy is whole-body bone scan to detect bone fractures as areas of increased radiation. Also, it can be helpful to detect certain types of abnormalities that are difficult to detect with other imaging modalities. For example, lesions or malignant tumors can be visually detected in planner scintigraphy as areas of concentrative distribution of radiation (brighter than normal) or areas of absence of concentration (darker than normal). Another application of planner scintigraphy is to image the brain. It is usually used to detect malignant tumors, indicated by higher uptake of radiation, since the blood flow is often higher in the malignant tumor than other tissue [28]. In addition, it is used to confirm brain death as the carotid arteries are visualized cut off at the base of the skull [28].

Positron emission tomography (PET) is an NM imaging technique that produces a 3D image that provides functional information about specific processes in body. The gamma camera in the PET imaging technique detects pairs of gammarays, that follow the annihilation of an emitted-positron by a positron-emitting radionuclide. Although PET imaging is known for its high cost, it is often chosen to produce better image quality and resolution. PET has been involved in many medical applications. For example, FDG-PET has been widely used in oncology applications to explore the possibility of cancer metastasis. Other positron-emitting radionuclides are also used in PET to image the tissue concentration of many other types of molecules of interest. For example, PET imaging with Oxygen-15 radioactive molecule is used in brain imaging in order to measure regional blood flow [28]. 
In addition, PET has many applications in neurology. Particularly, PET imaging is used in brain tumor evaluation, early identification of recurrence, and in assessing neuro-degenerative disorders such as Alzheimer's. PET imaging can also be used in cardiac studies to measure the blood flow and the metabolism inside the heart. Applications of cardiac PET include the assessment of myocardial fatty acid metabolism and myocardial infarction. In addition, cardiac PET can be used to determine the need for a heart transplant or a bypass surgery [28].

Single positron emission computed tomography (SPECT) is another NM technique, which have the same basic principle of conventional planner scintigraphy. However, instead of acquiring a 2D image, it produces a 3D tomographic scan from a series of 2D nuclear medicine images from adjacent slices of tissue. Application of SPECT images include measuring the blood perfusion in the brain, which is clinically beneficial as indication of strokes or brain diseases like Alzheimer's [28]. Like the planner scintigraphy, SPECT images can also be used for bone scanning and tumor detection. In addition, SPECT images can be used to measure myocardial perfusion and blood flow patterns in the heart to detect coronary artery disease and myocardial infarct [28].

To provide anatomy information in addition to the functional information of nuclear imaging, dedicated combined devices are designed to capture PET/CT, SPECT/CT, and PET/MRI images sequentially, within same session from the patient, and to combine them into a single superposed (co-registered) image using image fusion or registration. PET/CT imaging technique has been widely considered in lung cancer applications to add precision of anatomic localization to functional imaging. In addition to PET/CT, PET/MRI is a hybrid imaging modality that incorporates MRI soft tissue imaging and PET functional imaging. Finally, SPECT/CT is a hybrid imaging modality that incorporates CT anatomical imaging and PET functional imaging in order to add the precision of anatomic localization to functional imaging. For example, SPECT/CT is useful in locating ectopic 
parahyroid ademomas, which may not be in their usual locations in the thyroid gland.

In conclusion, NM offers unique information, both functional and structural, which often no other imaging modality can provide. In addition, NM allowed for the detection of many abnormalities in an early stage, which can lead to better treatment and more successful outcomes. Moreover, NM images have very high sensitivity and specificity due to the absence of any natural radioactivity from the scanned body. On the other hand, compared to other imaging modalities, NM scans (especially scintigraphy and SPECT) have poor signal-to-noise ratio (SNR), low spatial resolution ( 5-10 $\mathrm{mm}$ ) and long image acquisition times [35].

\section{Magnetic Resonance Imaging}

Compared to the other imaging modalities, magnetic resonance imaging (MRI) is the most recently developed, and has become the most powerful and dominant non-invasive tool for clinical diagnosis of different diseases [36]. The fundamental principle of MRI is based on the use of a strong static magnetic field in which the hydrogen nuclei (single proton) of water molecules in human tissues are aligned parallel to that field. Then, an external radio frequency (RF) pulse (wave) is applied to the unpaired magnetic spins (proton) aligned in the static magnetic field, making them spin in different directions, and the acquired image intensity in any spatial location will depend on the total number of protons as well as physical properties of the tissue such as viscosity, stiffness and protein content [37]. The most commonly-known specialized MRI techniques are shown in Figure 7.

The amount of signal strength on the MRI is mainly governed by the magnetic relaxation properties of the scanned body's atomic nuclei. It is essential to note that there are two relaxation times which govern the return to the equilibrium magnetization state. These are referred to as the longitudinal relaxation time (T1) and transverse relaxation time (T2), based on the orientation of the component 


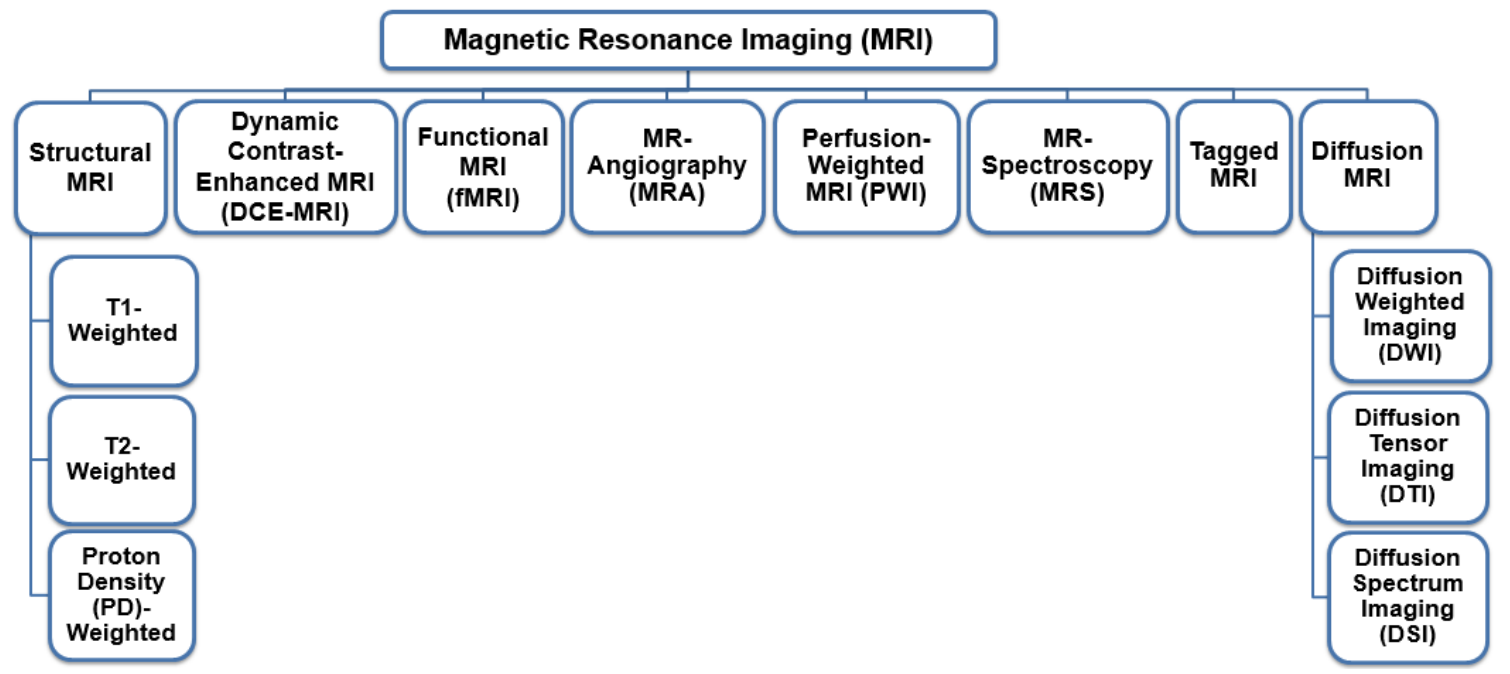

Figure 7: Different specialized MRI acquisition techniques.

with respect to the magnetic field. The water and macromolecules dissimilar levels of protons, in various tissues, cause their T1 and T2 values to be different. T1weighted MRI is a commonly-run clinical MRI scan that emphasizes T1-contrast, and is known to be the best MRI technique for demonstrating anatomical details. T2-weighted scans are another elementary type that stresses T2 contrast. Typically, T2-weighted scans are used to show high dissimilarity between fluid, abnormalities (e.g., tumors, inflammation, trauma), and the surrounding tissues. Finally, since the proton density (PD) is a fixed physical value, PD-weighted images have no T1-weighting nor T2-weighting. The main advantage of the PD-weighted images is the better contrast between fluid and non-fluid tissues. However, PDweighted images typically show less contrast resolution when compared to T1- or T2-weighted images. In general, the dynamic nature of MRI allows the optimization possibility for it to suite any specific study in which a specific anatomical part or disease process is targeted. For example, the signal intensities of the imaged tissues can be controlled by selecting the type of the scan: either proton density, T1-weighted, or T2-weighted, and for a given type of scan, a pulse sequence is designed and imaging parameters are optimized to produce the desired image 
contrast $[36,38,39]$.

Even though structural MRI offers superb soft tissue contrast, it lacks functional information. Functional MRI (fMRI) is a technique to conclude which parts of the brain are involved in specific mental tasks as well as common brain operations such as speech, language and sensory motion. The foundation of fMRI is that MRI signal intensity varies based on the level of oxygenation of the blood in the brain [40]. In fMRI, sequential images are acquired, one while the brain is in rest state followed by another one after the brain is stimulated in some way. Various different kinds of stimulus can be used: visual-, motor- or auditory-based. The images' intensity changes between different scans are usually small $(0.2-2 \%)$ using a 3 Tesla scanner, and so experiments are repeated a number of times with rest time (baseline) between each stimulation cycle. Different from Electroencephalography (EEG) that provides surface information (brain waves) through electrodes mounted on the patients' scalp, fMRI has the advantage of providing in-depth details about the inside the brain. Clinical applications of fMRI include epilepsy surgery [41], and diagnosis of schizophrenia [42] and cerebral injury [43]. Dynamic contrast-enhanced MRI (DCE-MRI) is another MRI technique, which has the capability to deliver greater information of the anatomy, function, and metabolism of targeted tissues [44]. The technique involves the acquisition of serial MR images with high temporal resolution before, during, and at several times after the administration of a contrast agent into the blood stream. DCE-MRI is frequently used to increase the contrast between different tissues, mainly normal and pathological. It has been widely used in several clinical applications, including finding of pathological tissue, e.g., brain tumors [45], analysis of myocardial perfusion [46], early detection of acute renal rejection [47-53], and detection of prostate cancer [54,55].

Distinct from X-ray angiography, magnetic resonance angiography (MRA) is noninvasive as it does not need the usage of contrast agents, even though they can be used to enhance the signal dissimilarity between flowing blood and tissue. 
The two most common MRA techniques are time-of-flight (ToF) and phase contrast (PC). Both rely on different physical effects, and so will result in scans with different information about the vasculature [56]. Specially, PC-MRA suppresses background signals and quantifies blood flow velocity vectors for each voxel. While TOF-MRA is less quantitative, it is fast and offers high contrast images. Perfusionweighted imaging (PWI) is a serial MRI technique designed to image blood flow into brain vasculature. PWI uses an MR contrast to provide metabolic and hemodynamic information in the first few hours post-stroke [57]. The most common PWI technique is dynamic susceptibility contrast (DSC) imaging. DSC has been comprehensively designed to quantify the cerebral blood flow of the brain for patients with vascular stenosis [58], stroke [59], and brain tumors [60].

Magnetic resonance spectroscopy (MRS) is an MRI technique that provides important information about the biochemical changes within cells and can also be used to detect the size and stage of a tumor. Different from conventional MRI, MRS detects the resonance spectra of chemical compounds other than water [61]. MRS has been investigated for identification of patients with cancerous conditions in the brain [62], prostate [63, 64], breast [65, 66], cervix [67, 68], pancreas [69], and esophagus [70]. Tagged MRI is a well known technique for comprehensive and noninvasive visualization of cardiac motion and deformation throughout the heart cycle [71]. Cardiac MRI tagging places a pre-specified pattern of temporary markers (called tags) inside soft body tissues (e.g., tag lines) created by patterns of magnetic spin in the examined tissue so that the motion in the tagged tissue can be measured from the images [72]. Whereas customary MRI can provide information about the motion at the boundaries of an object, the tag lines allow the chance to examine the strain and displacement of the interior of the tissue in more detail [73]. Therefore, tagged MRI is used to localize heart diseases (e.g., coronary atherosclerosis) and global conditions (e.g., heart failure and diabetes) that cause heart wall dysfunction. 
Diffusion MRI first came into existence in the mid-1980s, and it is a functional technique that is based on the measurement of micromovements (random, Brownian) of extracellular water molecules inside the body. These movements provide indirect information about the structures surrounding these water molecules. Diffusion MRI is a non-invasive technique that has the advantage of being acquired very rapidly, without the use of any intravenous contrast material or specialized hardware. Diffusion MRI can be classified into three main categories, namely, diffusion-weighted imaging (DWI), diffusion tensor imaging (DTI) and diffusion spectrum imaging (DSI). DWI is a well-established MRI technique that has been effectively used for tumor localization and diagnosis [74], investigation of brain disorders, such as epilepsy, multiple sclerosis, brain abscesses, brain tumors, and hypertensive encephalopathy [75], and in-vivo study of aspects of tissue microstructure [76]. DTI is another type of diffusion MRI, which is based on the measurement of the Brownian motion of water molecules in tissue. DTI is a recent MRI technique used to study in-vivo tissue microstructure, such as the connectivity between different brain areas. This MRI modality allows the scientist to be able to look at the network of nerve fibers. Nowadays, DTI has been investigated by neuroscientists to study a number of disorders (e.g., addiction, epilepsy, traumatic brain injury, and various neurodegenerative diseases) and to demonstrate subtle abnormalities in a variety of diseases, (e.g., stroke, multiple sclerosis, dyslexia, autism, and schizophrenia) [75-80]. Although DTI has been proven as a valuable MR method in studying in-vivo fibrous connectivity, it cannot directly image multiple fiber orientations [81]. In order to overcome this drawback, DSI has been developed to image complex distributions of intravoxel fiber orientation. Therefore, DSI permits better mapping of axonal trajectories than other diffusion imaging approaches [81]. DSI drawbacks include the additional hundreds of images needed and the longer acquisition time when compared with DWI and DTI [82].

To conclude, the advantages of MRI includes the absence of any ionizing 
radiation, the possibility of acquiring two- or three-dimensional plane images, the excellent contrast of soft-tissue, a high spatial resolution $(<1 \mathrm{~mm})$, and the insignificant penetration effects. In addition, MRI can highlight different components of the scanned area using different pulse sequences: a preselected strength, shape, and timing of defined RF and gradient pulses (external fields). On the other hand, MRI suffers from slower image acquisition (a typical MRI clinical protocol might last 30-40 minutes with several different types of scan being run) when compared to CT and US. Also, the number of patients who are prohibited from MRI scans due to metallic implants from previous surgeries cannot be ignored. In addition, MRI systems are relatively higher in cost when compared to CT and US equipments [35]. Also, MRI suffers from sensitivity to noise and image artifacts, and its signals are dependent on the imaging sequence used and can become non-linear beyond certain concentrations leading to errors in extracted physiology. Nevertheless, recent improvements in MRI design aim to aid claustrophobic patients by using more open magnet designs and shorter exam times. However, there is often a trade-off between image quality and open design.

Since this thesis is concerned with building a DTI-based CAD system for the early diagnosis of ASDs, a brief overview of the basic principles of DTI is presented in the next section.

\section{B Principles of Diffusion Tensor Imaging}

\section{Diffusion Physics}

Diffusion is the kind of motion that has nothing to do with physiological motion, but it is due to the random motion of molecules due to thermal energy. This motion is also called intra-voxel incoherent motion (IVIM) or Brownian motion [83]. Water diffusion in biological tissues occurs inside, outside, around, and through cellular structures. The diffusion of water is hindered by cellular mem- 
branes causing the existence of more tortuous paths, and the diffusion tortuosity could be amplified in the case of cellular swelling or increased cellular density. On the other hand, intracellular water have a tendency to be more restricted by cellular membranes, and both hindered and restricted diffusion lessen water apparent diffusivity [80]. The research presented by this thesis is mainly concerned with the human brain tissues, which could be divided into three categories, white matter (WM), grey matter (GM) and cerebrospinal fluid (CSF). Water molecules in an unconstrained medium, such as in CSF regions, diffuse along any orientation in a similar manner. This is called isotropic diffusion (Figure 8 (a)). On the other hand, molecular diffusion in WM is more restricted by the WM tracts, and the restrictions depend on the tract spatial orientation. Parallel to the fiber track, water molecules can diffuse more freely than perpendicular to it, and the diffusion becomes anisotropic with a privileged direction (Figure $8(\mathrm{~b})$ ). As a result, the water diffusion in that case could be used to reflect information about the underlying anatomical architecture of brain tissues [83].

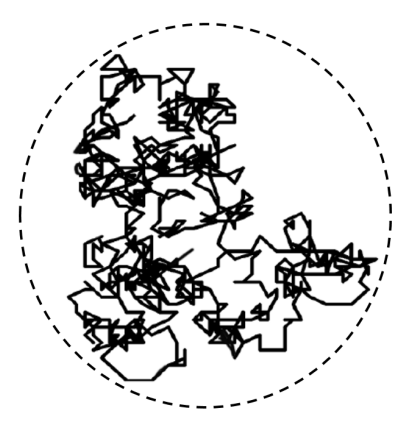

(a) Isotopic diffusion

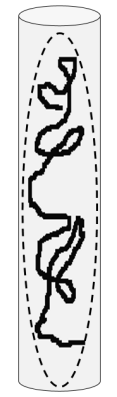

(b) Anisotropic diffusion

Figure 8: The two types of diffusion. In isotopic diffusion, motion occurs equally and randomly in all directions (e.g., the brain ventricles). While, anisotropic diffusion in a confined medium has motion oriented along one direction more than the other directions (e.g., the brain white matter tracts).

Water molecules diffusion is modeled using a probability density function $P(\mathbf{r}, \mathbf{t})$ which gives the existence probability of molecule in a certain position $\mathbf{r}=$ $[x, y, z]$ at a particular time $t$. In three dimensions, the diffusion Equation is given 
by [84]

$$
\frac{\partial P(\mathbf{r}, t)}{\partial t}=\nabla \cdot(\mathbf{D} \nabla P(\mathbf{r}, t)),
$$

where $\mathbf{D}$ is the diffusion coefficient $\left(\right.$ in $\mathrm{s} / \mathrm{mm}^{2}$ ), a $3 \times 3$ symmetric positive semidefinite matrix, and in the case of free diffusion (isotropic medium), $\mathbf{D}=4 d \pi \mathbf{t}^{3 / 2} \mathbf{I}_{3 \times 3}$, where I is identity matrix, and $d$ is a positive diffusion constant [85]. The isotropic case solution to Equation (1) is found to be a Gaussian distribution, and is given by

$$
P(\mathbf{r}, t)=\frac{1}{\sqrt{4 \pi t d}} \exp \frac{-\|\mathbf{r}\|^{2}}{4 t d} .
$$

In case of an anisotropic medium, the solution of Equation (1) is given by

$$
P(\mathbf{r}, t)=\frac{1}{(4 \pi t)^{\frac{3}{2}}|\mathbf{D}|^{\frac{1}{2}}} \exp \frac{-\mathbf{r}^{T} \mathbf{D}^{-1} \mathbf{r}}{4 t},
$$

where $\mathbf{D}$ is called the diffusion tensor matrix. In the general case of a fiber that is oriented arbitrarily and has different diffusion coefficients along different directions, the off-diagonal elements of $\mathbf{D}$ appear as follows:

$$
\mathbf{D}=\left(\begin{array}{ccc}
D_{x x} & D_{x y} & D_{x z} \\
D_{y x} & D_{y y} & D_{y z} \\
D_{z x} & D_{z y} & D_{z z}
\end{array}\right) .
$$

The diffusion tensor defines the covariance of diffusion displacements in 3D normalized by the diffusion time. The matrix $\mathbf{D}$ is symmetric: $D_{y x}=D_{x y}, D_{z x}=$ $D_{x z}$, and $D_{z y}=D_{y z}$; therefore, the tensor matrix has six independent elements (or six degrees of freedom), which describe the strength of the diffusion (three degrees of freedom) and the spatial orientation of the tensor (another three degrees of freedom).

\section{Diffusion-Weighted Imaging Acquisition}

Currently, the most common DWI acquisition approach is single-shot, echo planar imaging (SS-EPI) sequences [83]. SS-EPI uses only one RF excitation to ac- 
quire the whole set of planar k-space measurements required to construct a DWI image, which reduces the acquisition time significantly when compared to the conventional pulsed-gradient, spin echo (PGSE) pulse sequence that requires the use of several RF/phase selection repetitions [86]. Although, SS-EPI technique is considered the most common pulse sequence currently used, it suffers from several drawbacks, including magnetic field inhomogeneities, eddy currents, and signal loss from dephasing. Some methods that are usually used to mitigate these effects are described in [86].

According to Stejskal and Tanner [87], the relationship between a measurement without diffusion weighting $S_{0}$ and one with diffusion weighting $S$ is given by

$$
S_{i}=S_{0} \exp -b D
$$

where $b$ is the diffusion weighting factor ( $b$ value) [88], which is defined as

$$
b=\gamma^{2} \delta^{2}\left(\Delta-\frac{\delta}{3}\right)|\mathbf{g}|^{2},
$$

where $\gamma$ is the Lamor constant (42 MHz/ Tesla), $|\mathbf{g}|$ is the strength of the diffusion sensitizing gradient pulses, $\delta$ is the gradient pulse duration, and $\Delta$ is the time between diffusion gradient RF pulses.

The diffusion values, $D$ are also called apparent diffusion components (ADC) values to stress the point that the diffusion values produced from depend on the changing experimental conditions, such as the direction of the sensitizing gradient and other sequence parameters ( $\delta$ and $\Delta$ ) [89]. Therefore, DWI is very sensitive to the choice of the scanner acquisition parameters, raising the need to develop a new modality like DTI, the next advanced generation of DWI.

\section{Calculation of Diffusion Tensors}

In the special case of anisotropic medium, the general form of Equation (5) could be written as 


$$
S_{i}=S_{0} \exp -b \hat{\mathbf{g}}_{i}^{T} \mathbf{D} \hat{\mathbf{g}}_{i}
$$

where $\hat{\mathbf{g}}=\mathbf{g} /|\mathbf{g}|$ is the normalized gradient vectors.

Since the diffusion tensor D has six degrees of freedom, six diffusion constants along six independent axes should be measured. Therefore, at least seven diffusion-weighted images are needed (six measurements taken from different gradient directions, in addition to the intensity corresponding to $S_{0}$ (often called b0 image)). An example of data collection of seven images with different diffusion weightings and gradient directions is shown in Figure 9.

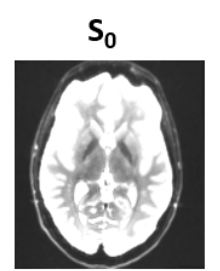

$$
\mathrm{g}_{0}=\left(\begin{array}{l}
0 \\
0 \\
0
\end{array}\right)
$$

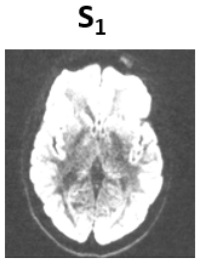

$\mathrm{g}_{1}=\left(\begin{array}{l}1 \\ 1 \\ 0\end{array}\right)$

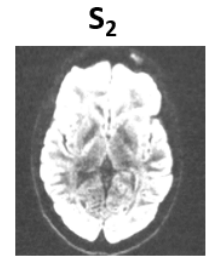

$\mathrm{g}_{2}=\left(\begin{array}{l}0 \\ 1 \\ 1\end{array}\right)$

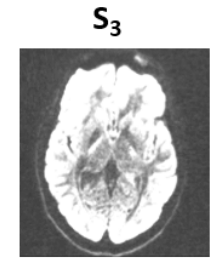

$\mathrm{g}_{3}=\left(\begin{array}{l}1 \\ 0 \\ 1\end{array}\right)$

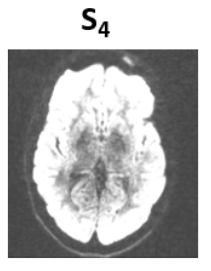

$\mathrm{g}_{4}=\left(\begin{array}{c}0 \\ 1 \\ -1\end{array}\right)$

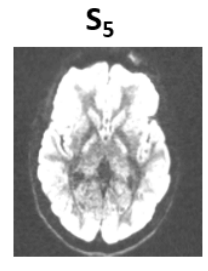

$g_{5}=\left(\begin{array}{c}1 \\ -1 \\ 0\end{array}\right)$

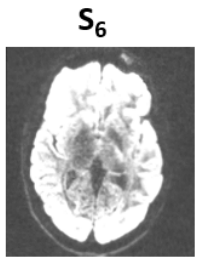

$\mathrm{g}_{6}=\left(\begin{array}{c}-1 \\ 0 \\ 1\end{array}\right)$

Figure 9: An example of DWI data collection with seven different weightings and gradient directions.

Since $S_{0}$ is the signal when $(|\mathbf{g}|=0)$, the other remaining six diffusion signals $\left(S_{i}, \mathrm{i} \in 1, \ldots, 6\right)$ could be related to $S_{0}$ to form a set of six equations that could be used to obtain the diffusion tensor,

$$
\ln S_{i}=\ln S_{0}-b \hat{\mathbf{g}}_{i}^{T} \mathbf{D} \hat{\mathbf{g}}_{i}
$$

Solving the six obtained equations at each voxel in the DWI data set will result in the final diffusion tensor required. Westin et al. [89] provided a compact analytical solution to equation system (8), removing the necessity to resolve this equation system for every data point. The compact solution provided was developed using dual tensor basis that relies only on the gradient configuration and is 
not dependent on the input data. However, the use of only six diffusion gradient directions will result in an unacceptable low SNR for the resultant DTI derived maps [90]. In particular, as the number of gradient directions used increase, the higher SNR would be obtained. However, a greater number of gradient directions means longer scanning times, which will eventually cause more artifacts due to patient's movements. So an optimum number of gradient directions for data acquisition is needed due to both the SNR requirements and clinical limitations [90].

In the case of carrying out more than seven diffusion measurements with various gradient strengths, gradient orientations, or signal averaging [83], an over constrained system of equations is created, and it can be solved using least squares methods [91,92]. This over-constraining procedure will result in a decrease in the amount of noise produced in the process of calculating diffusion tensors from the diffusion weighted measurements. The resulted over-constrained system $(n \geq 6)$ can be represented in matrix form $(\mathbf{M d}=\mathbf{s})$ :

$$
\left(\begin{array}{cccccc}
x_{1}^{2} & y_{1}{ }^{2} & z_{1}^{2} & x_{1} y_{1} & y_{1} z_{1} & z_{1} x_{1} \\
x_{2}{ }^{2} & y_{2}{ }^{2} & z_{2}^{2} & x_{2} y_{2} & y_{2} z_{2} & z_{2} x_{2} \\
\vdots & \vdots & \vdots & \vdots & \vdots & \vdots \\
x_{n} & y_{n}{ }^{2} & z_{n}{ }^{2} & x_{n} y_{n} & y_{n} z_{n} & z_{n} x_{n}
\end{array}\right)\left(\begin{array}{c}
D_{x x} \\
D_{y y} \\
D_{z z} \\
D_{x y} \\
D_{y z} \\
D_{z y}
\end{array}\right)=\left(\begin{array}{c}
-\frac{1}{b} \ln \frac{S_{1}}{S_{0}} \\
-\frac{1}{b} \ln \frac{S_{2}}{S_{0}} \\
\vdots \\
-\frac{1}{b} \ln \frac{S_{n}}{S_{0}}
\end{array}\right) .
$$

Many algorithms have been developed to estimate the diffusion tensor, and these methods differ considerably in processing speed and the way they deal with data outliers. The linear least square approach (LLS) to solve Equation (9) uses the pseudo-inverse of $\mathbf{M}^{\dagger}$ through a process of singular value decomposition of M [93]:

$$
\mathbf{M}^{\dagger}=\mathbf{V s}^{-1} \mathbf{U}^{T}
$$

where $\mathbf{U}$ and $\mathbf{V}$ are orthogonal matrices, and $\mathbf{s}$ is a diagonal matrix that contains 
the singular values of $\mathbf{M}$. The diffusion tensor $\mathbf{d}$ estimate using least squares is given by

$$
\mathbf{d}=\mathbf{M}^{\dagger} \mathbf{s}
$$

Although the LLS method is widely used in DTI estimation; it may lead to an erroneous estimation because it inaccurately assumes that data outliers are homogenously distributed, and there is still no unified method to define and handle data outliers. Therefore, more advanced estimation methods were introduced to overcome these limitations, such as weighted linear least square (WLLS) and nonlinear least square (NLLS). WLLS method is slower when compared with LLS method, but it will result in the most accurate estimation because it assumes that outliers to be heterogeneously distributed. On the other hand, NLLS method usually results in a more accurate estimation as it minimizes the error in an iterative manner; however, it needs significantly longer processing times, and might become stuck in local minima during the optimization process [94]. The choice of the appropriate DTI estimation method is becoming more important, especially since recent studies have revealed that the quality of WM tracts' reconstruction in poor-quality infant data depend on that choice [94].

\section{Diffusion Tensor Analysis and Measures}

In order to fully characterize anisotropic diffusion or the diffusion ellipsoid, six parameters are needed. These parameters could be obtained by diagonalizing the diffusion tensor $\mathbf{D}$ resulting in a set of three eigenvectors $\left(\hat{v}_{1}, \hat{v}_{2}, \hat{v}_{3}\right)$ which defines the orientation of the axes, and their associated eigenvalues that represent the length of the longest $\left(\lambda_{1}\right)$, middle $\left(\lambda_{2}\right)$, and shortest $\left(\lambda_{3}\right)$ axes. In order for the ellipsoid model to be physically realizable, the obtained eigenvalues of $\mathbf{D}$ must be non-negative. A graphical representation of the ellipsoid model is shown in Figure 10.

In the special case of an isotropic medium, the diffusion ellipsoid takes the 


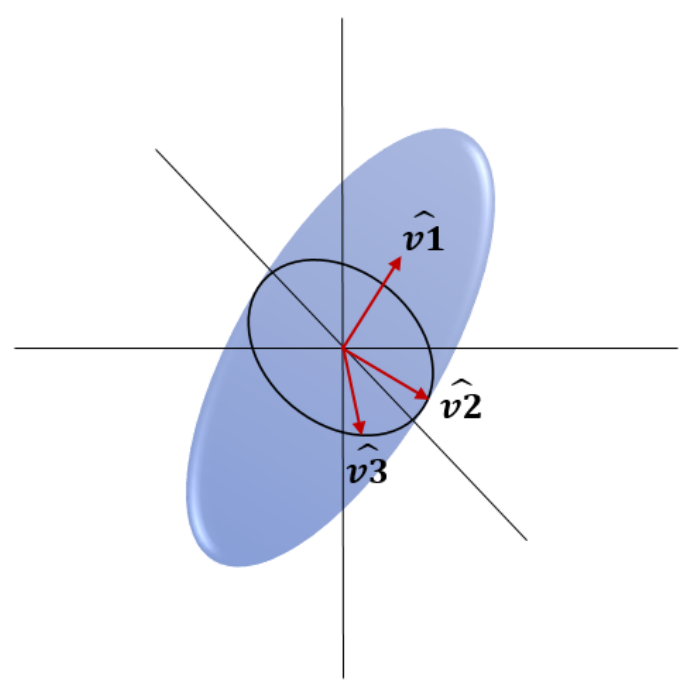

(a) Tensor eigenvectors

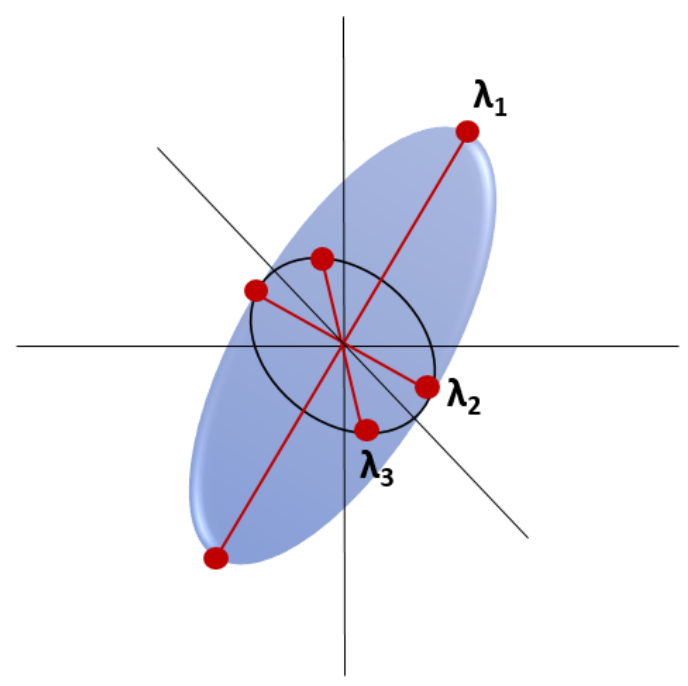

(b) Tensor eigenvalues

Figure 10: Graphical representation of diffusion tensor using the ellipsoid model using three eigenvectors that define the orientation of the ellipsoid, and three eigenvalues that define the principal axes of the ellipsoid.

shape of a sphere because $\lambda_{1}=\lambda_{2}=\lambda_{3}$. While in the case of purely linear anisotropic medium, the diffusion ellipsoid is a line pointing in the $\hat{v}_{1}$ direction as $\lambda_{1}=c$, and $\lambda_{2}=\lambda_{3}=0$. Also, in a planar anisotropic medium, the diffusion ellipsoid converts into an oblate with $\lambda_{1}=\lambda_{2}$, and $\lambda_{1}=0$.

After the diffusion tensor is characterized using the six parameters of the diffusion ellipsoid at each voxel, the next step is to show expressive measurement, and interpretation of 3D image data at each voxel. Therefore, the image information in the eigensystem of $\mathbf{D}$ must be simplified into simpler scalar maps. Next, some of the most commonly used anisotropy and microstructural measurements (see Figure 11) will be listed, namely mean diffusivity (MD), fractional anisotropy (FA), relative anisotropy (RA), axial diffusivity $\left(\lambda_{\|}\right)$, and radial diffusivity $\left(\lambda_{\perp}\right)$. Afterwards, diffusion geometrical measurements (see Figure 12) that are linear $\left(c_{l}\right)$, planar $\left(c_{p}\right)$, and spherical $\left(c_{s}\right)$ measures, and fiber orientation maps (see Figure 13) will be explained. 


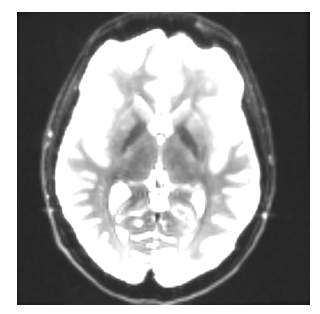

(a) b0

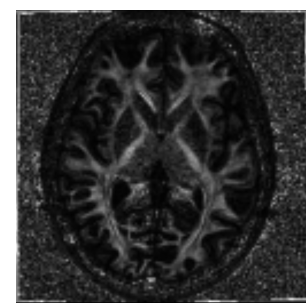

(d) RA

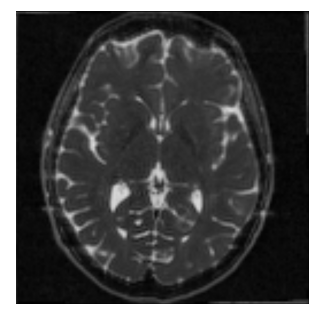

(b) MD

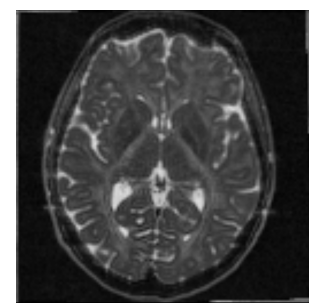

(e) $\lambda_{\|}$

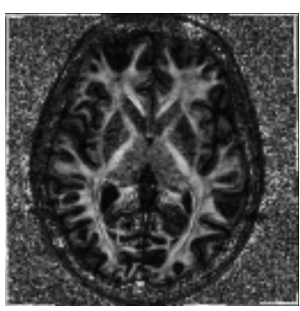

(c) FA

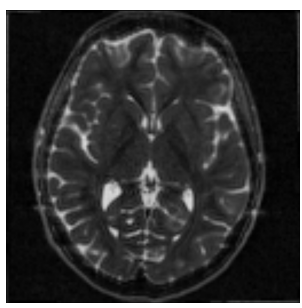

(f) $\lambda_{\perp}$

Figure 11: Various anisotropy and microstructural measurements obtained from DTI human brain. (a) Reference non-diffusion image (b0), (b) mean diffusivity (MD) map, (c) fractional anisotropy (FA), (d) relative anisotropy (RA), (e) axial diffusivity $\left(\lambda_{\|}\right)$, and (f) radial diffusivity $\left(\lambda_{\perp}\right)$.

Mean diffusivity (MD)

In order to get an overall assessment of the diffusion in a voxel or a region, a measurement that is orientation independent must be relied on. The mean diffusivity (MD) (see Figure 11(b)), also called trace, is defined as average of the eigenvalues of $\mathbf{D}$ :

$$
\mathrm{MD}=\frac{\lambda_{1}+\lambda_{2}+\lambda_{3}}{3}
$$

A slightly different definition of the MD has been used to measure the diffusion descent in brain ischemia [95]. Moreover, since the values of MD are higher in CSF than other organized brain tissue, MD is recommended for CSF-related disease studies [96].

Fractional anisotropy (FA)

Many anisotropy measures have been defined, most of which are rotationally invariant. Currently, the fractional anisotropy (FA), which was first described 
by Basser [91], is the most widely used invariant measure of anisotropy. The definition of FA is given by

$$
\mathrm{FA}=\frac{\sqrt{3\left[\left(\lambda_{1}-\mathrm{MD}\right)^{2}+\left(\lambda_{2}-\mathrm{MD}\right)^{2}+\left(\lambda_{3}-\mathrm{MD}\right)^{2}\right]}}{\left.\sqrt{2\left(\lambda_{1}{ }^{2}+\lambda_{2}{ }^{2}+\lambda_{3}{ }^{2}\right.}\right)} .
$$

In the case of physically realizable diffusion that has non-negative eigenvalues, FA ranges from 0 in the extreme case of complete isotropy to 1 for the opposite extreme of linear anisotropy. As illustrated in Figure 11 (c), WM appears whiter since it has higher values of FA. Therefore, reductions in FA are usually used as markers of a change in WM myelination or degradation of WM axonal structure [97].

Relative anisotropy (RA)

Relative anisotropy (RA) is similar to FA, and is defined as the normalized standard deviation of the eigenvalues. RA is also defined as the ratio of the anisotropic part of $\mathbf{D}$ to its isotropic part [98] (see Figure 11 (d)), and is given by

$$
\mathrm{RA}=\frac{\sqrt{\left(\lambda_{1}-\mathrm{MD}\right)^{2}+\left(\lambda_{2}-\mathrm{MD}\right)^{2}+\left(\lambda_{3}-\mathrm{MD}\right)^{2}}}{\sqrt{3 \mathrm{MD}}} .
$$

The values of RA takes the range between 0 (complete isotropy) to $\sqrt{2}$ (complete anisotropy).

Axial and radial diffusivity

In the literature, it was found that combinations of the eigenvalues reveal more particular connections to white matter pathology [80]. The axial or the parallel diffusivity $\left(\lambda_{\|}\right)$measures the diffusion along the principal axis (parallel to axons) (see Figure $11(\mathrm{e})$ ), and is defined as

$$
\lambda_{\|}=\lambda_{1} .
$$


The diffusion in the two minor axes are averaged to produce the radial or the perpendicular diffusivity $\left(\lambda_{\perp}\right)$, which measures the degree of restriction due to memberans and other effects, and is given by

$$
\lambda_{\perp}=\frac{\lambda_{2}+\lambda_{3}}{2} .
$$

These two measurements have been used to observe fiber alterations in several developmental and pathological studies (e.g., Dysmyelinating disorders) [99].

Diffusion geometrical measurements

Based on the generic shape of the diffusion ellipsoid, diffusion could be classified into three types, namely linear, planar, and spherical. The previously described anisotropy measurements (e.g., FA and RA) could not differentiate between these anistropy modes. Westin et al. [89] used the principal eigenvalue for normalization; thus creating the following shape measures, which can detect the linear $\left(c_{l}\right)$, planar $\left(c_{p}\right)$ and spherical $\left(c_{s}\right)$ shapes, respectively (see Figure 12)

$$
c_{l}=\frac{\lambda_{1}-\lambda_{2}}{\lambda_{1}}, c_{p}=\frac{\lambda_{2}-\lambda_{3}}{\lambda_{1}}, c_{s}=\frac{\lambda_{3}}{\lambda_{1}},
$$

where all these measurements have a range from 0 to 1 such that

$$
c_{l}+c_{p}+c_{s}=1 .
$$

Fiber orientation maps

Another parameter that could be extracted from DTI is associated with the fiber orientation maps. The most commonly used method is to show the orientation information through 2D color-coded maps of the main fiber trajectories. This method assumes that the orientation of the fibers is only determined by the eigenvector $\hat{v}_{1}=\left[v_{1 x}, v_{1 y}, v_{1 z}\right]$, which is associated with the largest eigenvalue $\lambda_{1}$. Namely, the absolute value of $v_{1 x}, v_{1 y}$, and $v_{1 z}$ are allocated to the three RGB (red, 


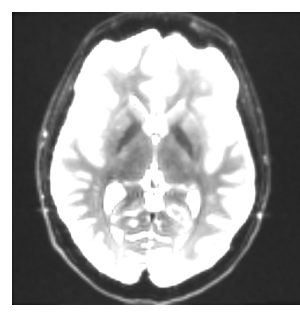

(a) b0

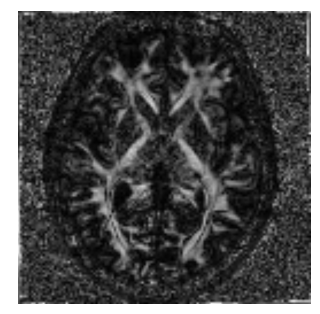

(b) $c_{l}$

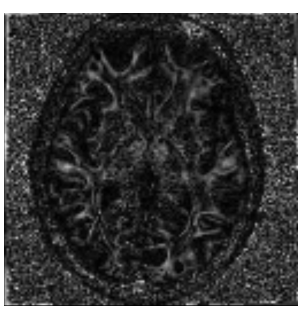

(c) $c_{p}$

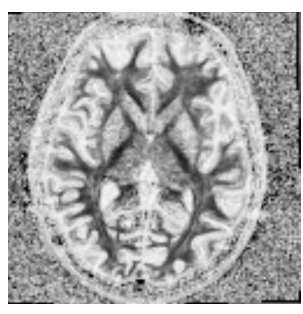

(d) $c_{s}$

Figure 12: Different DTI geometrical-based measurements. (a) Reference nondiffusion image (b0), (b) linear measure $\left(c_{l}\right),(\mathrm{c})$ planar measure $\left(c_{p}\right)$, and (d) spherical measure $\left(c_{s}\right)$.

green and blue) main colors and joined to create one color-coded map [83] (see Figure 13). The information provided by these orientation color maps are unique, and no conventional MRI technique could provide comparable information. Fiber orientations could be used to classify and parcellate specific WM tracts. Therefore, this method could be applied on a wide range of medical applications that necessitate great anatomical details [80].

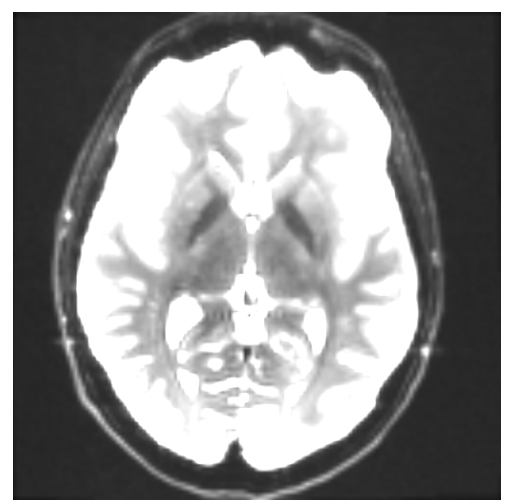

(a) b0

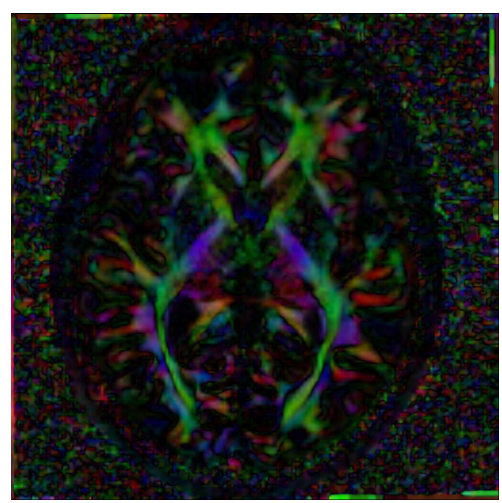

(b) DTI-RGB

Figure 13: Color-coded orientation map. (a) Reference non-diffusion image (b0), and (b) color-coded orientation map (where the colors red, green and blue correspond to diffusion in the $\mathrm{x}, \mathrm{y}, \mathrm{z}$ axes respectively). 


\section{White Matter Fiber Tractography}

2D-based color coded maps could reveal white matter tracts based on voxelby-voxel fiber orientation information; however, they fail to appreciate WM trajectories in 3D space [83]. An alternative way to appreciate WM connection patterns in 3D is using computer-aided 3D tract tracking methods, also called tractography, and is proven to be very valuable in recognizing WM tracts trajectories and their connections with other WM tracts or other GM structures (see Figure 14). In the last decade, many tractography methods $[100,101]$ have been proposed, and these methods could be classified into two categories of tract construction techniques: deterministic approaches that provide only one trajectory for each start voxel, and probabilistic approaches that use energy minimization methods to provide the path with the highest probability to link two selected voxels or regions [83].

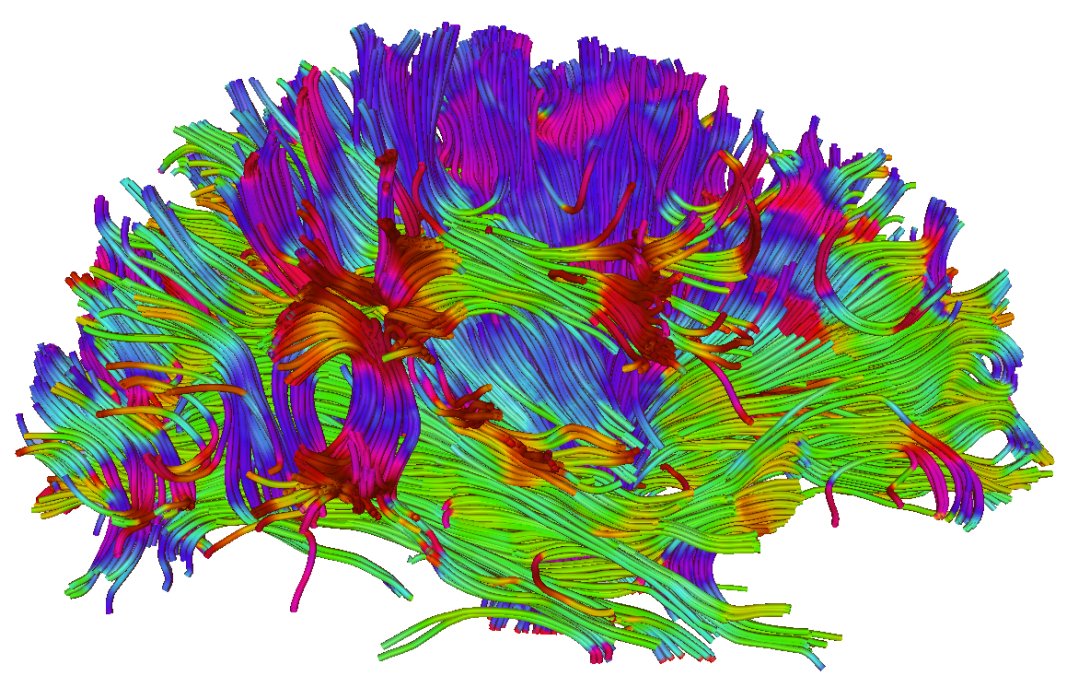

Figure 14: An example of a full brain tractography, where colors have been assigned based on the main direction of the fibers. This tractography was obtained using 3D slicer software [102]. 


\section{Deterministic fiber tractography}

Deterministic (tract propagation) approaches assume that the principal eigenvector $\hat{v}_{1}$ provides a representation to the orientation of the WM fiber tract [101]. There are three steps in the the deterministic models: (i) fiber orientation extraction, (ii) pathway propagation, and (iii) propagation termination [83].

Firstly, local fiber orientation at each voxel have to be estimated. Usually planar diffusion profiles are used as a straightforward estimator; however, this estimate fails if the ellipsoid is isotropic or diffusion profile is planar, since $\hat{v}_{1}$ will be determined based on the noise levels. In these cases, the full diffusion ellipsoid should be used to provide a more reliable estimate.

Secondly, a single pathway have to be propagated based on the full tensor or principal vector information. This process starts from a specified location (seed point), which is usually the center of the image, to form a discrete pathway through the image. However, the obtained discrete pathway does not give a realistic simulation of WM tracts, which are assumed to be adequately smooth. Therefore, sub-voxel estimates of the diffusion tensor are needed to transform the discrete voxel information to a continuous one (see Figure 14). This could be obtained by interpolating the DWI data before diffusion tensor estimation [103], or by interpolating the diffusion tensors directly at suitably selected sub-voxel [101].

Finally, pathway propagation is terminated based on one of two termination criterions most often used. The first is to terminate tracking when the tract enters a low anisotropy region (empirically, FA $>0.15$ is usually used as a threshold to separate WM from GM regions [83]). The second is to terminate tracking when a sharp bend occurs, which means that image resolution is too low to dependably keep tracking [83]. Figure 15 (b) demonstrates an example of the deterministic fibre tractography in the corpus callosum region of interest with 0.2 FA threshold, and 0.7 stopping track curvature. This tractography was obtained by using 3D slicer software [102]. 


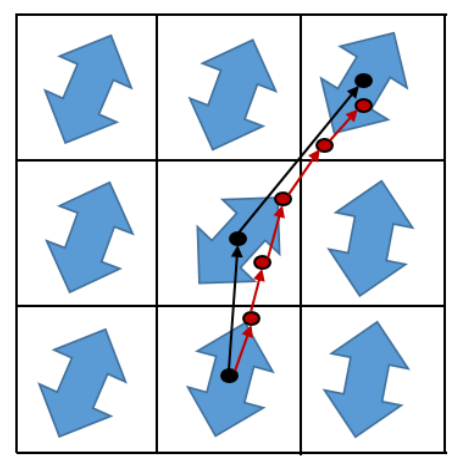

(a) Propagation

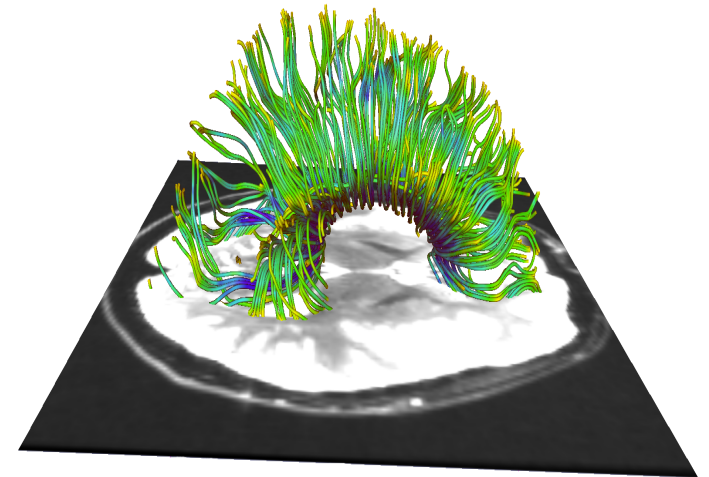

(b) Tractography

Figure 15: Deterministic fiber tractography. (a) Discrete tracking (shown in black) vs. smoothed continuous tracking (shown in red). (b) An example of deterministic fiber tractography. Tracts generated within the corpus callosum region, where colors have been assigned based on the FA values along the tracts. This tractography was generated using 3D slicer software [102].

\section{Probabilistic fiber tractography}

Image noise, patient movement, and other imaging artifacts cause uncertainty in the fiber orientations obtained from deterministic fiber tractography approaches. Therefore, probabilistic fiber tractography approaches were developed to solve this confidence problem [104]. In probabilistic methods, the most probable fiber orientations are estimated as well as a probability distribution of other orientations. Different pathways are traced thousands of times with marginally altered orientations, which allows for assigning a measure of the connection probability. Therefore, fiber connectivity could be assessed between different brain regions using a voxel-based connectivity index [105].

Advantages of probabilistic fiber tractography approaches includes its ability to parcellate full WM tracts. On the other hand, probabilistic methods suffer from a limited accuracy that is restricted by the quality of the information provided by the diffusion tensor as well as the pathway estimated probability distribution function. Another drawback associated with probabilistic methods is their inability to differentiate antegrade from retrograde along the fibers path [104]. 


\section{Clinical Applications}

In neurological studies, the status of each patient should be assessed, and the used imaging modality should be able to clearly discriminate what is abnormal and normal. Conventional MRI lacks sensitivity in distinguishing normal from abnormal on an individual-subject basis [83]. On the other hand, DTI can contribute with new information that conventional MRI could not reveal. The new DTI-based information could be classified into four categories: (i) new contrasts, (ii) white matter morphology, (iii) refined information about anatomical locations, and (iv) connectivity [83]. As illustrated in Section 4, DTI offers many additional image contrasts including the widely used FA anisotropy measurement. The shapes and the size of specific white matter tracts (white matter morphology) could be estimated using DTI [83]. In addition, the superior anatomical information provided by DTI allows for a clearer identification of areas with WM abnormalities [106]. Using 3D fiber reconstruction techniques (e.g., tractography), DTI can provide unique anatomical information about the brain connectivity [101]. This section will overview the clinical applications of DTI in investigating brain abnormalities.

Brain white matter

Although DWI showed some potential in detecting brain WM diseases, DTI measurements provide a better overall discrimination in many brain disorders (e.g., autism) [98]. Examples of these white matter diseases include multiple sclerosis (MS), Alzheimer's disease (AD), cerebral ischemia, Wallerian degeneration, and autism [97]. A brain tumor is a mass or growth of abnormal cells in the brain. Conventional MR with contrast-enhanced T1-weighted and T2-weighted sequences could provide information about the location and extent of these tumors. However, they lack the ability to provide enough information about characteristics and grading of brain tumors [97]. Lower values of FA are usually associated with malignant brain tumors. Fiber tractography could be used to differentiate between 
brain tumor and neighboring healthy WM tracts [83]. As illustrated in Figure 16, DTI is able to reveal structural variations of WM tracts associated with brain tumors.

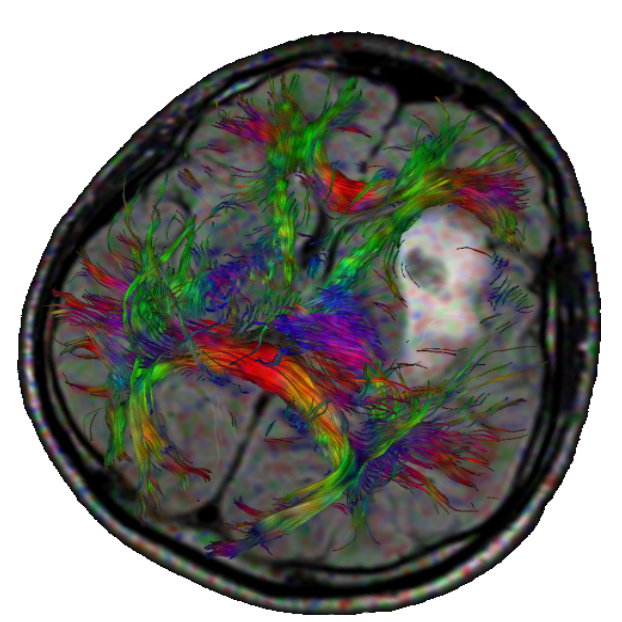

Figure 16: An example of WM structural variations associated to brain tumors. Destruction and deviation of WM tracts caused by anaplastic astrocytoma. Image provided courtesy of [107].

\section{Brain connectivity}

DTI based fiber tractography offers the potential to investigate the neuronal connectivity of the working human brain. Fiber tractography connectivity is of key importance for interpreting both fMRI data and how different parts of the brain are linked together [98]. Many studies combine the structural connectivity information obtained from DTI with other functional connectivity measurements in order to offer more understanding of the connections between brain structure and function [108]. Current DTI tractography techniques suffer from thermal noise and image artifacts. In addition, DTI fiber tractography has to deal with physiological fluctuations such as fiber merging, branching, dividing, etc [98]. Currently, advanced diffusion tensor techniques, such as q ball imaging (Qball) and diffusion spectrum imaging (DSI), are being used to solve these drawbacks. However, these new techniques require very high diffusion $b$ values, and suffer from long 
acquisition times. Their effectiveness is still under investigation, and require more research.

Brain development

The human brain undergoes a continuous development until adolescence, and the most dramatic change is the myelination process, which occurs during the first two years after birth [109]. Conventional MRI techniques may reveal a reduction in brain water content and a rise in WM during the myelination process. However, more information about WM maturation could be available through a DTI-based quantitative analysis [3]. Previous studies indicated an increase in the FA values in some white matter regions [110], and this increase is maintained until adolescence [111]. This new understanding of the normal brain development, enabled researchers to detect any development failures that serve as an early marker of brain injury in newborns [3].

C The Proposed Computer-Aided Diagnosis System

Autism spectrum disorders (ASDs) is a group of lifetime developmental disabilities that are defined by significant social, communication and behavioral challenges. Currently, ASDs denote a significant growing public health concern. According to the report issued by the centers for disease control and prevention (CDC) in 2014, one in 68 children has been diagnosed with ASDs in the United States, which is approximately $30 \%$ greater than previous estimates reported in 2012 of one in 88 children [112]. In addition, this last report indicated that most children with ASDs are currently diagnosed after the age of four, despite the fact that ASDs can be identified as early as age two [112]. Therefore, there is a growing need for developing new techniques for the early diagnosis of ASDs, for an early detection of ASDs could lead to the reduction or the elimination of the manifestation of the disorder through an effective early intervention [4]. 
Recent molecular and functional connectivity studies exploring ASDs confirm the vital importance of investigating localized atypicalities of the brain, and examining the brain distributed neural networks and the connectivity of different areas of the brain [113]. Studies performed at the molecular level have found that minicolumns in the postmortem brains of children with ASD were more reduced, and less compact [114-116]. On the other hand, studies that examined the brain connectivity relied on fMRI to study how various brain areas organize their activities. The results of many of these studies reported that ASD patients show reduced connectivity between frontal and posterior parts during various cognitive tasks as well as during a nontask resting state [117]. Therefore, as concluded from both molecular and functional connectivity studies, brain connectivity and the underlying WM tracts might be impaired in patients with ASDs. Nevertheless, the main drawback of these previous methods is their limited ability to provide sufficient information about the morphology characteristics of these WM tracts and their progress in living human patients [113]. Fortunately, the recent advances in DTI has allowed researchers to study, in a noninvasive manner, both the macrostructure and microstructure (e.g., axons) of WM tracts of the brain. These new advances have allowed the growth of DTI-based studies investigating ASDs in the last decade [113]. These studies used DTI to examine the microstructural properties of WM circuitry and detect abnormalities in WM fiber tract integrity [118]. Currently, there are no medical exams to precisely diagnose ASDs, especially in its early stages. Doctors depend on observation, and talking with parents, physicians and therapists about the child in question to make the diagnosis. Therefore, developing a complete computer aided diagnostic (CAD) system for the early diagnosis of ASDs is a hot point of research.

Since the basic concept of CAD system was proposed in the mid-1980s, there have been a high demand on this new approach to aid neuroradiologists in their heavy workloads. Ever since, many researchers have developed CAD systems for 
various brain diseases [119]. As illustrated in Figure 17, a general CAD system consists of three main steps: ( $i$ ) preprocessing, (ii) feature extraction, and (iii) classification.

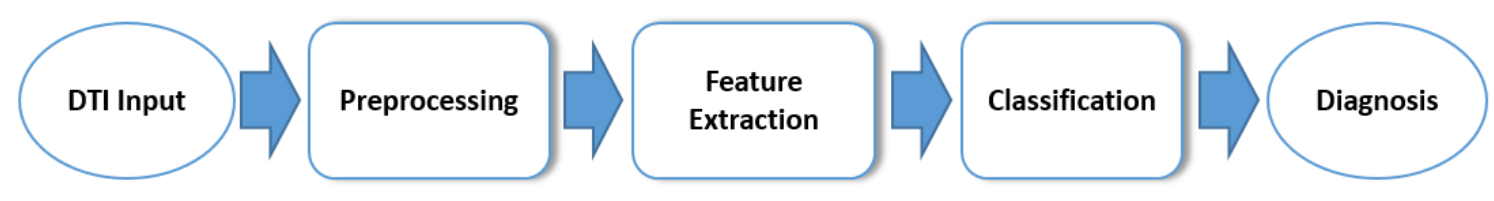

Figure 17: The main steps of a general CAD system.

Preprocessing of input images is crucial for decreasing the complexity and increasing its computation efficiency of CAD systems. Preprocessing includes image normalization, correction, and segmentation. The feature extraction is one of the key steps for CAD systems, and the way the features are extracted changes according to the specific task in hand. Feature extraction scheme usually begin with object enhancement, followed by an initial candidate regions extraction via various segmentation methods, which will be used to generate the final output features. The final step of any CAD system is the classification process, which uses the obtained features as its input to categorize the input data into one of the possible categories (e.g., lesion or normal tissue). Classifiers used in CAD systems usually undergo a teaching process (supervised learning) in which they learn the true category labels for each set of features. These classifiers can be categorized into two types: machine learning-based classifiers such as deep learning, random forest, support vector machine (SVM) and decision tree; and statistical-based classifiers such as Bayesian, k-nearest and artificial neural network (ANN) [119]. Moreover, this final step may involve advanced stages, e.g. identification of brain regions that have significant differences between pathological and control subjects using constructed brain maps.

The ultimate goal of the proposed work in this thesis is to develop a CAD system to classify autistic from control infant brains. This thesis proposes a novel 
DTI-based CAD system for the early diagnosis of ASDs that integrates both shape and connectivity extracted features in the classification process, which is shown in Figure 18. This CAD system consists of three main steps: ( $i$ ) infant brain tissue segmentation from medical images, (ii) extraction of discriminatory features (e.g. shape and connectivity features) for the segmented brain tissues, and (iii) classification of autistic from control infant brains based on analyzing the extracted features of different brain tissues for both control and autistic brains.

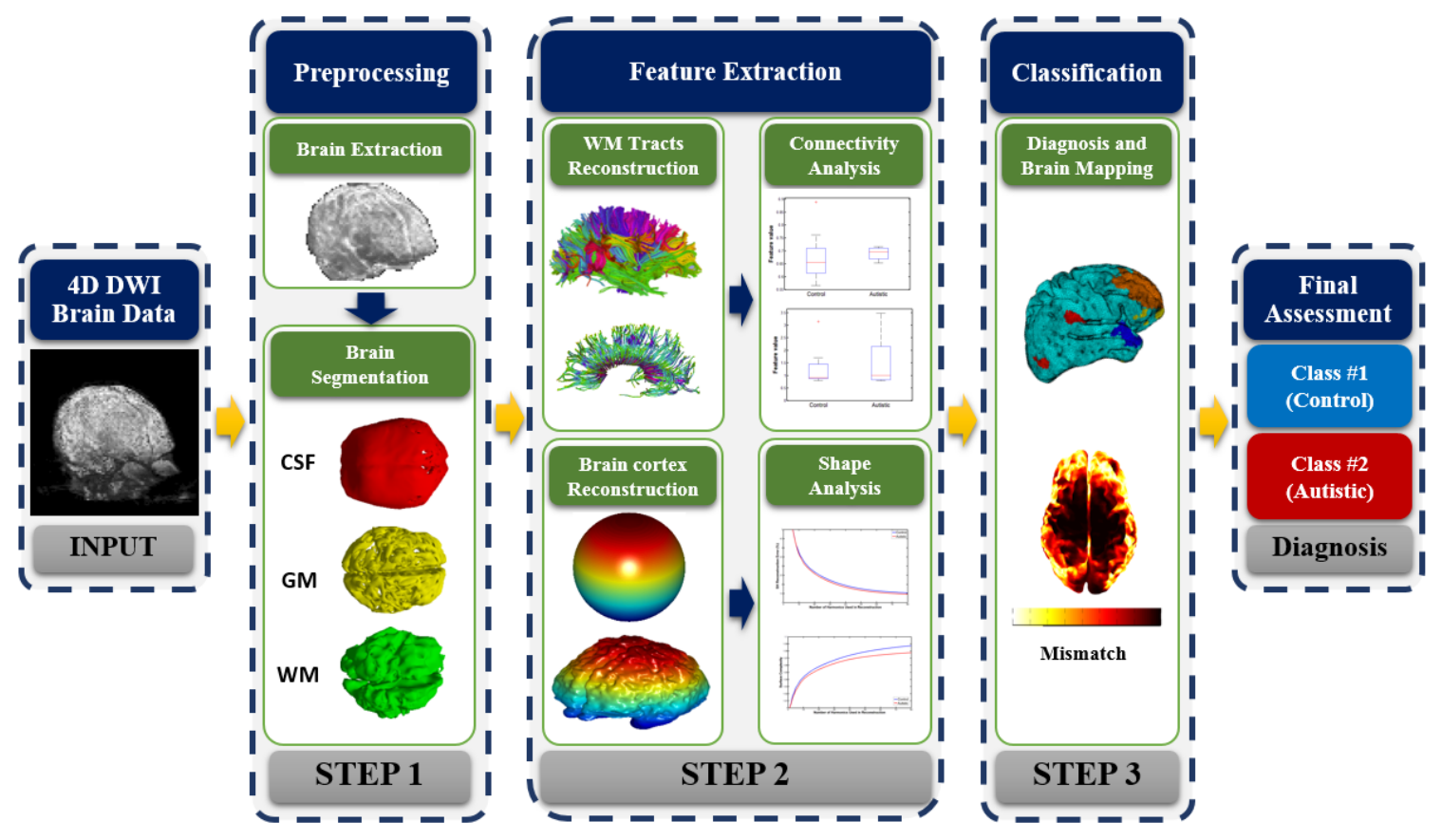

Figure 18: Block diagram of the proposed CAD system for early diagnosis of ASDs.

The input to the CAD system is the medical scans of the infant brain, i.e., $4 \mathrm{D}$ diffusion weighted images. The first step of the proposed CAD system is to accurately segment different brain tissues from the input infant brain data. In this step, the input brain first undergo a quality control procedure to remove scan artifacts, and correct motion and eddy current distortions, followed by brain extraction, in which any non-brain tissues are removed. Then, specific DTI features are extracted, and fused to guide the segmentation process to produce the final segmented brain tissues, i.e., WM, GM, CSF, etc. Following tissue segmentation, the 
next step in the proposed CAD system is to extract discriminatory features, which are numerical values that corresponding to attributes of the segmented structures. In particular, cortex (WM+GM) shape variability is assessed using shape construction methods. In addition to the shape information, WM integrity is further examined through connectivity analysis, where WM tracts are extracted and analyzed using various DTI connectivity measurements. Finally, both the extracted shape and connectivity features will serve as an input for the system classifier. The used classifier will correlate between both connectivity and shape findings to provide better discrimination between autistic and control infant brains.

\section{Thesis Organization}

This thesis is divided into four chapters. The following remarks summarize the scope of each chapter:

- Chapter (I) briefly reviews the main classes of medical imaging modalities; provides the necessary principles of DTI, such as the diffusion tensor analysis and applications; and introduces the basic contributions of the proposed CAD system for early diagnosis of ASDs from infant diffusion tensor images.

- Chapter (II) presents a novel adaptive atlas-based framework for the automated segmentation of different brain structures from infant diffusion tensor images. The proposed framework provides a more accurate segmentation of different infant brain structures in the isointense age stage (6-12 months) by integrating superior DTI image features in the segmentation procedure. The proposed segmentation framework relies on a joint Markov-Gibbs random field (MGRF) model that accounts for three image descriptors: (i) a nonnegative matrix factorization (NMF) based visual appearance to describe the empirical distribution of NMF fused DTI features, (ii) a shape model that is adapted during the segmentation process guided by the first- and second- 
order visual appearance characteristics of the new NMF-fused image features, and (iii) a 3D spatially invariant second-order MGRF homogeneity descriptor to account for the large inhomogeneity existing in infant brains.

- Chapter (III) presents the feature extraction and the brain classification steps of the proposed CAD system. The proposed CAD system relies on both shape and connectivity features to accurately differentiate between control and autistic brains. The proposed shape analysis detects brain cortex variability using a spherical harmonic (SH) analysis, and brain shape complexity is described with two shape measurements, namely, $\mathrm{SH}$ reconstruction error and surface complexity. The proposed connectivity analysis relies on tractography methods to reconstruct WM tracts of the segmented brains. Three different DTI measurements, namely, fractional anisotropy (FA), axial diffusivity $\left(\lambda_{\|}\right)$, and radial diffusivity $\left(\lambda_{\perp}\right)$ are generated and mapped to the extracted WM tracts to perform statistical analysis to detect differences between autistic and control brains. K-means classifier were used for each of the extracted features to evaluate their diagnostic capabilities.

- Chapter (IV) presents a general discussion about the presented research and its results, followed by the main conclusions and the possible areas for future work. 


\section{CHAPTER II}

\section{INFANT BRAIN SEGMENTATION}

Accurate and reliable segmentation of anatomical structures plays a vital role in any computer-aided diagnostic (CAD) system for brain disorders. Moreover, infant brain segmentation is of great importance in developing CAD systems capable of revealing patterns of early brain development. Unfortunately, MR infant brain segmentation is particularly challanging compared with adult brain segmentation. This chapter introduces a novel adaptive atlas-based framework for the automated segmentation of different brain structures from infant diffusion tensor imaging (DTI). The proposed framework provides a more accurate segmentation of different infant brain structures in the isointense age stage (6-12 months) by integrating superior DTI image features (e.g., fractional anisotropy (FA)) in the segmentation procedure. To model the brain images and their desired region maps, a joint Markov-Gibbs random field (MGRF) model is proposed. The joint MGRF model accounts for three image descriptors: (i) a nonnegative matrix factorization (NMF) based visual appearance to describe the empirical distribution of NMF fused DTI features, (ii) an adaptive shape model, and (iii) a 3D spatially invariant second-order MGRF homogeneity descriptor. The NMF-based visual appearance descriptor is modeled using a $K$-means classifier with centroids calculated in the training phase. The main advantage of NMF feature fusion is its ability to extract meaningful features from the large dimensional DTI feature space; thus, creating a new feature space, in which dimensionality was reduced and different classes were better separated. Unlike most of the existing techniques that depend mainly 
on predefined shape prior probabilities, the proposed framework involves the use of an adaptive shape prior. The proposed adaptive shape model is constructed from a prior atlas database built using a subset of co-aligned training data sets that is adapted during the segmentation process guided by the first- and second-order visual appearance characteristics of the new NMF-fused image features. These characteristics are described using voxel-wise image intensities and their spatial interaction features. In order to perform a more accurate segmentation, a secondorder MGRF spatial interaction model is added to account for the large inhomogeneity existing in infant brains. The high accuracy of the proposed segmentation approach was confirmed by testing it on 9 in-vivo infant DTI brain data sets using three metrics: the Dice coefficient, the 95-percentile modified Hausdorff distance, and the absolute volume difference.

\section{A Introduction}

Segmentation of anatomical structures such as white matter (WM), gray matter (GM), and cerebrospinal fluid (CSF) regions is a crucial step in any CAD system for brain disorders [119]. In particular, segmenting an infant brain MR image contributes much into the analysis and treatment of brain injury and disorder resulting from infant brain prematurity [120]. Although manual segmentation performed by experts remains the gold standard, automated and semi-automated segmentation approaches are necessary due to the time consuming nature and performance variability of manual segmentation procedures. Brain MRI segmentation meets with many challenges that stem from image noise, inhomogeneity, artifacts (e.g., partial volume effect), and boundary discontinuities due to the similarity in the visual appearance of adjacent brain structures. Moreover, the diffusionsensitizing gradient used in diffusion weighted imaging (DWI) yields an amplification effect to the distortions that are related to patient motion [121]. This chapter targets infant brain DTI segmentation that is more difficult than adult brain seg- 
mentation, which can be conducted using only the image intensity. Infant brains have a greatly reduced contrast, including a reverse in contrast in the WM and GM because of the immaturity of the brain tissues [122], and a higher amount of noise [123]. Eddy current artifacts, and bulk motion distortions are additional difficulties that exist, especially in unsedated infants (see Figure 19).

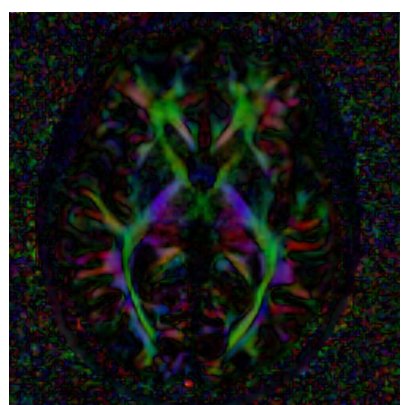

(a) Adult

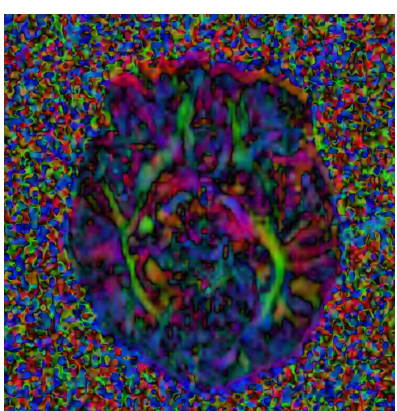

(b) Infant

Figure 19: Comparison between infant and adult brains based on color coded orientation maps generated from DTI

For infant brains, most of the WM is still unmyelinated and its water content is close to that of the GM, resulting in the lower contrast between the tissue classes. Moreover, both the WM and GM have the same intensity at about nine months of age [124]; hence, it is difficult to classify the brain tissues using only the intensity. Additionally, major partial volume effects occur due to the contrast inversion between the WM and GM comparing to the MR adult brain images. The unmyelinated WM intensity is just between intensities for the GM and CSF tissues, so that partial volume averaging often misclassifies the average between the latter two tissues as the unmyelinated WM [122]. Furthermore, the acquisition factors, such as long scan duration, small voxel size, and low signal-to-noise ratio (SNR), also hinder infant brain imaging. High-resolution images are essential to show the infant brain structure because of its much smaller size compared to the adult brains [120]. However, small-size voxels lead to noisy infant scans with reduced contrast, i.e. with low contrast-to-noise ratio (CNR). To improve the CNR, the scan duration has to be increased; but, it is too risky for an infant to be anesthetized for 
a long period. As it is important to minimize scan times, usually infants are fed and then wrapped to prevent movement [125]. New imaging technologies such as parallel imaging or next-generation multi-channel imaging coils can decrease the scan time and increase the CNR and SNR.

A large variety of segmentation techniques have been developed, in the last two decades, in order to address the brain MRI segmentation challenges. The next section will provide an overview of the related current infant brain segmentation techniques.

\section{B Related Work}

Modern techniques for brain MRI segmentation can be roughly categorized into three main groups: ( ) probabilistic or statistical methods, (ii) atlas-based methods, and (iii) deformable model techniques. Most techniques primarily deal with adult brain segmentation, and few techniques are suited for infant brain segmentation. The following subsections will overview and address the limitations of the current segmentation techniques that deal with MR infant brains, with a special focus on those who incorporate DTI measurements in their framework.

\section{Probabilistic Segmentation}

These algorithms involve prior models that describe signal distributions of each brain structure. Xue et al. [122] employed a parametric Gaussian density estimation with an Expectation-Maximization (EM) algorithm and constrained spatial homogeneity of the MR images with a Markov random field (MRF) prior. Partial volume averaging effects were eliminated by predicting misclassification (e.g., of an "averaged" CSF and GM into an intensity similar to a WM). Automated segmentation of brain structures, such as WM, CSF, central GM, and cortical GM by Anbeek et al. [126] used probability maps to segment each brain tissue class with a $K$-nearest neighbor classifier. Anbeek employed features such as voxel intensities 
and coordinates that were manually constructed. Cardoso et al. [127] proposed a posteriori EM multi-modality segmentation algorithm, which included the use of a relaxation approach that iteratively adjusts the tissue priors to match the input subject. The segmentation algorithm also included intensity non-uniformity (INU) correction model, an MRF-based homogeneity descriptor, and a correction of partial volume containing voxels. More generalized image priors were introduced by Gui et al. [128], where the creation of these priors was based on a fusion between different high-level brain morphology information, namely tissue connectivity, structure and relative positions. This morphology information was incorporated in the segmentation process through a neighbourhood selection criteria, which guides a region growing algorithm in separating WM, GM, and CSF. Patrial volume errors are corrected by imposing a condition on WM growing into neighbouring extra-ventricular CSF. The proposed segmentation approach integrated the high level morphological information in other segmentation techniques, such as the marker-based watershed segmentation [129] and region based active contours [130]. Wang et al. [131] segmented T1, T2 and diffusion-weighted brain images using a sparse representation of the complementary tissue distribution. Initially, the brain tissue was segmented into different structures using a patch-based technique with a library of multi-modality images, having been aligned with their ground-truth segmentation maps. Then the segmentation was refined by integrating geometric constraints.

Statistical-based techniques are fast to implement compared to other segmentation methods; however, they depend only on predefined probability models that cannot fit all of the possible real data distributions. This is because actual intensity distributions of brain structures are greatly affected by several factors, such as the unique patient and scanning parameters. Also due to the similar intensities for the different brain tissue structures of the infant MR brain images, segmentation techniques only based on the intensity remain inaccurate. 


\section{Atlas-Based Segmentation}

Atlas-based approaches have emerged as a powerful segmentation tool. These approaches are based on a priori knowledge about the location of different brain structures, and treat the segmentation problem as a registration task. Prastawa et al. [132] developed an atlas-based segmentation algorithm for newborn brain MRI, which uses a registered probabilistic brain atlas. In order to create this atlas, three segmentations were generated by the $K$-nearest neighbor segmentation with different manually selected samples for each tissue class. Then, these segmentations were refined manually, registered using affine transformation, and then averaged to create the initial atlas. To create the final prior probabilities, population variability was simulated by applying an additional blurring to the average segmentations. The proposed segmentation started with a robust graph clustering and parameter estimation to model the initial intensity distributions, then bias correction is performed using the initial intensity distributions as well as the spatial priors. The corrected initial segmentation was refined using nonparametric kernel density estimates to produce the final segmentation. To eliminate bias and variability associated with manual interaction, Weisenfeld et al. [120] proposed a fully automated, adaptive classifier fusion algorithm for segmenting myelinated WM, unmyelinated WM, CSF, cortical GM and subcortical GM from brain MRI of newborn infants. Tissue class intensity distributions were estimated nonparametrically using a set of samples of representative tissue MR intensity values (prototypes) for each tissue class. The proposed algorithm started with a library of template MRI images, and each template has a large number of manually selected tissue class prototypes. After template-subject registration, different prototype lists lead to nonparametric density estimates that result in different segmentations. These segmentations are then fused to increase the final segmentation accuracy. To overcome the problem of structural differences between the templates and the study subject, the prototype lists were iteratively refined (until convergence) to produce new seg- 
mentations, which leads to a new fused classification. In the case of tissue classes with the same intensity (e.g., cortical and subcortical GM), they used an atlas of spatially varying prior probabilities. For the construction of this atlas, they used fifteen subjects that were segmented using a semi-automated segmentation algorithm [133]. They were aligned using affine transformations, and each set of voxel probabilities was calculated based on the relative frequency of each class label. Shi et al. [134] proposed a longitudinal neonatal brain image segmentation framework that includes a bias correction step as well as the use of a subject-specific tissue probabilistic atlas, and a longitudinal atlas that was built from late time scans of the same subject. The use of a subject-specific atlas will lead to a smaller anatomical variability, which will eventually lead to a better segmentation. To construct a subject-specific atlas, the late time-point scans (one-year-old and two-year-old) were segmented using an adaptive fuzzy segmentation algorithm (AFCM) to get the probabilistic maps of CSF, GM, and WM tissue classes. After atlas reconstruction, an iterative joint registration-segmentation process was performed to align the constructed atlas and to get the atlas-based tissue segmentation of the neonatal images. The registration method used was based on the HAMMER nonlinear registration algorithm [135], which gradually increased the degree of nonlinear warping as the segmentation became better. To compensate for local intensity inhomogeneity existing in infant brains, Kim et al. [136] proposed the use of spatial intensity growth maps (IGM) for T1 and T2 weighted images. The IGM, which was calculated based on a voxel-wise linear regression model, captured intensity changes of $20-25 \%$ in immature WM regions between one and two-year-old scans. The IGM modified images were then segmented using an enhanced EM segmentation method. In order to create the optimal tissue priors, they initially mapped an existing four-year-old atlas [137] to the one-year-old atlas [138] using deformable registration. Then, the initial priors was improved by using expert manual segmentations of additional datasets of the one-year-old scans. The obtained prior 
probability maps were updated with fourteen training subjects, which were segmented using the proposed segmentation algorithm but with the updated priors. This update procedure was iteratively performed, based on the resulting difference maps to the ground truth for each tissue class, and was terminated when the prior maps displayed less than $1 \%$ accumulative variation over all priors. Wolff et al. [118], Elison et al. [139], and Neda et al. [140] introduced an atlas building procedure that contains two different registration frameworks using DTI and T2weighted images. The intra-subject and inter-modality registrations were based on a multi-scale approach that employs both affine and B-spline transformations, using the normalized mutual information (MI) as a matching metric. Scans taken at different time intervals are linearly mapped to an atlas constructed from a patient at the age of one year. They are subsequently mapped using a nonlinear transformation to a T2-weighted atlas, and tensor images are estimated from the aligned DWI and averaged using the log-Euclidean method to produce a final DTI atlas.

Atlas-based segmentation techniques show more accuracy with respect to statistical-based techniques. Nevertheless, they are still challenged by atlas selection, combination, and the associated heavy computation time. Moreover, the obtained segmentation accuracy is highly dependable on the involved registration quality. Another major drawback of atlas-based segmentation algorithms is their dependency on the selected features that will be used to link between the test subject and the prior (training) data used in the construction of the atlas. For example most of the current techniques use signal intensity to find the correspondence between the data to be segmented and the prior atlas. This may lead to inaccurate segmentation results as signal intensities vary due to many factors, such as the age, patient, and scanner. 


\section{Deformable Models-Based Segmentation}

In order to obtain continuous segmentation of brain structures, deformable boundaries have also been recognized as accurate segmentation techniques of MR brain tissues. Deformable models-based segmentation techniques are based on deforming curves or surfaces (2D image domain) or hyper-surfaces (higher dimensional image data) under the influence of both internal and external forces to delineate the targeted object boundary. Leroy et al. [141] introduced a surface reconstruction framework for automated segmentation of the cortical interface between WM and GM in MR infant brains. After spatial intensity inhomogeneity correction, the initial cortical surface was identified based on two local priors that are not affected by the intensity inhomogeneity. The first prior was the relatively steady thickness and darker intensity of the cortical ribbon, which was detected using the morphological top hats method [142]. The second prior was the ridge segments of WM intensity, which was detected by comparing the mean curvature of iso-intensity surfaces [143]. The final reconstruction of the inner cortical surface was obtained by applying deformation on two surfaces located at each side of the cortical region, and the competition between these two surfaces results in a more robust reconstruction. The deformation method used [144] was initialized on each side of the inner cortical surface, and the surfaces' evolution was controlled by a speed function that accounts for both feature intensity and neighbourhood configuration. A level set segmentation framework was developed by Wang et al. [145], in which a combination of local intensity information (modeled by the local Gaussian distribution fitting (LGDF) [146]), atlas spatial prior, and a constraint on the thickness of the cortical surface was used to construct the final coupled level sets. An initial segmentation was first obtained by using convex optimization for the coupled level sets based on global image statistical information and atlas spatial prior, and then it was used as an initialization for the coupled level sets. This random initialization provides a more robust segmentation results. In 
another study by the same author, Wang et al. [147] proposed a longitudinally guided level-sets segmentation method. The proposed segmentation framework takes advantage of the fact that the major sulci and gyri, which are established in adult brains, are already present in infants. Therefore, they use the longitudinal segmentation result from the late-time-point scans to guide the segmentation of neonatal brains. The segmentation of the late-time-point scans can be attained with good accuracy by many of the well-established segmentation methods. In particular, two-year-old late-time-point and neonatal brain images are independently segmented using the adaptive fuzzy c-means algorithm [148] and the previously described coupled level sets segmentation framework [145]. Then, the neonatal segmentation was refined by wrapping the older two year scan to the neonatal space using the HAMMER deformable registration [135]. A 4D multi-modality extension to the last segmentation framework is presented by Wang et al. [149]. They extended the previous work to be able to segment six-month-old images through integrating both one-year and neonatal scans to guide the desired segmentation. To overcome the intensity issues associated with six-month scans, they incorporated the use of fractional anisotropy (FA) maps measured from DTI. Moreover, temporally consistent segmentation results were obtained by a longitudinally consistent term. Recently, Wang et al. [150] proposed a patch-driven level set method for segmenting neonatal brain MR images using a sparse representation technique, which effectively creates a subject-specific atlas from a library of aligned, manually segmented images. The resultant tissue probability maps are then refined by measuring the similarities between the current patch and its neighboring patches. The final segmentation was obtained by integrating the refined probabilities into a coupled level set framework.

The main advantage of deformable model-based segmentation techniques is their ability to segment connected (non-scattered) objects more accurately than the other segmentation methods. However, the accuracy of this method is based 
on the accurate design of the guiding forces (statistical, geometric, etc.) in addition to the initialization of the model.

\section{The Proposed Segmentation Framework}

In addition to the limitations associated with each segmentation category, most of the previously described MR infant segmentation techniques were dedicated to segment infant brains either in the early infantile stage ( $\leq 5$ months) or early adult-like stage ( $\geq 12$ months) by using a T1 or T2 scan or the combination of both $[120,122,127,128,132,134,136,141,145,147]$. However, these methods would fail in the case of infants in the isointense stage (6-12 months), which is the primary focus of this thesis, because both WM and GM have roughly the same intensity levels (see Figure 20). Therefore, a segmentation framework that integrates other image contrasts (e.g., FA maps [131,149]) would result in a better differentiation between WM and GM in the isointense stage. However, neither of those studies tried to integrate other DTI image contrasts in their segmentation procedure. In addition, current DTI-based infant brain segmentation techniques suffer from the following limitations: (i) atlases constructed from multiple modalities (e.g., T2-weighted and DTI) will decrease the segmentation accuracy as a result of dissimilar contrast levels and inter-slice variability, (ii) using nonlinear registration negatively affects the shape information, thus it prevents carrying out any shape-based statistical analysis on the segmented data, which could be beneficial in inspecting the relationship between WM and GM morphology [151], and (iii) many of them rely on longitudinal information, which is not available in most cases.

To overcome the limitations mentioned above, this thesis proposes a novel adaptive atlas-based framework for the automated segmentation of different brain structures from infant DTI images (see Figure 21). By integrating superior DTI image features (e.g., FA) in the proposed framework, different infant brain struc- 


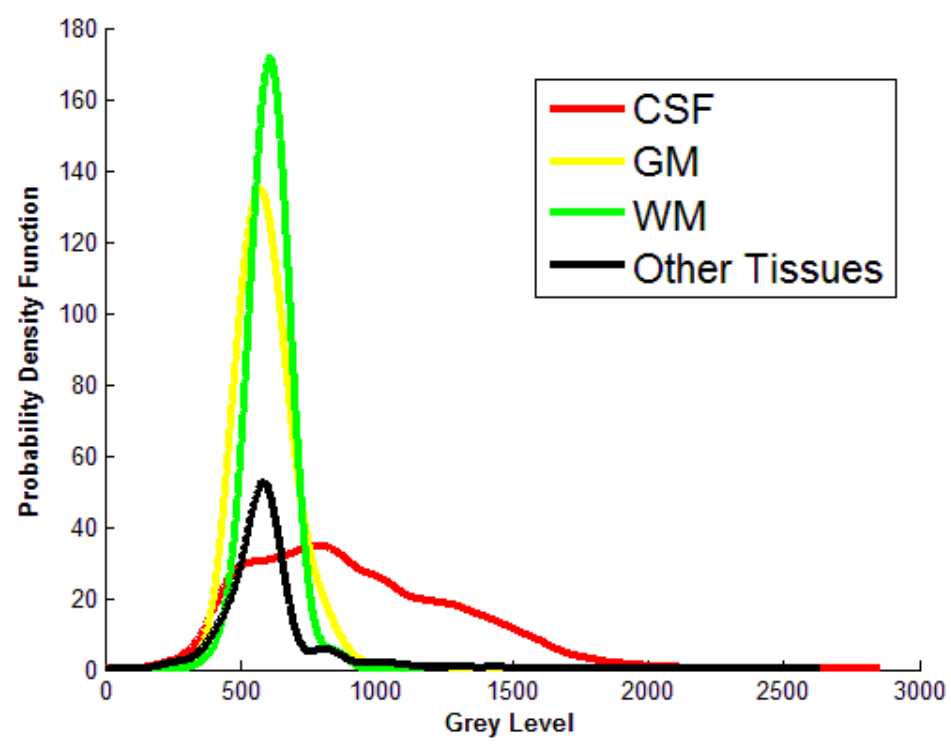

Figure 20: The intensity histogram of different brain tissue classes (i.e., CSF, GM, $\mathrm{WM}$, and other brain tissues) using non-diffusion b0 (T2-weighted) scan of an infant in the isointense stage (6-12 months). As seen from the resultant histogram, a high overlap between WM and GM intensities occurs in the isointense stage.

tures can be separated more accurately, especially in the isointense stage. A novel joint MGRF segmentation model is introduced to model the brain images and their desired region maps. In particular, the joint MGRF model relies on three image descriptors: $(i)$ an NMF-based visual appearance to describe the empirical distribution of NMF fused DTI features, (ii) an adaptive shape model, and (iii) a 3D spatially invariant second-order MGRF homogeneity descriptor. This segmentation framework utilizes an NMF features fusion of the large dimensional DTI feature space to extract new meaningful features with reduced dimensionality and increased separation ability. The NMF-based visual appearance descriptor is modeled using a $K$-means classifier with centroids predefined in the training phase. To overcome the atlas bias problem related with most existing techniques, the proposed framework involves the use of an adaptive shape prior. The proposed adap- 
tive shape model is constructed from a prior atlas database built using a subset of co-aligned training data sets that is adapted during the segmentation process guided by the first- and second-order visual appearance characteristics of the new NMF-fused image features. These characteristics are described using voxel-wise image intensities and their spatial interaction features. Finally, a second-order MGRF spatial interaction model is added to account for the large inhomogeneity existing in infant brains. Details of the proposed segmentation framework will be presented in the following sections.
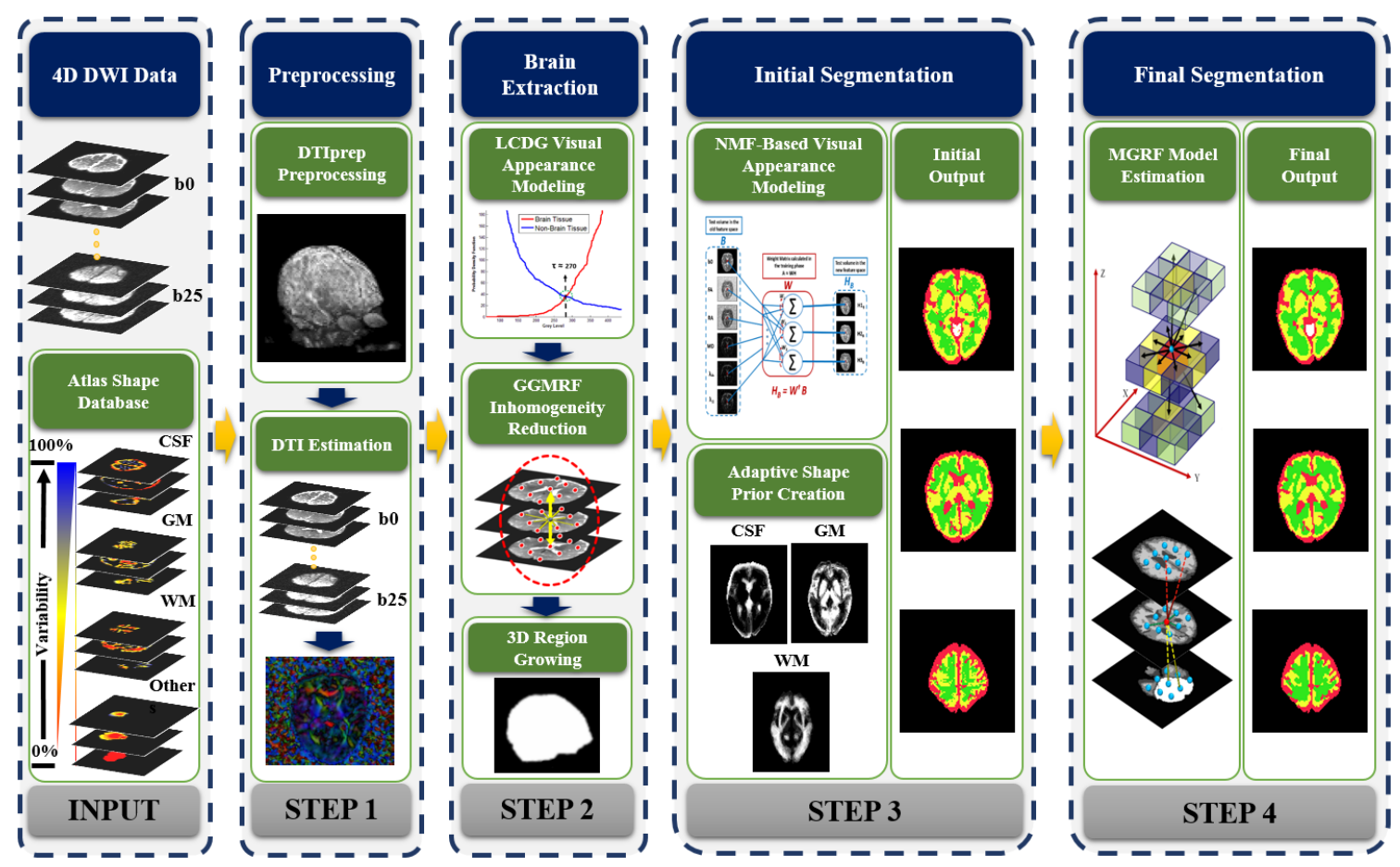

Figure 21: The basic steps of the proposed framework for segmenting different structures from infant DTI brain data. 
Before describing the proposed methods, the basic notation used throughout this chapter will be presented,

- $\mathbf{R}=\{(x, y, z): 0 \leq x \leq X-1,0 \leq y \leq Y-1,0 \leq z \leq Z-1\}-$ a finite arithmetic lattice supporting digital images and their region maps, and a voxel $s=(x, y, z)$ is associated with its neighbors, $\{(x+\xi, y+\eta, z+\zeta)$ : $\left.(x+\xi, y+\eta, z+\zeta) \in \mathbf{R} ;(\xi, \eta, \zeta) \in \nu_{s}\right\}$ where $\nu_{s}$ is the 26-neighbourhood defined by $\xi \in\{-1,0,1\}, \eta \in\{-1,0,1\}$, and $\zeta \in\{-1,0,1\}$.

- $\mathbf{g}=\left\{g_{x, y, z}:(x, y, z) \in \mathbf{R} ; g_{x, y, z} \in \mathbf{Q}\right\}$ - a gray scale image taking values from a finite set $\mathbf{Q}=\{0,1, \ldots, Q-1\}$.

- $\mathbf{m}=\left\{m_{x, y, z}:(x, y, z) \in \mathbf{R} ; m_{x, y, z} \in \mathbf{L}\right\}-$ a region map taking values from from a finite set $\mathbf{L}=\{0, \ldots, L\}$.

- $\mathbf{A}=\left\{a_{i, n}: i=1, \ldots, I, n=1, \ldots, X Y Z ; a_{i, n} \in \mathbf{Q}\right\}-$ a 2D matrix contains image features of all $n$ voxels in a vector form, where $i$ is the dimension size of image features.

- $\mathbf{W}=\left\{w_{i, j}: i=1, \ldots, I, j=1, \ldots, J ; w_{i, j} \in \mathbb{R}^{+}\right\}$- a 2D matrix contains $J$ basis image features.

- $\mathbf{H}=\left\{h_{j, n}: j=1, \ldots, J, n=1, \ldots, X Y Z ; h_{i, j} \in \mathbb{R}^{+}\right\}$- a 2D matrix contains $n$ voxels in new feature space of size $J$.

C Methods

The proposed segmentation framework is divided into three main components: (i) preprocessing, (ii) brain extraction, and (iii) the joint MGRF segmentation model. The details of each of these components will be provided in the following subsections. 


\section{Preprocessing}

\section{DWI Image Preprocessing}

Compared to conventional MRI, DWI is more vulnerable to image noise and artifacts (e.g. eddy-current and venetian blind artifacts). Moreover, DWI has relatively longer acquisition times, which cause more motion artifacts, especially in unsedated infants, in addition to slice-wise and gradient-wise inconsistencies. Also, during DWI acquisition, fast gradient switching existing in echo-planar imaging (EPI) will yield an amplification effect to various image distortions. Therefore, DWI screening is an essential step in any DTI-based study to eliminate any bias that may exist with data collection, which potentially could affect the final outcome of the study [152]. Some examples of DWI intensity artifacts are shown in Figure 22.

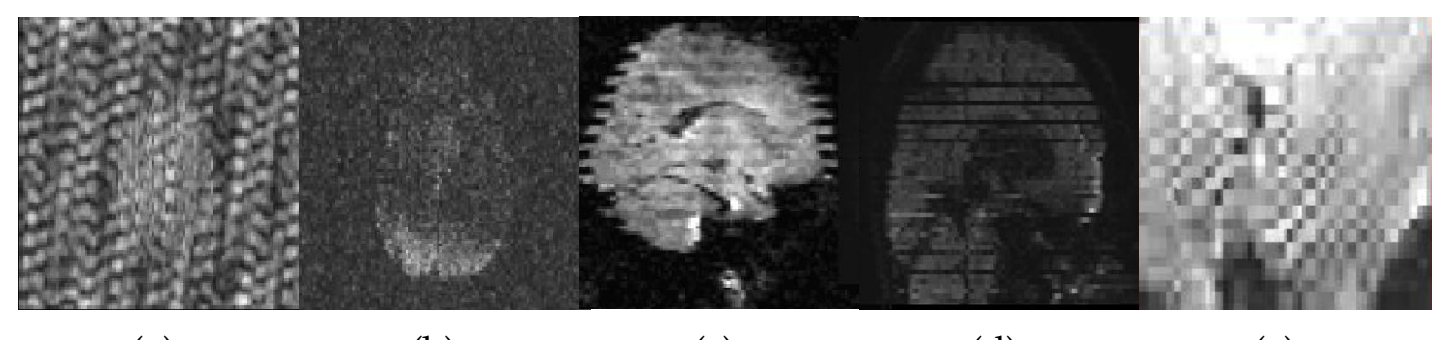

(a)

(b)

(c)

(d)

(e)

Figure 22: Examples of DWI acquisition artifacts: (a) electromagnetic interference, (b) extreme signal loss, (c) venetian blind, (d) slice-wise inconsistencies, and (e) checkerboard artifacts. Courtesy of [152].

In order to perform the required DWI quality control (QC), DTIprep software [152] was used, which automatically detects and removes various scan artifacts, and correct motion and eddy current distortions. Using the DTIprep generated comprehensive report and the obtained results, scans with strong remaining artifacts were eliminated. In addition, resultant data sets with low number of gradient directions, which will produce DTI estimates with low SNR, were excluded from any further processing. The main steps involved in the QC procedure per- 
formed by DTIprep software are summarized in Algorithm 1 [152], and the complete DTIprep software details could be found in [152].

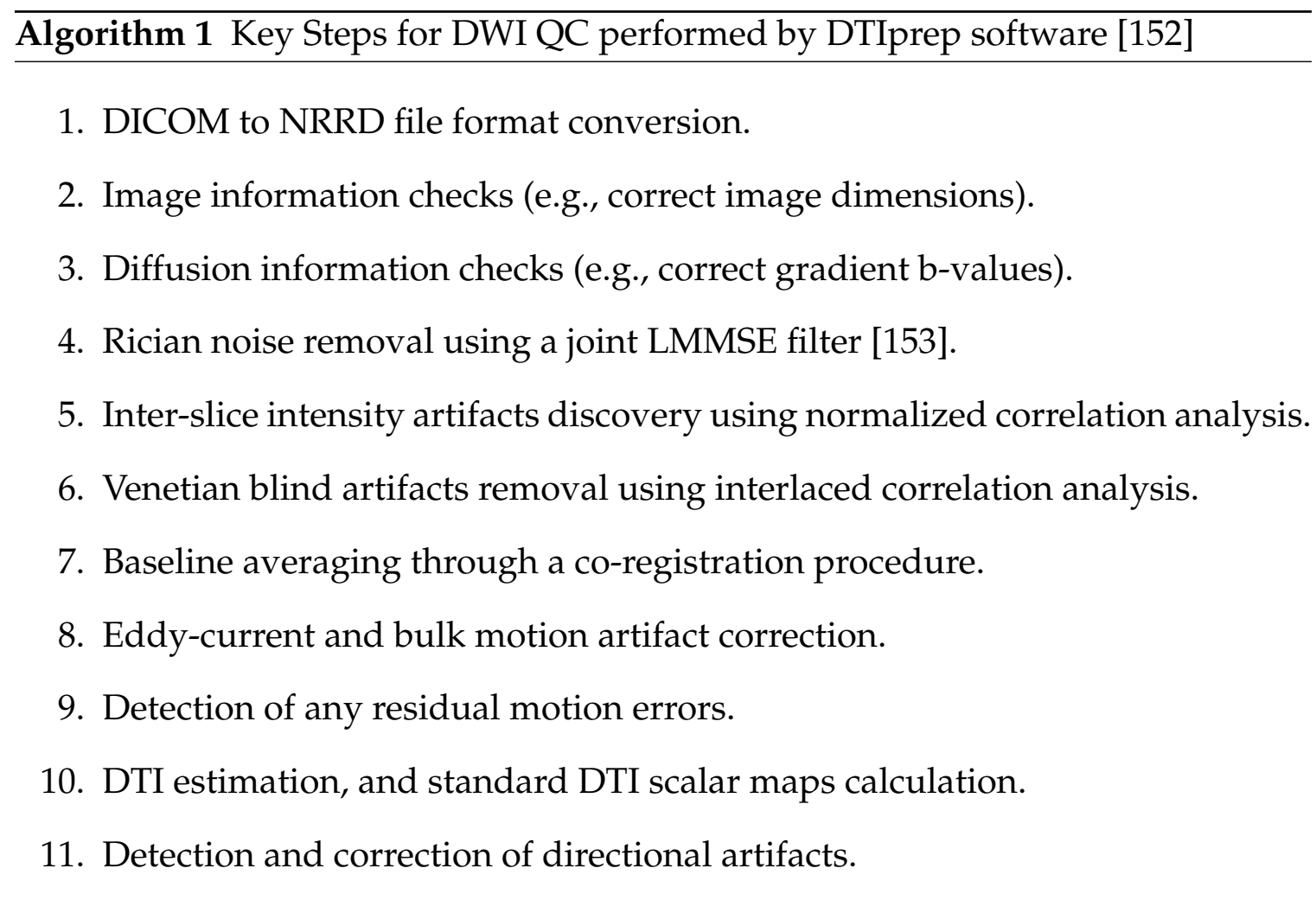

\section{DTI Estimation}

Before any DTI features can be extracted, tensor model estimation from the preprocessed DWI data sets, is performed. As seen in Chapter I, there are many DTI estimation methods, namely, linear least square (LLS), weighted linear least square (WLLS), and nonlinear least square (NLLS). In this thesis, WLLS method was chosen because it takes into consideration the noise profile of the scanned MR images to weight the DWI samples in an intensity based estimation process; thus making WLLS method able to provide more accurate estimates compared with the traditional LLS method. Although NLLS could provide even more accurate estimates, WLLS method was preferred because it is much faster than NLLS 
method. Moreover, the optimization process that exists in NLLS method imposes the risk of falling in a local minima, and such risk does not exist in WLLS estimation method. DTI to DWI estimation using WLLS method was carried out using 3D Slicer software [102]. To account for bad tensors, related to any remaining noise or acquisition artifacts, negative eigenvalues (which are physically meaningless) were shifted.

\section{Brain Extraction}

Brain extraction, or skull stripping process involves the removal of any nonbrain tissues (e.g., eyes, dura, and skull) on brain MR images, which is not an easy task, as the borders between brain and non-brain tissues might be indistinct on MR images (e.g., GM and dura). Brain extraction is an essential preprocessing step in any CAD system that deals with the brain, especially if any form of shape-based analysis is performed (e.g., failing to remove the dura or missing brain parts can result in an overestimation or underestimation of the cortical thickness [154]). In the literature, many brain extraction methods have been proposed. These methods use different techniques, namely, deformable models [155-157], atlas-based [158,159], and label fusion and hybrid algorithms [160-162]. However, most of the existing techniques were designed to work primarily for T1-weighted MR brain images, thus, they are not well suited to extract the brain from diffusion-weighted images directly. Moreover, the majority of the current techniques weer developed to work for adult MR brain images, so they fail to overcome the additional challenges imposed by MR infant images (e.g., reduced contrast and higher noise).

To overcome the mentioned limitations, this thesis proposes a new stochastic brain extraction method, based on a first-order visual appearance model, which guides an edge-preserving image restoration model, to account for intensity inhomogeneity through an energy minimization optimization procedure. 


\section{LCDG Visual Appearance Model}

To accurately approximate the marginal probability distributions of the brain and non-brain tissue, the empirical gray level distribution of a given $\mathrm{g}$ is precisely approximated with a linear combination of discrete Gaussians (LCDG) with positive and negative components [163]. The LCDG restores brain and non-brain transitions more accurately than a conventional mixture of only positive Gaussians. Below, LCDG will be explained in more detail.

Let $\boldsymbol{\Psi}_{\theta}=(\psi(q \mid \theta): q \in \mathbf{Q})$ denote a discrete Gaussian (DG) with parameters $\theta=(\mu, \sigma)$, integrating a continuous 1D Gaussian density with mean $\mu$ and variance $\sigma^{2}$ over successive gray level intervals. The LCDG with two dominant positive DGs and $K_{\mathrm{p}} \geq 4$ positive and $K_{\mathrm{n}} \geq 0$ negative subordinate DGs is [163,164]:

$$
P_{\mathbf{c}, \boldsymbol{\Theta}}(q)=\sum_{k=1}^{K_{\mathrm{p}}} c_{\mathrm{p}: k} \psi\left(q \mid \theta_{\mathrm{p}: k}\right)-\sum_{\kappa=1}^{K_{\mathrm{n}}} c_{\mathrm{n}: \kappa} \psi\left(q \mid \theta_{\mathrm{n}: \kappa}\right),
$$

where all the weights $\mathbf{c}=\left[c_{\mathrm{p}: k}, c_{\mathrm{n}: \kappa}\right]$ are non-negative and meet an obvious con-

straint $\sum_{k=1}^{K_{\mathrm{p}}} c_{\mathrm{p}: k}-\sum_{\kappa=1}^{K_{\mathrm{n}}} c_{\mathrm{n}: \kappa}=1$. All LCDG parameters, including the DGs numbers, are estimated from the mixed empirical distribution to be modeled using the modified EM algorithm [163]. Figure 23 illustrates the final estimated LCDG density for each class.

At the end of this step, a discriminant threshold $\tau$ is calculated in a way that ensures the best separation between the brain and the non-brain voxel signals, as illustrated in Figure 24. This threshold will be used in the next steps to enhance the process of classifying image voxels into either brain or non-brain.

\section{GGMRF Inhomogeneity Reduction Model}

Intensity inhomogeneity of infant brain DWI images limits the accuracy of the existing brain extraction approaches. Therefore, to accurately extract the brain it is important to account for the low frequency intensity inhomogeneity. In the 


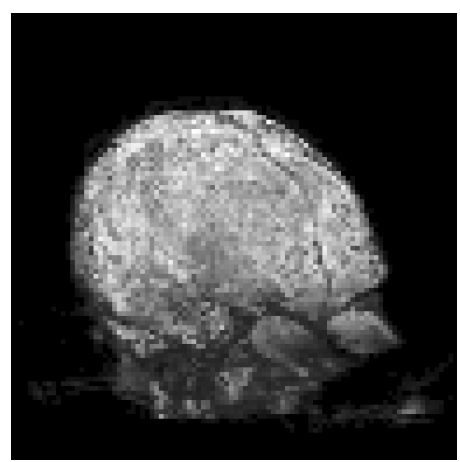

(a) 3D infant brain

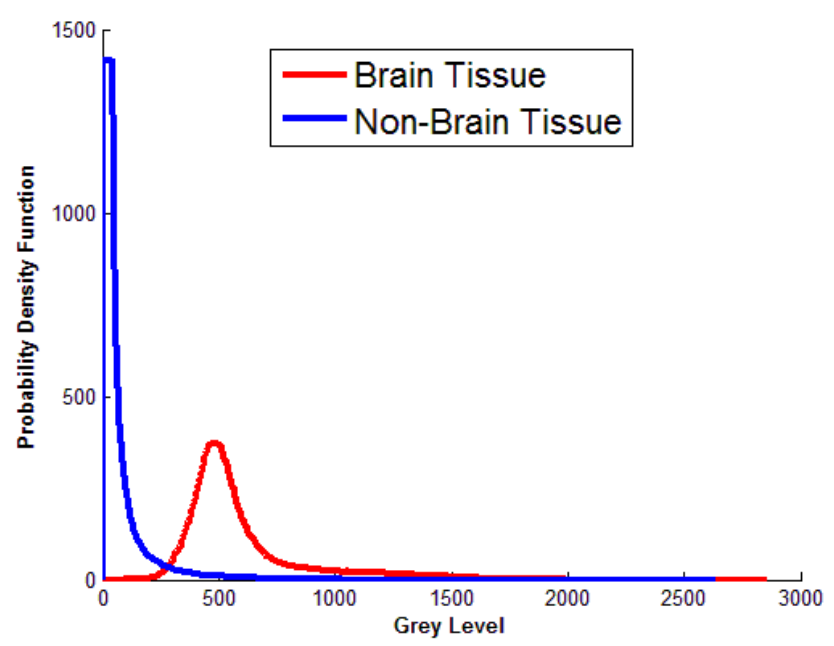

(b) Estimated LCDG model

Figure 23: The final estimated LCDG density for each of the two classes (i.e., brain and non-brain).

proposed brain extraction method, the generalized Gauss-Markov random field (GGMRF) model is used to reduce this inhomogeneity [165], which is applied after DWI data is preprocessed using DTIprep software. In this step, the residual image inconsistencies are removed (smoothed) by accounting for the spatially homogeneous 3D pair-wise interactions between the gray levels of the DWI data. Namely, the gray level values $q \in \mathbf{Q}$ are considered as samples from a 3D GGMRF model [165] of measurements with the voxel 26-neighborhood demonstrated in Figure 25. The continuity of $q$ values of each brain DWI scan is modified by applying the gradient descent algorithm [166] to search for the closest minimum of the 


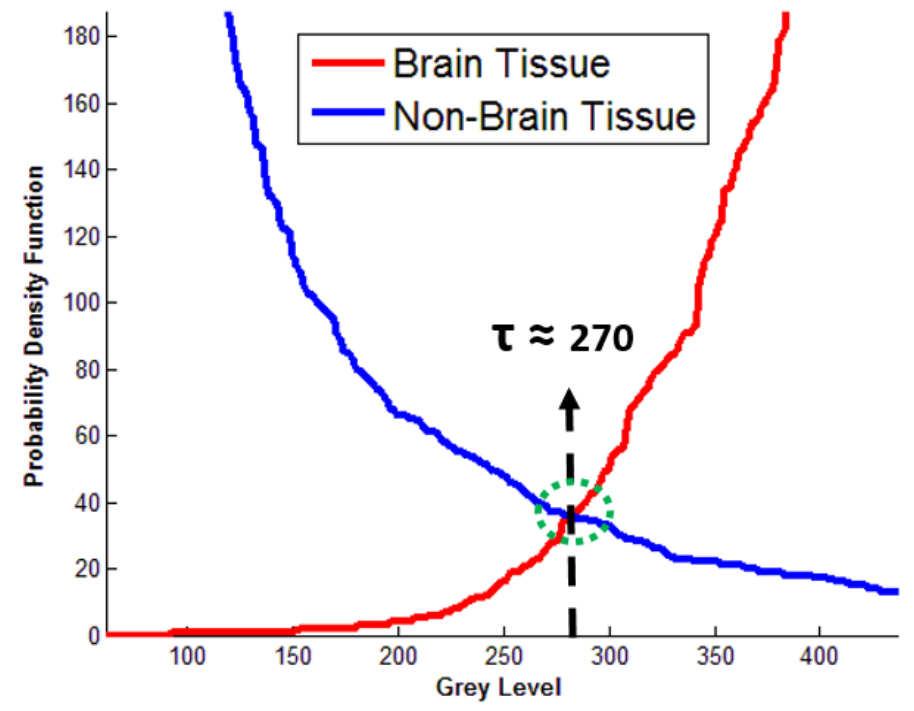

Figure 24: The discriminant threshold, $\tau$, calculation method from the estimated visual appearance model.

following equation:

$$
\widehat{q}_{s}=\arg \min _{\widetilde{q}_{s}}\left[\left|q_{s}-\widetilde{q}_{s}\right|^{\alpha}+\rho^{\alpha} \lambda^{\beta} \sum_{r \in \nu_{s}} \eta_{s, r}\left|\widetilde{q}_{s}-q_{r}\right|^{\beta}\right],
$$

where $q_{s}$ and $\widetilde{q}_{s}$ denote the original gray level values and their expected estimates, respectively, at the observed 3D location, $s=(x, y, z) ; \nu_{s}$ is the 26neighborhood voxel set (Figure 25); $\eta_{s, r}$ is the GGMRF potential, and $\rho$ and $\lambda$ are scaling factors. The parameter $\beta \in[1.01,2.0]$ controls the level of smoothing (e.g., $\beta=2$ for smooth vs. $\beta=1.01$ for relatively abrupt edges). The parameter $\alpha \in\{1,2\}$ determines the Gaussian, $\alpha=2$, or Laplace, $\alpha=1$, prior distribution of the estimator.

To better classify each modified signal as either the brain or non-brain, the voxel signals are nudged additionally towards their most appropriate grouping through incrementing or decrementing them by a bias value of $\epsilon$. The latter was chosen experimentally as $0.5 \%$ of the maximum gray value, in accord with the dis- 


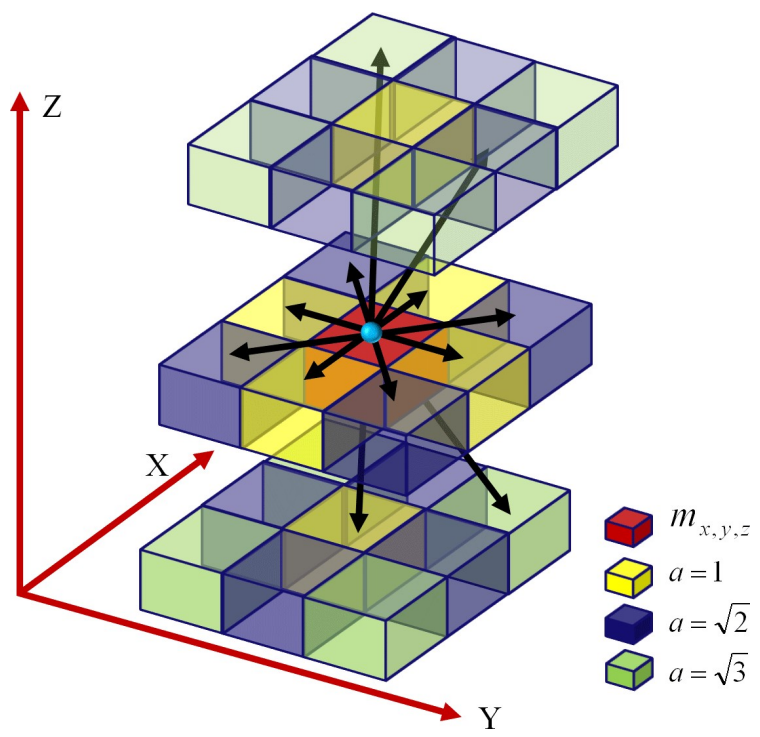

Figure 25: Illustration of the 3D neighborhood system

criminant threshold $(\tau)$ determined from the LCDG model. The larger the bias, the wider the grayscale gap between brain and non-brain in the modified image. While smaller biases may be useful to gradually optimize the separation between the two classes, larger ones sharpen signal gradients and increase the overall spectral noise. After the final modified image is obtained, a 3D region growing is applied, starting from a seed point at the center of the image volume, followed by connected component analysis to calculate the final brain mask, which is used to find the final extracted brain (see Figure 26). The overall data processing procedure of the proposed brain extraction method is summarized in Algorithm 2.

\section{The Joint MGRF Segmentation Model}

Following brain extraction, the next step is to segment different brain structures from the extracted infant brains. To achieve this goal, a novel 3D joint MGRF model is presented in this chapter. An input brain image, $g$, co-aligned to the 


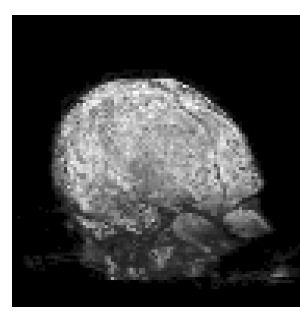

(a) Original brain

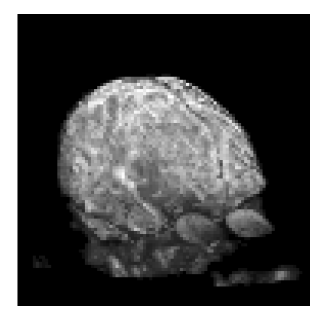

(b) Smoothed brain

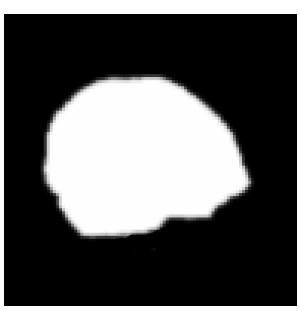

(c) Brain mask

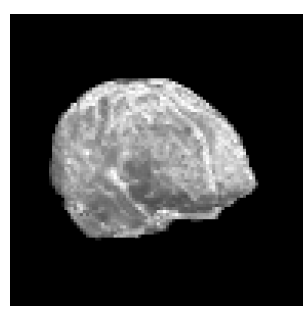

(d) Extracted brain

Figure 26: Illustration of the brain extraction procedure.

training database, and its map, $\mathbf{m}$, are described with a joint probability model: $P(\mathbf{g}, \mathbf{m})=P(\mathbf{g} \mid \mathbf{m}) P(\mathbf{m})$, which combines a conditional distribution of the images given the map $P(\mathbf{g} \mid \mathbf{m})$, and an unconditional probability distribution of maps $P(\mathbf{m})=P_{\mathrm{sp}}(\mathbf{m}) P_{\mathbf{V}}(\mathbf{m})$. Here, $P(\mathbf{g} \mid \mathbf{m})$ is a NMF-based visual appearance model, $P_{\mathrm{sp}}(\mathbf{m})$ is an adaptive shape prior, and $P_{\mathbf{V}}(\mathbf{m})$ is a Gibbs probability distribution with potentials V, which specifies a Markov Gibbs random field (MGRF) model of spatially homogeneous maps $\mathbf{m}$. Details of the model's components are outlined below.

\section{NMF-Based Visual Appearance Model}

Following DTI estimation, a set of different scalar measurements (features) from the estimated diffusion tensor is computed. These DTI-based features provide rich information about the brain anatomy, and would help to make different brain tissues more distinguishable. In this thesis, five different anisotropy features were calculated using 3D Slicer software [102], namely, mean diffusivity (MD), fractional anisotropy (FA), relative anisotropy (RA), axial diffusivity $\left(\lambda_{\|}\right)$, and radial diffusivity $\left(\lambda_{\perp}\right)$. The first two features (FA and RA) will help to separate WM from other brain tissues, while the other three features (MD, $\lambda_{\|}$, and $\lambda_{\perp}$ ) can differentiate between GM and CSF tissues (see Chapter I). However, there is a need to extract the most meaningful information from that large DTI dimensional space.

NMF has been shown to have powerful capabilities in clustering complex 
Algorithm 2 Key Steps for the Proposed Brain Extraction Approach

1. Approximate the marginal intensity distribution of an input image $\mathrm{g}$ using an LCDG model with two dominant modes for the brain and the non-brain classes.

2. Determine the disclaimant threshold, $\tau$, which ensures the best separation between the brain and the non-brain voxel signals.

3. Reduce the inhomogeneity of $\mathrm{g}$ by applying the 3D GGMRF model to each voxel signal $\left(q_{s} ; s \in \mathbf{R}, q \in \mathbf{Q}\right)$ to get the modified image $\widehat{\mathbf{g}}$.

4. Nudge pixels that may fall close the boundary between the brain and the non-brain classes by comparing the signal of modified voxels $\widehat{q}_{s}$ to the threshold $\tau$ obtained in step (2), and either add a small fixed bias $\epsilon$ to or subtract it from these modified signals.

5. Improve $\widehat{\mathrm{g}}$ using gradient descent optimization algorithm [166].

6. Apply 3D region growing followed by connected component analysis to obtain the final results.

data based on extracting features that have the ability to learn the characteristics of data classes [167]. As a consequence, the application of NMF was extended to the field of medical image segmentation in the last few years. In NMF-based image segmentation, a weight matrix $\mathbf{W}$ is calculated to transform a vector from the input space into a new feature space (H-space) through factorizing the input matrix $\mathbf{A}$. NMF has been used in a few DTI-based segmentation systems. For instance, Xie et al. [168] used NMF to segment the spinal cord, corpus callosum, and hippocampus from rates' DTI images by applying the k-means clustering approach [169] to the column vectors of the produced $\mathbf{H}$ matrix in order to associate each data sample to a given cluster. NMF has been used in some segmentation problems with a promising success; however, further investigations are still needed to verify NMF segmentation potential.

In this work, in order to more accurately segment different brain structured from DTI data, feature fusion based on NMF is applied to extract new meaningful 
features from the large dimensional DTI feature space (A), which consists of one appearance feature (b0) and five anisotropy features (MD, FA, RA, $\lambda_{\|}, \lambda_{\perp}$ ). In particular, NMF is used in this segmentation framework to find the weights for each input DTI feature (W) in order to create a new feature space $(\mathbf{H})$, in which dimensionality is reduced, information from the training data set is encoded, and different classes is better separated. Using NMF, the input data matrix $\mathbf{A} \in \mathbb{R}^{+I \times X Y Z}$ can be factorized into two matrices:

$$
\mathrm{A} \approx \mathrm{WH}
$$

where $\mathbf{W} \in \mathbb{R}^{+I \times J}$ contains the basis voxels as its columns, and $\mathbf{H} \in \mathbb{R}^{+J \times X Y Z}$ is new feature encoding of voxels [167]. To process the 3D DTI features using NMF, each DTI feature for each voxel $(x, y, z)$ in the 3D brain is converted to vectors in the input data matrix $\mathbf{A}$. $\mathbf{W}$ and $\mathbf{H}$ were calculated by minimizing the Euclidean distance between $\mathbf{A}$ and $\mathbf{W H}$ with the constraint that $\mathbf{W}$ and $\mathbf{H}$ contained only non-negative values. This results in this constrained optimization problem:

$$
\begin{array}{ll}
\underset{\mathbf{W}, \mathbf{H}}{\operatorname{minimize}} & \frac{1}{2}\|\mathbf{A}-\mathbf{W H}\|^{2} \\
\text { subject to } & \mathbf{W}, \mathbf{H} \geq 0,
\end{array}
$$

and since the advent of NMF, several methods have been used to optimize Equation (22). The most prominent algorithms have been multiplicative update, projected gradient descent (PGD), and alternating least square (ALS) [170]. In this chapter, the ALS algorithm was used because of its high speed and flexibility compared with other competing algorithms. The ALS algorithm takes advantage from the fact that, although Equation (22) is not convex in both $\mathbf{W}$ and $\mathbf{H}$, it is convex in either $\mathbf{W}$ or $\mathbf{H}$ when the other is considered as constant. Therefore, a first least square step is required to find one matrix, followed by another least square step in an altering pattern. The basic ALS algorithm for NMF is presented in Algorithm 3 [170]. 
Algorithm 3 Key Steps of the ALS Algorithm for NMF [170]

1. Initialize $\mathbf{W}$ as a random dense matrix.

2. Solve for $\mathbf{H}$ in the matrix equation $\mathbf{W}^{T} \mathbf{W H}=\mathbf{W}^{T} \mathbf{A}$.

3. Set the negative values in $\mathbf{H}$ to zero.

4. Solve for $\mathbf{W}$ in the matrix equation $\mathbf{H H}^{T} \mathbf{W}^{T}=\mathbf{H A}^{T}$.

5. Set the negative values in $\mathbf{W}$ to zero.

6. Repeat steps (2-5) till the predefined maximum number of iterations is reached.

In the proposed framework, NMF was performed on a input data matrix that had a $I^{\text {th }}$ dimensional, one dimension for each calculated feature, and a column vector for each voxel in the training volumes. The resulting $\mathbf{W}$ was used as the basis vectors to transform new feature vectors into the new $J^{\text {th }}$-dimensional space. The resulting $\mathbf{H}$ was used to find the $J$-dimensional centroids corresponding to each brain label $\left(C_{l} ; l \in \mathbf{L}\right)$. In the testing phase, a new input data matrix was created (B) with the same $I^{\text {th }}$-dimensional for each feature as in the training phase, and a column vector for each voxel in the test volume. The new $J^{\text {th }}$-dimensional vectors corresponding to these new input vectors were calculated by multiplying them by the psuedo-inverse of $\mathbf{W}$ [171] (see Figure 27). Mathematically, this is given by:

$$
\mathbf{H}_{B}=\mathbf{W}^{\dagger} \mathbf{B}
$$




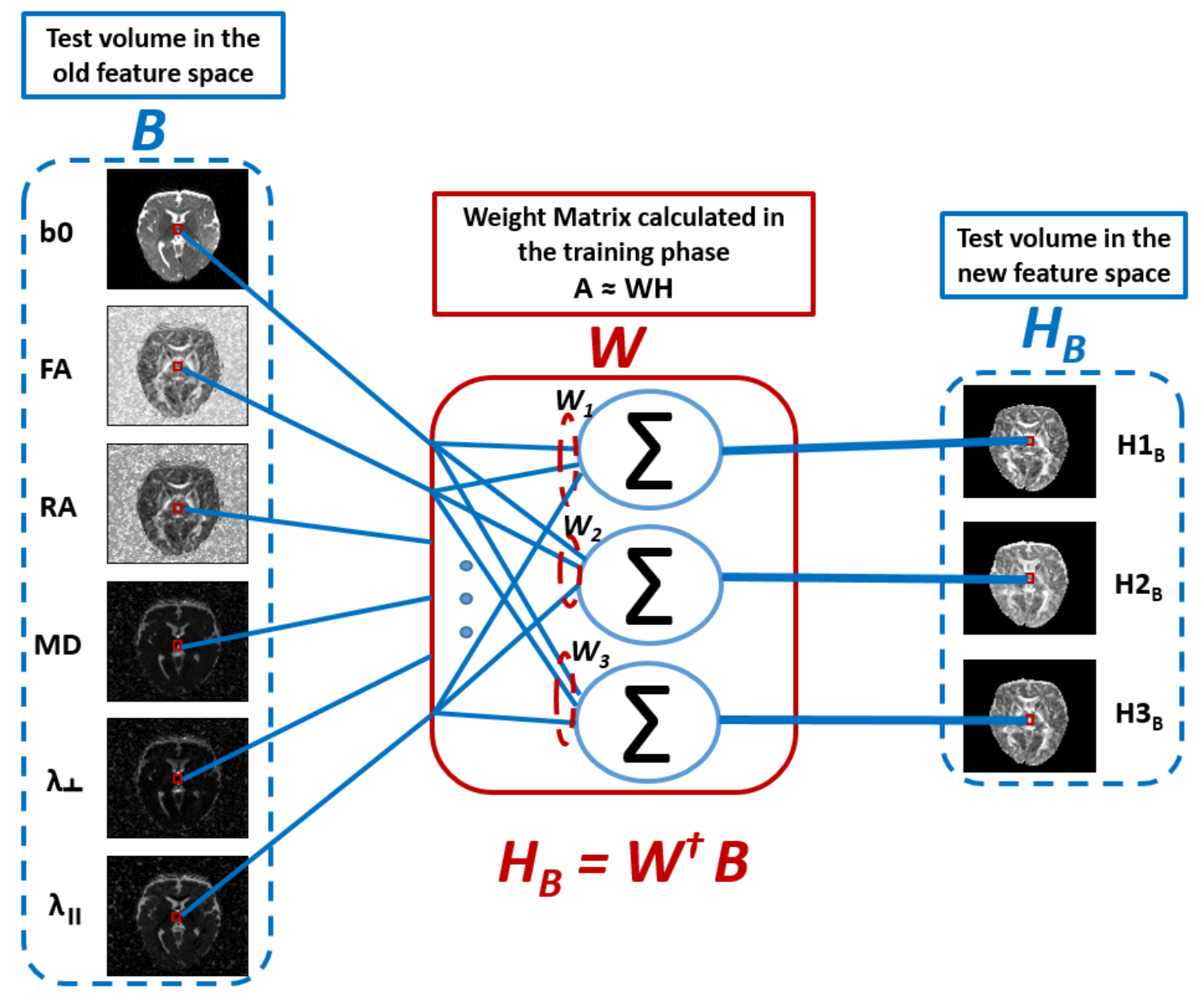

Figure 27: Block diagram for of proposed NMF-based feature fusion.

The proposed segmentation framework accounts for the visual appearance of the new NMF fused features. To model the visual appearence of these features, a $K$-means classifier [172] was used with the classes $J$-dimensional centroids $\left(C_{l}\right.$; $l \in \mathbf{L})$ that were calculated in the $H$-space during the training phase. For each voxel $(x, y, z) \in \mathbf{R}$ in the testing volume $\mathbf{B}$, a new $J$-dimensional test vector $H_{B: x, y, z}$ is formed using Equation (23). The labels probabilities associated with $H_{B: x, y, z}$ were calculated based on the Euclidean distance $d_{l}\left(T_{B: x, y, z}\right)$ from the test vector $H_{B: x, y, z}$ to each of the centroids $C_{l} ; l \in \mathbf{L}$ (see Figure 28). The NMF-based probabilities for 
brain label $l \in \mathbf{L}$, and voxel $(x, y, z) \in \mathbf{R}$ is defined as:

$$
P_{x, y, z}(\mathbf{g} \mid \mathbf{m}=l)=\frac{\frac{1}{d_{l}\left(H_{B: x, y, z}\right)}}{\sum_{l=1}^{L} \frac{1}{d_{l}\left(H_{B: x, y, z}\right)}}
$$

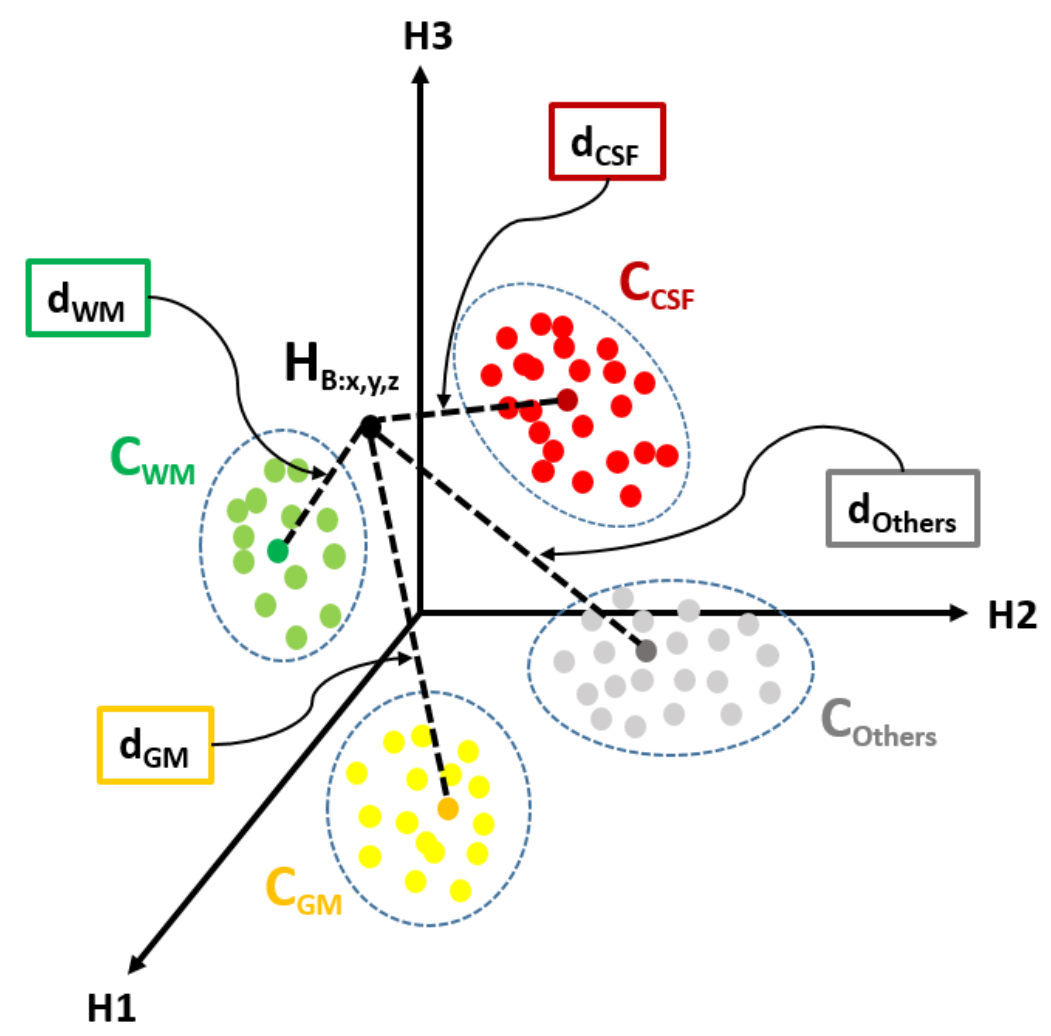

Figure 28: Illustration of the Euclidean distances used in calculating the NMFbased probabilities.

Adaptive Shape Model

To enhance the segmentation accuracy, expected shapes of each brain label are constrained with an adaptive probabilistic shape prior. To create the shape (atlas) database, a training set of images are collected for different subjects (10 data sets) with their new NMF fused features. They are co-aligned by 3D affine transfor- 
mations with 12 degrees of freedom (3 for the 3D translation, 3 for the 3D rotation, 3 for the 3D scaling, and 3 for the 3D shearing) in a way that maximizes their mutual information (MI) [173]. The shape priors are spatially variant independent random fields of region labels for the co-aligned data:

$$
P_{\mathrm{sp}}(\mathbf{m})=\prod_{(x, y, z) \in \mathrm{R}} p_{\mathrm{sp}: x, y, z}\left(m_{x, y, z}\right)
$$

where $p_{\mathrm{sp}: x, y, z}(l)$ refers to the voxel-wise empirical probabilities for each brain label $l \in \mathbf{L}$. To generate the ground truth labels for the atlas, the infant tissue probability maps provided by IDEA lab [174] was used with the unified segmentation algorithm [175], implemented in statistical parametric mapping (SPM) software [176], to segment the non-diffusion (b0) scans of the training data sets. Then, an MR expert refined the generated initial segmentation to produce the final brain labels.

For each input subject data to be segmented, the shape prior is constructed by an adaptive process guided by the visual appearance of its NMF fused features. First, the normalized cross correlation (NCC) similarity coefficient was used to select the subject from the atlas database that has the best match with the input subject (i.e., highest similarity). The selected subject is then used as a reference prototype to co-align the input subject using the 3D affine transformation described above. In order to estimate the shape prior probabilities for each voxel in the test subject (see Figure 29), the steps described in Algorithm 4 were followed.

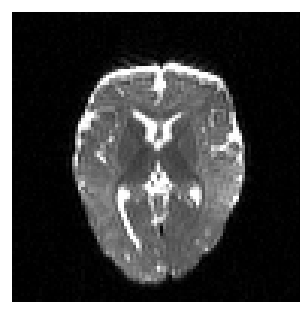

(a) b0 refrence

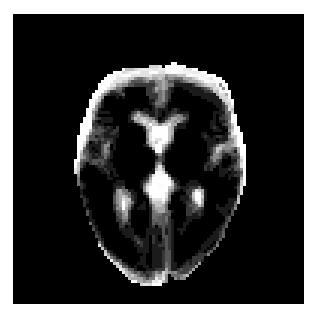

(b) $P_{c s f}$

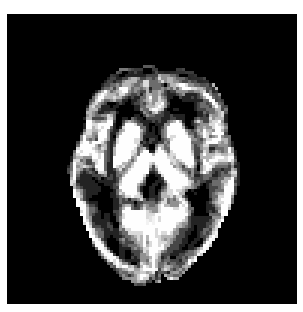

(c) $P_{g m}$

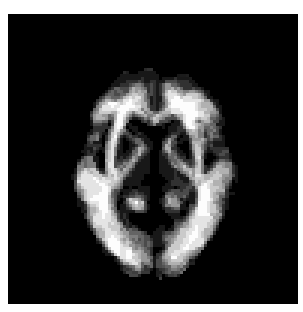

(d) $P_{w m}$

Figure 29: The shape prior probabilities estimated by the proposed adaptive shape model. (a) b0 reference image, (b) CSF prior probability, (c) GM prior probability, and (d) WM prior probability. 
Algorithm 4 Key Steps for Creating the Adaptive Shape Model

1. Construct the atlas database through The co-alignment of the training brains (NMF-fused features and manual segmentation of each of the preprocessed brains).

2. Use the NCC to measure the similarity between the test subject and each subject in the atlas database, and choose the database subject that has the highest similarity to act as the reference in the registration process.

3. Register the test subject to the selected reference subject using 3D affine transformations with 12 degrees of freedom that maximizes their MI [173].

4. For each voxel in the test subject, calculate its shape prior probability according to the following steps:

(a) Use the obtained transformation matrix (T) to transform each voxel to the atlas database domain.

(b) Construct a 3D window with initial size of $N_{1 i} \times N_{2 i} \times N_{3 i}$.

(c) Search inside the window for voxels with corresponding grey values (NMF-fused features) in all training data sets.

(d) If needed, increase the window size and redo the search until a nonempty result is found.

(e) Create the labels probabilities based on the relative occurrence of each label from the search results.

3D Spatial Interaction MGRF Model

In order to overcome noise effects and to ensure segmentation homogeneity; spatially homogeneous 3D pair-wise interactions between the region labels are additionally incorporated in the proposed segmentation model. These interactions are calculated using the popular Potts model (i.e., an MGRF with the nearest 26neighbors of the voxels and analytic bi-valued Gibbs potentials, as illustrated in Figure 30) because only the coincidence of the labels is taken into account. The utilized second-order 3D MGRF model of the region map $\mathbf{m}$ is defined as [177]: 


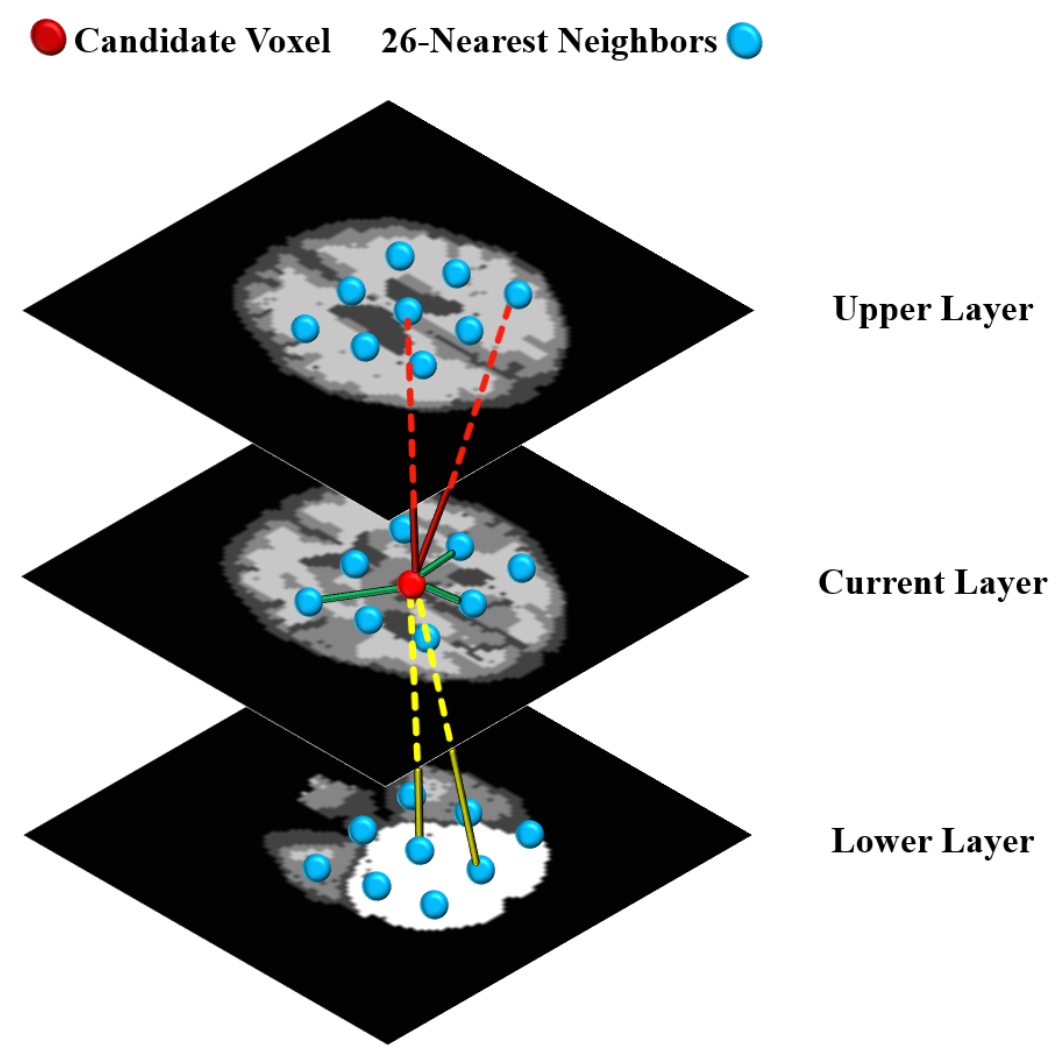

Figure 30: Samples of different pair-wise cliques for the 26-neighborhood system. Graph cliques are shown in different colors for visualization purpose.

$$
P_{\mathbf{V}}(\mathbf{m})=\frac{1}{Z_{\nu_{s}}} \exp \sum_{(x, y, z) \in \mathbf{R}} \sum_{(\xi, \eta, \zeta) \in \nu_{s}} \mathbf{V}\left(m_{x, y, z}, m_{x+\xi, y+\eta, z+\zeta}\right),
$$

where $Z_{\nu_{s}}$ is the normalization factor which can to be approximated by the Equation [178]:

$$
Z_{\nu_{s}} \approx \exp \sum_{(x, y, z) \in \mathbf{R}} \sum_{(\xi, \eta, \zeta) \in \nu_{s}} \sum_{l \in \mathbf{L}} \mathbf{V}\left(l, m_{x+\xi, y+\eta, z+\zeta}\right),
$$

and $V$ is the bi-valued Gibbs potential, which depends on whether the nearest pair of labels are equal or not: 


$$
\mathbf{V}\left(m_{s_{1}}, m_{s_{2}}\right)= \begin{cases}v_{\mathrm{eq}} & \text { if } m_{s_{1}}=m_{s_{2}} \\ v_{\mathrm{ne}} & \text { otherwise }\end{cases}
$$

where $m_{s_{i}}$ is the region map label at the voxel $s_{i}=\left(x_{i}, y_{i}, z_{i}\right)$, and $v_{\text {eq }}$ and $v_{\text {ne }}$ are the estimated potentials in the case of equal and non-equal labels, respectively. The initial region map results in an approximation with the following analytical maximum likelihood estimates of the potentials [177]:

$$
v_{\mathrm{eq}}=-v_{\mathrm{ne}} \approx 2 f_{\mathrm{eq}}(\mathbf{m})-1
$$

where $f_{a, e q}(\mathbf{m})$ is the relative frequency of equal labels in the voxel pairs $\{((x, y, z),(x+$ $\left.\xi, y+\eta, z+\zeta)):(x, y, z) \in \mathbf{R} ;(x+\xi, y+\eta, z+\zeta) \in \mathbf{R} ;(\xi, \eta, \zeta) \in \nu_{s}\right\}$. These estimates allow for computing the voxel-wise probabilities $p_{x, y, z}\left(m_{x, y, z}=l\right)$ of each brain label; $l \in \mathbf{L}$. In total, the complete segmentation steps of the proposed framework are summarized in Algorithm 5. For completeness, the analytical estimation of the bi-valued Gibbs potentials is driven in the Appendix.

\section{Performance Evaluation Metrics}

To evaluate the segmentation accuracy, we used three metrics, namely, (i) the Dice Similarity Coefficient (DSC), (ii) the 95-percentile modified Hausdorff distance $\left(H_{95}\right)$, and (iii) the absolute volume difference (AVD) [179]. The following subsections will describe each used metric in more detail.

\section{Dice Similarity Coefficient}

The Dice similarity coefficient (DSC) measures set agreement between two sets $(\mathrm{S}, \mathrm{G})$, and is defined as the union size of the two sets divided by the average size of the two sets: 
Algorithm 5 Key Steps for the Proposed Segmentation Approach

1. Detect artifacts, correct motion and eddy current distortions and remove images with large artifacts using DTIprep software [152] (see Algorithm 1).

2. Derive DTI from DWI, and extract five DTI anisotropy features (MD, FA, RA, $\left.\lambda_{\|}, \lambda_{\perp}\right)$ using 3D Slicer [102].

3. Use the proposed automated brain extraction approach (Algorithm 2) to remove any non-brain tissues from DWI brain images.

4. Create a new NMF input data matrix B from the obtained DTI features in addition to the b0 volume.

5. Use the weight matrix $\mathbf{W}$ (calculated in the training phase according to Algorithm 3) to transform B from old $I^{\text {th }}$-dimensional space to the new $J^{\text {th }}$ dimensional space, according to Equation (23).

6. Create the subject-specific shape prior model according to the steps in Algorithm 4 .

7. Form an initial region map $\mathbf{m}$ using the NMF-based probabilities and prior adaptive shape of each label.

8. Find the Gibbs potentials for the MGRF model from the initial map $\mathbf{m}$.

9. Improve the region map $m$ using voxel-wise Bayes classifier after integrating the three descriptors in the proposed joint MGRF model..

$$
\operatorname{DSC}(\mathbf{S}, \mathbf{G})=\frac{2|\mathbf{S} \cap \mathbf{G}|}{\mathbf{S} \cap \mathbf{G}+\mathbf{S} \cup \mathbf{G}} \times 100
$$

In segmentation validation, the DSC is usually expressed in terms of false positive (FP), false negative (FN), true negative (TN), and true positive (TP) counts, which were obtained by comparing the segmentation results to the ground truth (gold standard) (see Figure 31). These values can be used to calculate the DSC as shown by [179]:

$$
\mathrm{DSC}=\frac{2 T P}{2 T P+F P+F N} \times 100 .
$$


A DSC value of zero indicates no overlap; a value of one indicates ideal segmentation. Higher numbers indicate better segmentation, which means that the segmentation results match the ground truth better than results with lower DSC values.

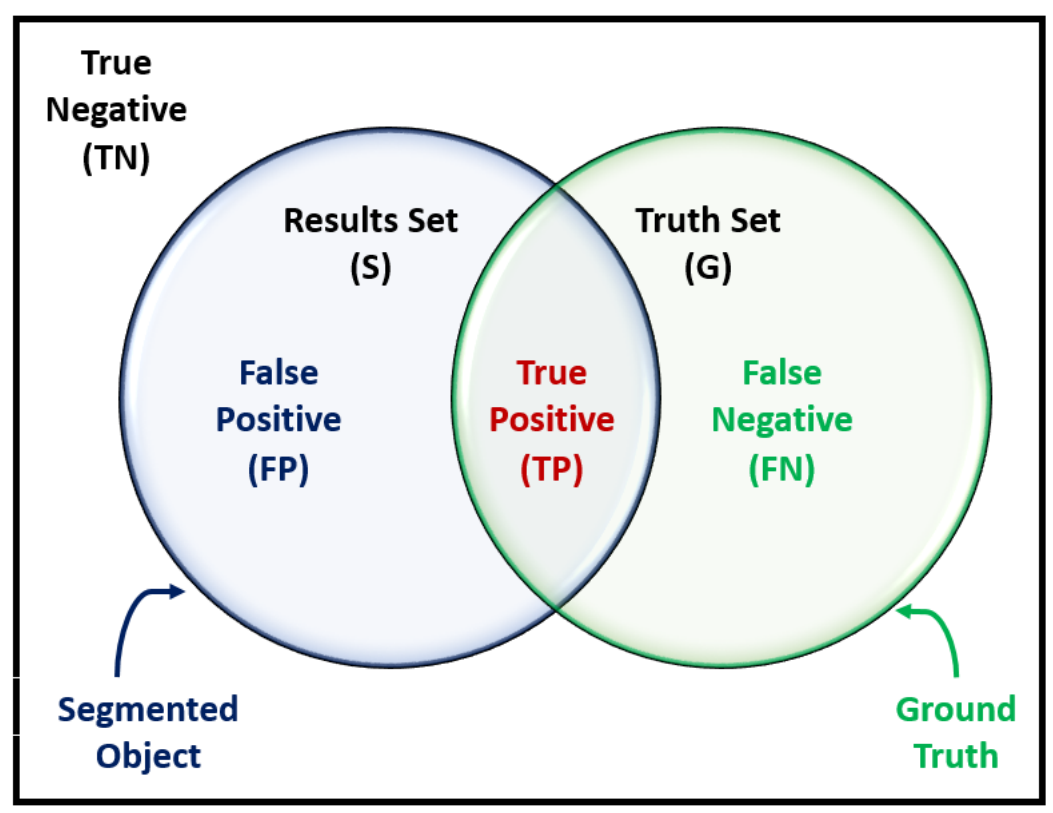

Figure 31: Diagram illustrating the meaning of segmentation errors, namely, true positive (TP), false positive (FP), true negative (TN), and false positive (FP). These segmentation errors, obtained by comparing the segmented and the ground truth objects, are used to calculate the dice similarity coefficient (DSC).

\section{Modified Hausdorff Distance}

Distance measures are another type of performance metric used for evaluating segmentation methods. The Euclidean distance is often utilized, but another common measure is the Hausdorff distance $(\mathrm{H})$. The $\mathrm{H}$ value from a set $\mathbf{S}$ to a set $\mathrm{G}$ is defined as the maximum distance of the set $\mathrm{S}$ to the nearest point in the set $\mathrm{G}$ (see Figure 32) [179]: 


$$
H(\mathbf{S}, \mathbf{G})=\max _{s \in \mathbf{S}}\left\{\min _{g \in \mathbf{G}}\{d(s, g)\}\right\},
$$

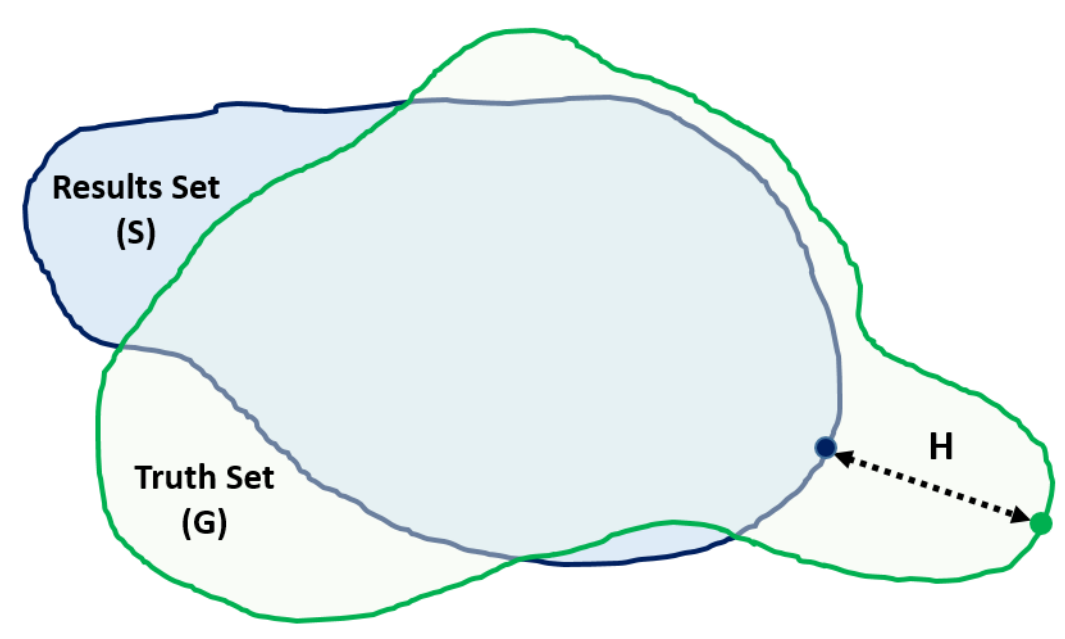

Figure 32: A schematic illustration for the Hausdorff distance $(\mathrm{H})$ calculation.

where $s$ and $g$ are points of sets $\mathbf{S}$ and $\mathbf{G}$, respectively, and $d(s, g)$ is Euclidean distance between these points. The bidirectional Hausdorff distance, denoted by $H_{B i}(\mathbf{S}, \mathbf{G})$, between the segmented region (S) and its ground truth $(\mathbf{G})$ is defined as:

$$
H_{B i}(\mathbf{S}, \mathbf{G})=\max \{H(\mathbf{S}, \mathbf{G}), H(\mathbf{G}, \mathbf{S})\} .
$$

The smaller the distance, the better the segmentation. The ideal case with perfect segmentation is when the bidirectional Hausdorff distance is equal to 0 . In this thesis, to eliminate the effect of segmentation outliers, the 95-percentile modified Hausdorff distance $\left(H_{95}\right)$ was used to assess the proposed segmentation framework accuracy. 


\section{Absolute Volume Difference}

In addition to the DSC and $H_{95}$ metrics, a volumetric metric is also used to assess the accuracy of the segmentation. The percentage absolute volumetric difference (AVD) is defined as the ratio of the absolute difference between the original volume and the segmented volume, to the original volume (see Figure 33) [179]:

$$
\mathrm{AVD}=\frac{\left|V_{s}-V_{g}\right|}{V_{g}} \times 100
$$

where $V_{s}$ and $V_{g}$ are the segmented volume and the ground truth volume, respectively, and each were calculated by multiplying the number of labelled voxels by the voxel dimensions [179]. The smaller the AVD, the better the segmentation.

Results Set

(S)

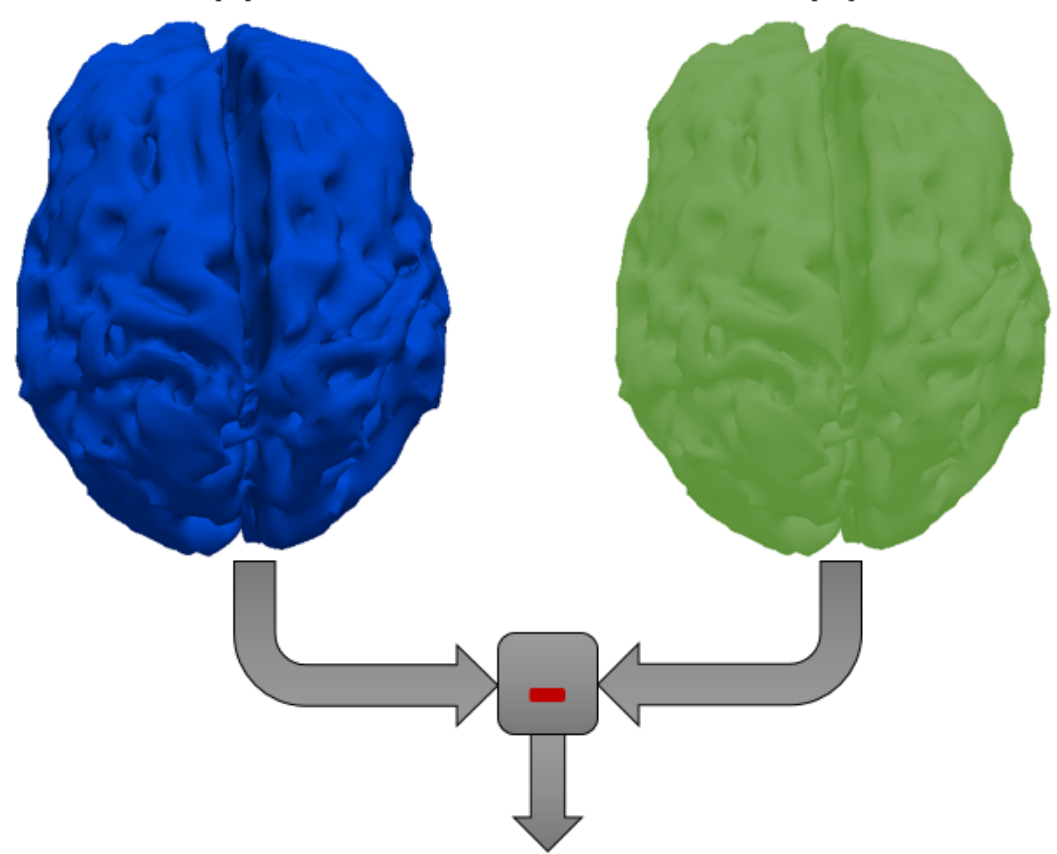

AVD

Figure 33: A schematic illustration for the absolute volume difference (AVD) estimation. 
E Experimental Results and Evaluation

1 Participants and Image Acquisition

This study included data from the Infant Brain Imaging Study (IBIS) [180], which is the parent network of an ongoing study of infants at risk for autism. The study participants are six-month-old infants with high risk of developing autism spectrum disorders (ASDs). These infants were seen for follow-up assessments at 12 and 24 months of age, and the final assessment was made at age 24 months. The high-risk infants were divided into two groups: ASD negative (below the ASD cutoff) and ASD-positive (above the cutoff). Diffusion weighted MRI brain scans were obtained from a 3-T Siemens TIM Trio scanners (Siemens Medical Solutions, Malvern, PA.) using the following parameters: field of view of: $190 \mathrm{~mm}$, number of slices: 75-81, a slice thickness: $2 \mathrm{~mm}$, voxel resolution: $2 \times 2 \times 2 \mathrm{~mm}^{3}$, TR: 12,800-13,300 ms, TE: $102 \mathrm{~ms}$, variable $\mathrm{b}$ values between 0 and 1,000 s/mm $\mathrm{mm}^{2}, 25$ gradient directions, and a scan time of 5-6 minutes.

\section{Segmentation Results}

Performance assessment of the segmentation results were carried out through applying the proposed techniques on 54 diffusion weighted infant MR brain data sets, and evaluated using 9 data sets with a manually segmented ground truth, obtained by an MR expert. The final goal of the proposed segmentation technique is to separate infant DTI brain images into four classes: WM, GM, CSF, and other brain tissues. A step-by-step of the proposed segmentation framework is demonstrated in Figure 35. The input DWI data (Figure 35 (a)) is initially preprocessed using DTIprep software [152] to remove scan artifacts and correct motion and eddy current distortions. Then, the proposed brain extraction method was used to remove any non-brain tissues (e.g., skull and eyes) (Figure 35 (b)). An initial segmentation is achieved using the constructed adaptive shape model and the NMF-based 
visual appearance model (Figure 35 (c)). To obtain the final segmentation, the initial results were refined using the proposed three descriptors (NMF-based visual appearance model, 3D spatial model, and adaptive shape model) as shown in Figure 35 (d). Figure 34 shows the 3D results of the proposed segmentation approach.

The performance of the proposed segmentation framework was evaluated using three performance metrics: (i) the Dice similarity coefficient (DSC), (ii) the 95-percentile modified Hausdorff distance $\left(H_{95}\right)$, and (iii) the percentage absolute volume difference (AVD) [179]. Metrics were computed by comparing a ground truth segmentation to results from the proposed segmentation technique. The detailed segmentation results for each subject are given in Tables 1, 2, and 3. In addition, a summary of the segmentation results, represented by the mean \pm standard deviation values, is provided in Table 4. As demonstrated in Table 4, the DSC for segmentation of the Brain, WM, GM, and the CSF are $96.64 \pm 1.15 \%, 95.23 \pm 1.18 \%$, $89.92 \pm 2.86 \%$, and $87.96 \pm 3.31 \%$, respectively, which confirms the high accuracy of the proposed segmentation techniques. Experiments show that the proposed accurate identification of the joint MGRF model demonstrates promising results in segmenting GM and WM brain tissues from infant DTI images. The present implementation in the $\mathrm{C}++$ programming language on a Dell precession T7500 workstation (3.33Ghz Intel quad-core with 48GB RAM) takes about $8.1 \pm 2.53 \mathrm{sec}$ to process each test subject.

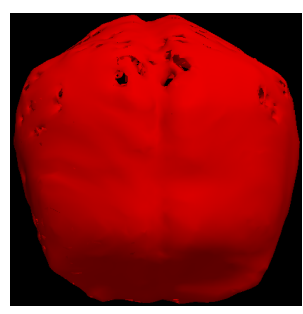

(a) CSF

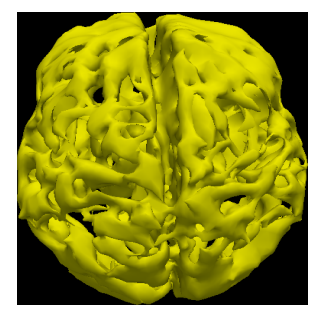

(b) GM

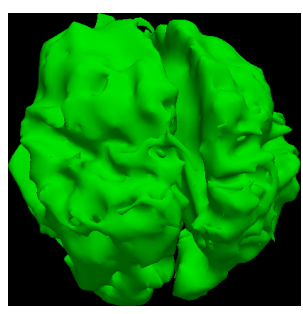

(c) WM

Figure 34: 3D visualization for the segmentation results. 
Table 1

Accuracy of the proposed segmentation approach for each subject using dice similarity coefficient (DSC $(\%))$.

\begin{tabular}{|c|c|c|c|c|c|c|c|c|c|}
\hline \hline Subject No. & $S_{1}$ & $S_{2}$ & $S_{3}$ & $S_{4}$ & $S_{5}$ & $S_{6}$ & $S_{7}$ & $S_{8}$ & $S_{9}$ \\
\hline CSF & 95.40 & 89.07 & 83.66 & 87.63 & 86.19 & 88.49 & 87.63 & 88.57 & 85.02 \\
\hline GM & 92.60 & 91.64 & 88.70 & 92.74 & 88.81 & 90.77 & 84.26 & 91.94 & 87.75 \\
\hline WM & 95.14 & 95.96 & 95.51 & 93.14 & 95.37 & 96.31 & 93.59 & 96.25 & 95.89 \\
\hline Others & 96.44 & 92.06 & 85.89 & 93.54 & 91.16 & 93.85 & 93.14 & 80.12 & 92.81 \\
\hline Brain & 98.85 & 97.47 & 94.90 & 97.20 & 96.70 & 97.43 & 96.92 & 96.07 & 96.38 \\
\hline
\end{tabular}

Table 2

Accuracy of the proposed segmentation approach for each subject using the 95percentile modified Hausdorff distance $\left(H_{95}(\mathrm{~mm})\right)$.

\begin{tabular}{|c|c|c|c|c|c|c|c|c|c|}
\hline \hline Subject No. & $S_{1}$ & $S_{2}$ & $S_{3}$ & $S_{4}$ & $S_{5}$ & $S_{6}$ & $S_{7}$ & $S_{8}$ & $S_{9}$ \\
\hline CSF & 1.98 & 2.00 & 3.44 & 2.80 & 2.81 & 1.98 & 2.81 & 1.98 & 2.00 \\
\hline GM & 1.98 & 1.98 & 2.00 & 1.98 & 2.00 & 1.98 & 2.00 & 1.98 & 1.98 \\
\hline WM & 1.98 & 1.98 & 1.98 & 2.00 & 1.98 & 1.98 & 1.98 & 1.98 & 1.98 \\
\hline Others & 4.00 & 4.00 & 5.97 & 9.22 & 10.00 & 3.44 & 5.95 & 9.76 & 4.43 \\
\hline Brain & 2.80 & 4.00 & 5.63 & 12.00 & 7.19 & 2.81 & 13.24 & 6.88 & 5.60 \\
\hline
\end{tabular}

Table 3

Accuracy of the proposed segmentation approach for each subject using the percentage absolute volume difference $(\operatorname{AVD}(\%))$.

\begin{tabular}{|c|c|c|c|c|c|c|c|c|c|}
\hline \hline Subject No. & $S_{1}$ & $S_{2}$ & $S_{3}$ & $S_{4}$ & $S_{5}$ & $S_{6}$ & $S_{7}$ & $S_{8}$ & $S_{9}$ \\
\hline CSF & 2.57 & 8.14 & 16.93 & 7.05 & 5.95 & 2.90 & 4.18 & 3.53 & 3.66 \\
\hline GM & 9.63 & 7.48 & 11.62 & 7.43 & 11.48 & 8.84 & 14.04 & 8.25 & 9.97 \\
\hline WM & 8.37 & 3.59 & 4.74 & 8.60 & 4.43 & 3.86 & 4.84 & 3.83 & 4.19 \\
\hline Others & 3.73 & 0.42 & 21.12 & 5.79 & 3.49 & 8.51 & 3.43 & 27.23 & 7.93 \\
\hline Brain & 1.69 & 0.81 & 8.39 & 1.52 & 0.70 & 1.27 & 0.68 & 5.27 & 2.64 \\
\hline
\end{tabular}




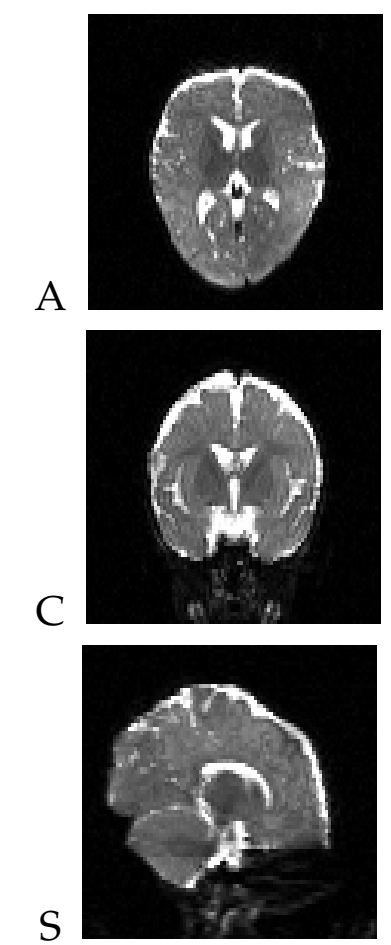

(a)
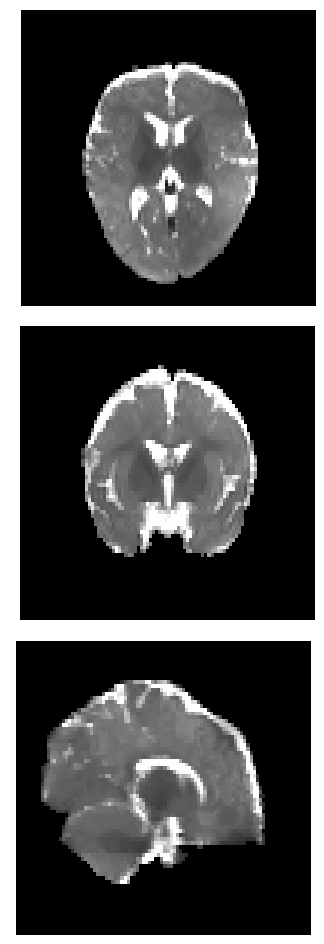

(b)
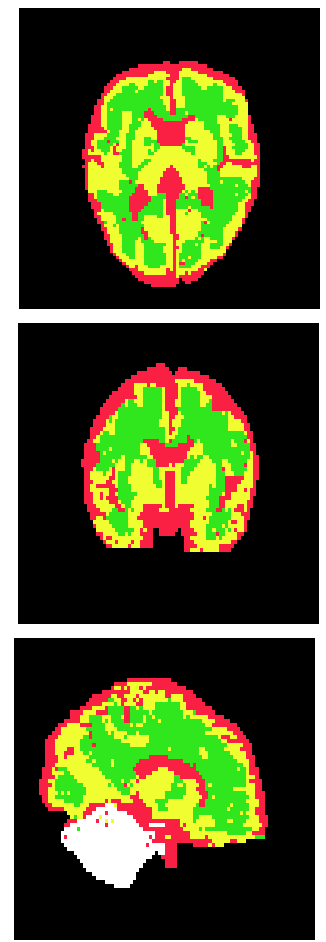

(c)
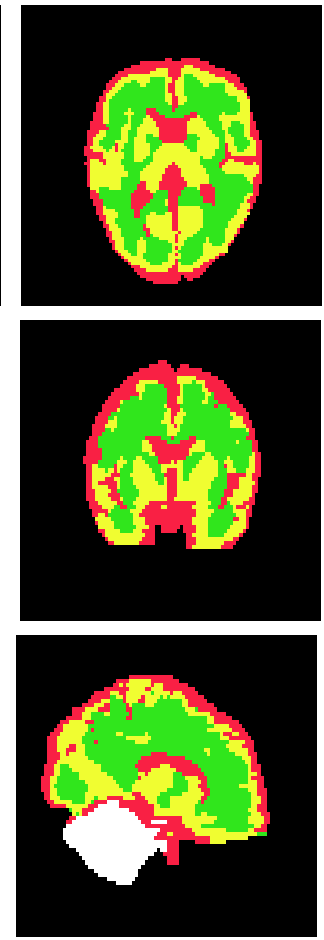

(d)

Figure 35: Segmentation results of the proposed approach. The segmentation is performed in $3 \mathrm{D}$, and the results are projected onto $2 \mathrm{D}$ axial $(\mathrm{A})$, coronal $(\mathrm{C})$, and sagittal (S) planes for visualization: (a) 2D profile of the original b0 scan images, (b) b0 scan images after preprocessed using DTIprep software [152], and brain extraction using the proposed method, (c) initial segmentation using an NMF-based visual appearance model and adaptive shape model, and (d) final segmentation results using the proposed three models. Note that CSF, GM, WM, and other nonbrain tissues are shown in red, yellow, green, and white, respectively.

Table 4

Summary of the segmentation results using DSC $(\%), H_{95}(m m)$, and AVD(\%), represented by the mean \pm standard deviation values.

\begin{tabular}{cccccc}
\hline \hline Metric & CSF & GM & WM & Others & Brain \\
\hline DSC (\%) & $87.96 \pm 3.31$ & $89.92 \pm 2.86$ & $95.23 \pm 1.18$ & $92.81 \pm 5.26$ & $96.64 \pm 1.15$ \\
$H_{95}(m m)$ & $2.42 \pm 0.56$ & $1.98 \pm 1.07$ & $1.98 \pm 0.01$ & $6.60 \pm 2.75$ & $7.17 \pm 3.96$ \\
AVD $(\%)$ & $6.10 \pm 4.70$ & $9.85 \pm 2.34$ & $5.15 \pm 2.03$ & $9.74 \pm 9.65$ & $2.66 \pm 2.80$
\end{tabular}




\section{F Conclusion}

In conclusion, this chapter proposed a new adaptive atlas-based framework for the automated segmentation of different brain structures from DTI. The proposed segmentation technique demonstrates that the integration of a second-order MGRF spatial model with NMF-based visual appearance features is promising for controlling an adaptive shape model to segment DTI infant brains. The accuracy of the proposed segmentation framework can aid researchers to advance new methods for the detection of various brain disorders (e.g., autism and dyslexia) at an early stage. In particular, the proposed segmentation approach will be integrated into a CAD system for the early diagnosis of ASDs, where it will be used with other methodologies to explore features in the GM and WM regions of the brain (see Chapter III). 


\section{CHAPTER III}

\section{INFANT BRAIN CLASSIFICATION}

This chapter focuses on extracting discriminatory features to describe the shape and the connectivity of infant brains using diffusion tensor images. The capabilities of these extracted features in the early diagnosis of autism spectrum disorders (ASDs) are also investigated. The proposed shape analysis detects brain cortex variability using a spherical harmonic analysis that represents a 3D surface supported by the unit sphere with a linear combination of special basis functions, called spherical harmonics (SHs). The brain shape complexity is described with two shape measurements, namely, the $\mathrm{SH}$ reconstruction error and the surface complexity. The proposed connectivity analysis takes advantage of the unique information provided by diffusion tensor imaging (DTI) in providing new dimensions for the characterization of white matter (WM) anatomy. Tractography methods are applied to reconstruct WM tracts of the segmented brains, and three DTI measurements, namely, fractional anisotropy (FA), axial diffusivity $\left(\lambda_{\|}\right)$, and radial diffusivity $\left(\lambda_{\perp}\right)$ are generated and mapped to the extracted WM tracts in order to perform statistical analysis to detect differences between autistic and control brains. K-means classifier was used for each of the extracted features to evaluate their diagnostic capabilities. The preliminary diagnostic results, based on the analysis of 25 independent cases (6 autistic and 19 controls), are promising in identifying autistic from control patients. These results clearly demonstrate the promise of the proposed image-based diagnostic technique as a supplement to current diagnostic tests. 


\section{A Problem and Critical Unmet Need}

The ultimate goal of this thesis is to develop a clinically usable software tool for the accurate early diagnosis of autism through the use of diffusion tensor imaging (DTI). Informed clinical consensus defines autism spectrum disorder (ASD) as a behavioral syndrome characterized by pervasive impairment in several areas of development, including social interaction, communication skills, and other areas of interest and activity. According to the centers for disease control and prevention (CDC) report issued in 2014, one in 68 children has been diagnosed with ASDs in the United States, which is approximately 30\% greater than previous estimates reported in 2012 of one in 88 children [112]. In addition, this last report indicated that most children with ASDs are currently diagnosed after the age of four, despite the fact that ASDs can be identified as early as age two [112]. It presently costs about $\$ 3.2$ million to take care of an autistic person over his/her lifetime. The total cost of care for the autistic population within the United States is close to \$35 billion per year [181]. Therefore, developing a new technology for the early and accurate diagnosis of autism can improve the effectiveness of treatment, as well as reduce the cost of treatment. Thus far, there have been no laboratory-based measures that provide an accurate diagnosis of autism. The current gold standard autism diagnostic, as recommended by the American academy of pediatrics (AAP), is screening for developmental disabilities at 9, 18 and either 24 or 30 months. Symptomatic children are then referred to a specialist to handle the diagnosis. This diagnostic approach is not applicable at a very early age because it relies on the observation and assessment of age-dependent emergent skills in the domains of communication, social interaction, and play. Still, diagnosis is needed at early stages since early intervention is the best predictor for positive outcomes. Thus, there is an urgent need for a non-invasive technology with the capability of providing new laboratory-based measures that confer an accurate diagnosis of autism. This thesis proposes a novel computer aided diagnosis (CAD) system for the early diagno- 
sis of ASDs from DTI. The proposed CAD system consists of three main steps: (i) infant brain tissue segmentation from medical images, $(i i)$ extraction of discriminatory features (e.g. shape and connectivity features) for the segmented brain tissues, and (iii) classification of autistic from control infant brains based on analyzing the extracted features of different brain tissues for both control and autistic brains. The first step was already discussed in Chapter II, and this Chapter will cover the other two steps.

\section{B Participants}

This study included data from the Infant Brain Imaging Study (IBIS) [180], which is the parent network of an ongoing study of infants at risk for autism. The study participants are six-month-old infants with a high risk of developing ASDs. These infants were seen for follow-up assessments at 12 and 24 months of age, and the final assessment was made at age 24 months. Based on an ASD cutoff threshold, the high-risk infants were divided into two groups: ASD negative (control) and ASD-positive (autistic). From 300 subjects provided, there were only 28 subjects with available final diagnosis; however 3 subjects were excluded from further processing owing to high scan artifacts and motion distortions as indicated from the quality control report generated by the DTIprep software [152]. The final group of subjects in this study included 6 infants, which met the criteria for ASDs (4 males and 2 females), and 19 that did not (11 males and 8 females).

\section{Feature Extraction}

As demonstrated in Chapter II, DTI infant brain images were segmented into white matter (WM), grey matter (GM) and cerebrospinal fluid (CSF), which is the first step of the proposed CAD system. The next step is the extraction of discriminatory features, which are numerical values that correspond to attributes 
of the segmented structures. In particular, cortex (WM+GM) shape variability is assessed using shape construction methods. In addition to the shape information, WM integrity is further examined through connectivity analysis, where WM tracts are generated and analyzed using various DTI connectivity measurements. This section will provide more details about the feature extraction step of the proposed CAD system (see Figure 36).

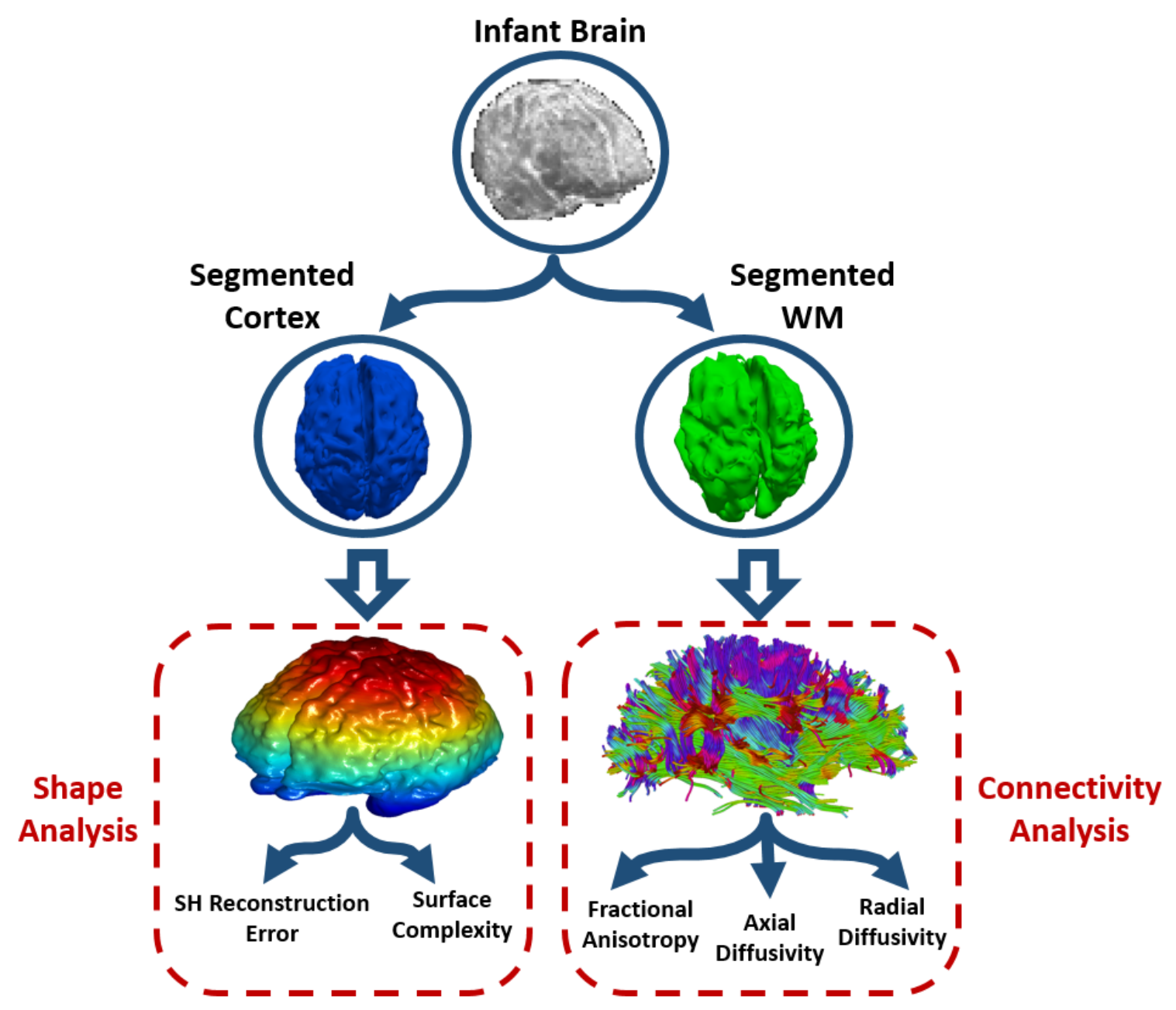

Figure 36: Illustration of the proposed feature extraction procedure. 


\section{Shape Analysis}

The shape analysis was based on spherical harmonic reconstruction [182, 183], which considers 3D surface data (i.e., brain cortex) as a linear combination of specific basis functions, namely spherical harmonics (SHs). The spherical harmonics shape analysis is performed in five steps: (i) mesh generation, (ii) mesh smoothing, (iii) unit sphere mapping, (iv) spherical harmonics reconstruction, and $(v)$ shape metrics calculation. These steps are described below.

First, a 3D mesh model of the segmented brain cortex surface is generated using a Delaunay triangulated 3D mesh [184]. Secondly, a smoothed version of the $3 \mathrm{D}$ mesh is created to ensure the uniqueness of each point in the given data set. Then, the smoothed brain mesh is mapped to a unit sphere utilizing the "Attraction-Repulsion" mapping approach [185], which dictates the mesh nodes to follow two rules: $(i)$ the unit distance of each node from the brain center, and (ii) an equal distance of each node from all of its nearest neighbors. The mapped brain cortex is approximated using a linear combination of SHs; fewer harmonics are sufficient to represent the more generic shape, while a higher-order harmonics can capture the nuanced gyrifications of the brain cortex shape. The SHs are produced by solving an isotropic heat equation for the brain cortex surface on the unit sphere [183,185]. Let $\mathcal{S}: \mathbf{M} \rightarrow \mathbf{U}$ denote the mapping of a brain mesh $\mathbf{M}$ to the unit sphere $\mathbf{U}$. Each node $\mathbf{P}=(x, y, z) \in \mathbf{M}$ mapped to the spherical position $\mathbf{u}=S(\mathbf{P})$ is represented by the spherical coordinates $\mathbf{u}=(\sin \theta \cos \varphi, \sin \theta \sin \varphi, \cos \theta)$ where $\theta \in[0, \pi]$ and $\varphi \in[0,2 \pi]$ are the polar and azimuth angles, respectively. The $\mathrm{SH}$ $Y_{\alpha \beta}$ of degree $\alpha$ and order $\beta$ is defined as [186]:

$$
Y_{\alpha \beta}=\left\{\begin{array}{lr}
c_{\alpha \beta} G_{\alpha}^{|\beta|} \cos \theta \sin (|\beta| \varphi) & -\alpha \leq \beta \leq-1 \\
\frac{c_{\alpha \beta}}{\sqrt{2}} G_{\alpha}^{|\beta|} \cos \theta & \beta=0 \\
c_{\alpha \beta} G_{\alpha}^{|\beta|} \cos \theta \cos (|\beta| \varphi) & 1 \leq \beta \leq \alpha
\end{array},\right.
$$


where $c_{\alpha \beta}=\left(\frac{2 \alpha+1}{2 \pi} \frac{(\alpha-|\beta|) !}{(\alpha+|\beta|) !}\right)^{\frac{1}{2}}$ and $G_{\alpha}^{|\beta|}$ is the associated Legendre polynomial of degree $\alpha$ and order $\beta$. For the fixed $\alpha$, the polynomials $G_{\alpha}^{\beta}$ are orthogonal over the range $[-1,1]$, as shown in [186]. The brain cortex can be simply reconstructed from the SHs of Equation (35); however, the standard least square fitting method fails to accurately model the complex shape of the brain cortex, and may result in the loss of important information that can help in the process of differentiating between autistic and normal brains. Therefore, the iterative residual fitting algorithm [187] was used to improve the approximations of the $3 \mathrm{D}$ gyrifications on the brain cortex.

Quantitative Analysis of Spherical Harmonics

To perform a quantitative analysis of the brain cortex shape, two techniques for measuring the complexity of the cerebral cortex are proposed: (i) SH reconstruction error and (ii) surface complexity.

SH reconstruction error: Due to the unit sphere mapping, the original cortex mesh for each subject is inherently aligned with the $\mathrm{SH}$ approximated mesh. The error between the original cortex mesh nodes and the $\mathrm{SH}$ approximated cortex mesh nodes can be calculated in terms of Euclidean distance. This error generates a reconstruction error curve that is unique to each subject, where the area under the curve is examined to provide a representative metric for the brain cortex [185].

Surface complexity: a new metric for examining the complexity of the brain using the $\mathrm{SH}$ coefficients is also proposed. For a unit sphere $f$, having a SH expansion as shown in Equation (35), the surface complexity metric. $S(f)$ is defined as:

$$
\begin{aligned}
S(f) & =\sum_{N=0}^{\infty} \varepsilon_{N}^{2} \\
& =\sum_{N=0}^{\infty} N B_{N}^{2},
\end{aligned}
$$

where $N$ is the number of harmonics, and $B$ are the previously calculated $\mathrm{SH}$ co- 
efficients. The squared residual $\varepsilon_{N}^{2}$ is defined as:

$$
\begin{aligned}
\varepsilon_{N}^{2} & =\left\|f-f_{N}\right\|^{2} \\
& =\left\|\sum_{n=N+1}^{\infty} \sum_{m=-n}^{n} b_{n m} Y_{n}^{m}\right\|^{2} \\
& =\sum_{n=N+1}^{\infty} \sum_{m=-n}^{n}\left|b_{n m}\right|^{2} \\
& =\sum_{n=N+1}^{\infty} B_{n}^{2}
\end{aligned}
$$

For use in 3D SH analysis there are three sets of coefficients for each direction, $\mathrm{x}, \mathrm{y}$ and $\mathrm{z}$. Therefore the surface complexity is expanded from Equation (36) to be defined as:

$$
S(f)=\frac{\sum_{N=0}^{\infty} N\left(B_{N, x}^{2}+B_{N, y}^{2}+B_{N, z}^{2}\right)}{\left\|f_{x}\right\|^{2}+\left\|f_{y}\right\|^{2}+\left\|f_{z}\right\|^{2}} .
$$

This metric generates a unique curve for each subject similar to the $\mathrm{SH}$ reconstruction error curves. Some of the advantages to this calculation are that it depends only on the SH coefficients, making it a self-contained metric, and it serves to represent the average degree of $\mathrm{SH}$ expansion. Also, it is considered as a convergent metric, and can be computed over the range of harmonics of interest.

Spherical Harmonics Analysis Results

The performance of the proposed 3D brain cortex shape analysis methods were evaluated by applying them on both the control and the autistic groups. The final goal of the proposed shape analysis is to obtain shape features that can help in distinguishing between control and autistic brains. A step-by-step of the proposed SH analysis is demonstrated in Figure 37 and Figure 38. The surface of the input segmented brain cortex (Figure 37 (a)) is approximated by a triangulated 3D mesh having 50,000 nodes (Figure $37(\mathrm{~b})$ ). Then, a smoothed version of the 3D mesh is generated (Figure 37 (c)), and mapped to a unit sphere using the "Attraction-Repulsion" algorithm [185] (Figure 37 (d)) in order to generate the final SHs to approximate the original mesh (Figure 37 (e)). Finally, the aligned original and the SHs reconstructed meshes are used to generate two shape metrics, namely, 
SH reconstruction error (Figure 38 (a)) and Surface complexity (Figure 38 (b)). Table 5 provides the complete results of the proposed two shape metrics, while some examples of SHs cortex reconstruction for different classes and genders are given in Figure 39. These preliminary results show that the proposed 3D brain cortex shape analysis methods are promising features for accurately discriminating between autistic and control subjects.

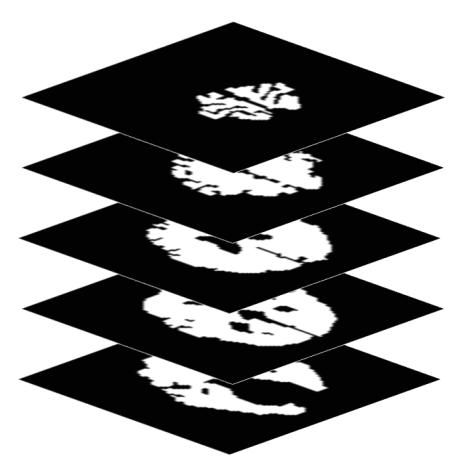

(a) Segmented brain cortex

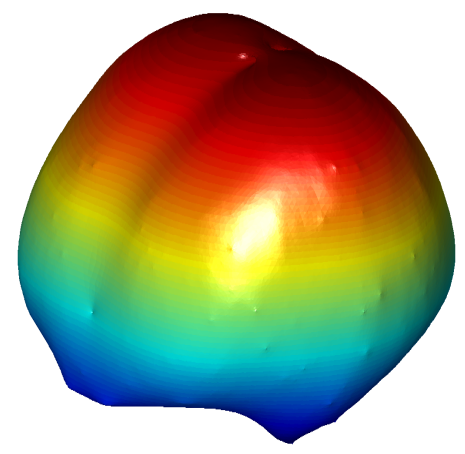

(c) Smoothed mesh

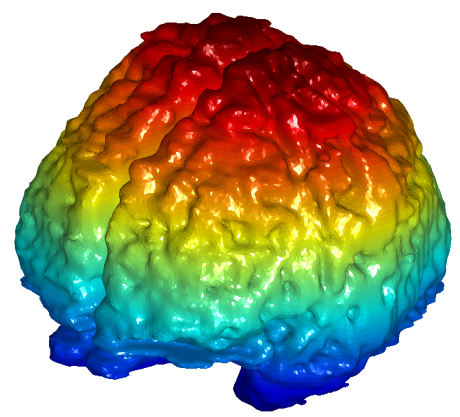

(b) Original mesh

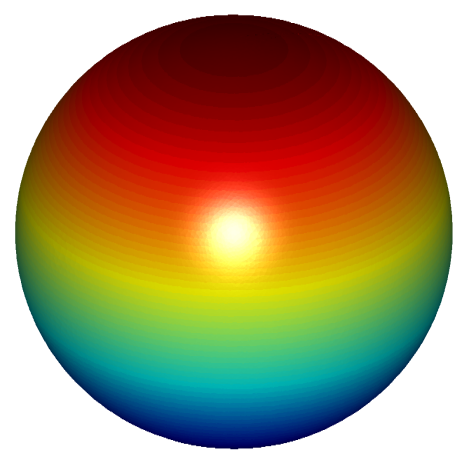

(d) Unit sphere mesh

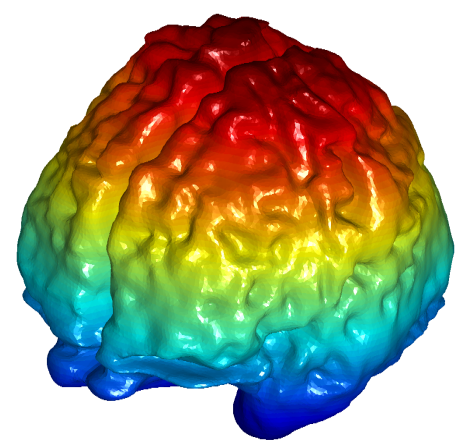

(e) SHs reconstructed mesh

Figure 37: Illustration of the SHs shape analysis steps. 


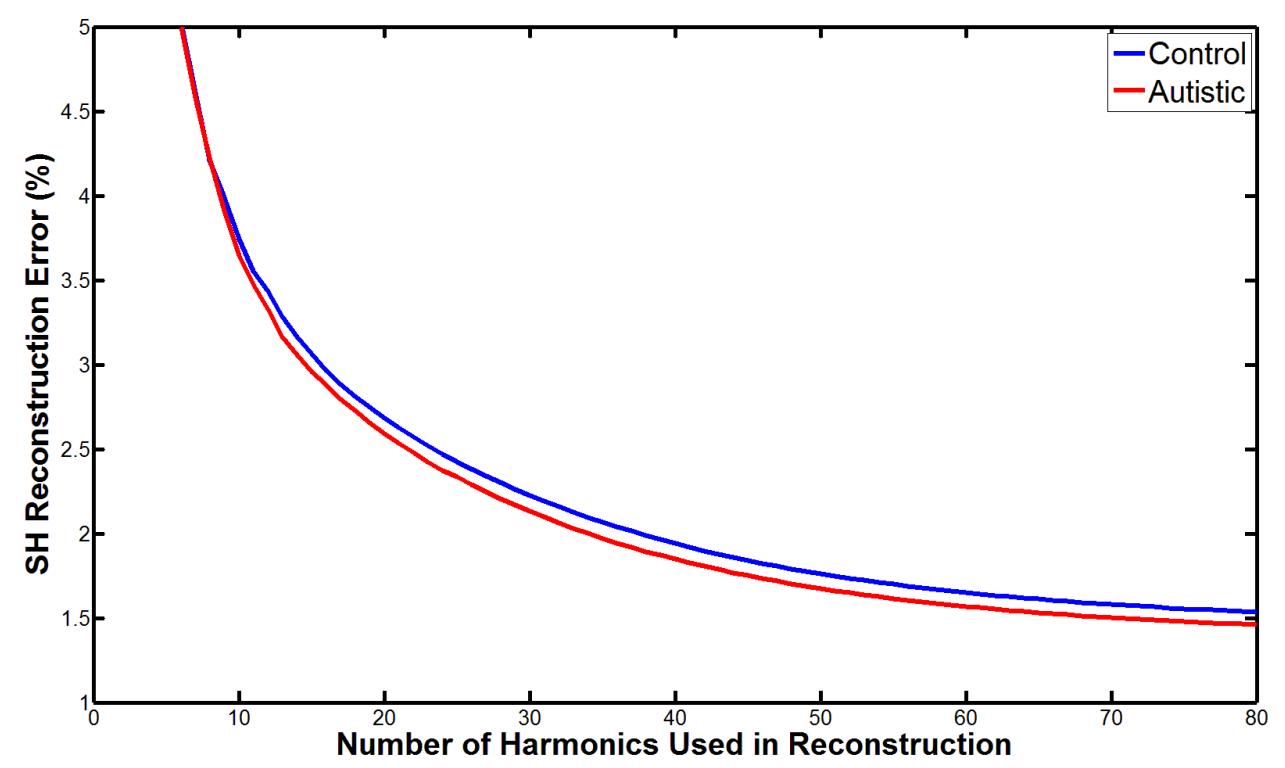

(a) $\mathrm{SH}$ reconstruction error

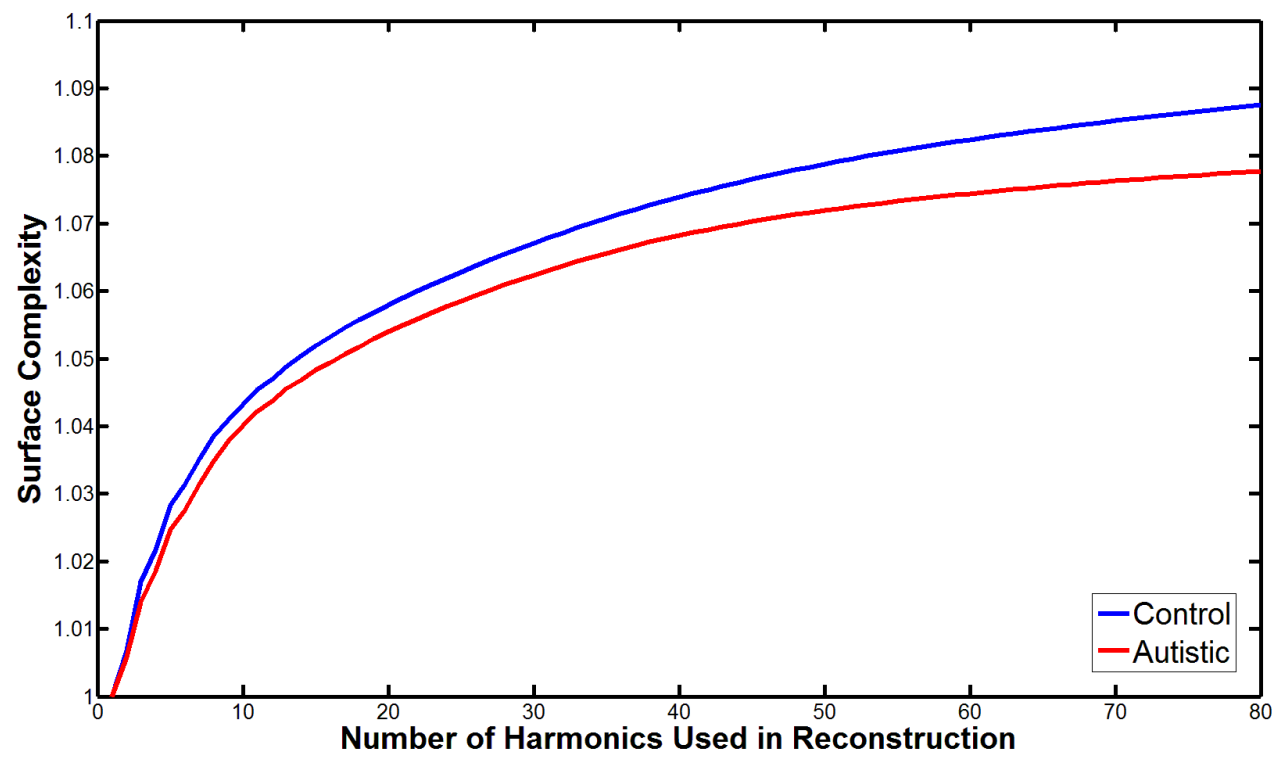

(b) Surface complexity

Figure 38: Error curves for the average autistic and control brains obtained for quantitative analysis of SHs. 
Table 5

Shape analysis results for control and autistic brains in terms of $\mathrm{SH}$ reconstruction error and Surface complexity metrics.

\begin{tabular}{|c|c|c|}
\hline Subject No. & SH reconstruction error & Complexity error \\
\hline $\mathrm{C} 1$ & 269.33 & 87.45 \\
\hline C2 & 308.54 & 91.10 \\
\hline $\mathrm{C} 3$ & 287.71 & 87.37 \\
\hline C4 & 238.40 & 84.79 \\
\hline C5 & 203.23 & 84.03 \\
\hline C6 & 253.78 & 86.28 \\
\hline $\mathrm{C} 7$ & 244.86 & 86.33 \\
\hline C8 & 204.79 & 84.97 \\
\hline C9 & 194.17 & 83.78 \\
\hline C10 & 218.06 & 85.23 \\
\hline C11 & 213.85 & 84.36 \\
\hline C12 & 245.18 & 85.31 \\
\hline $\mathrm{C} 13$ & 250.81 & 85.58 \\
\hline C14 & 201.21 & 84.34 \\
\hline C15 & 330.92 & 91.08 \\
\hline C16 & 255.32 & 85.61 \\
\hline C17 & 221.27 & 86.23 \\
\hline C18 & 248.43 & 87.77 \\
\hline C19 & 207.39 & 84.16 \\
\hline Mean \pm STD & $241.96 \pm 37.62$ & $86.09 \pm 2.12$ \\
\hline A1 & 216.82 & 84.56 \\
\hline A2 & 244.21 & 85.52 \\
\hline A3 & 187.64 & 83.81 \\
\hline A4 & 260.41 & 86.30 \\
\hline A5 & 260.01 & 87.51 \\
\hline A6 & 234.49 & 85.99 \\
\hline Mean \pm STD & $233.93 \pm 28.01$ & $85.61 \pm 1.31$ \\
\hline
\end{tabular}




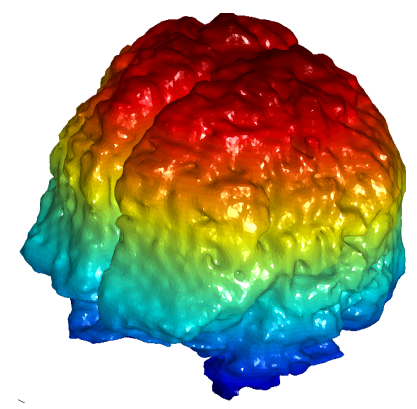

(a) Control male original mesh

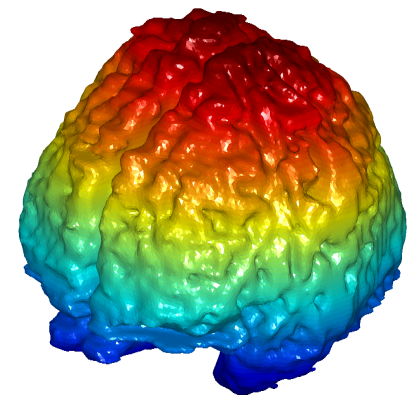

(c) Control female original mesh

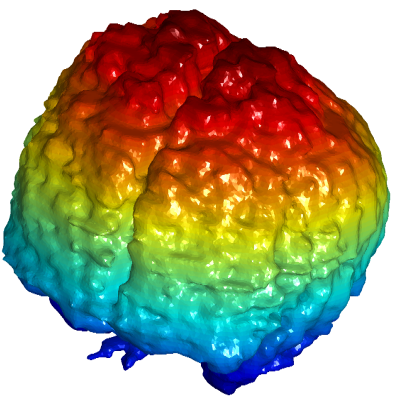

(e) Autistic male original mesh

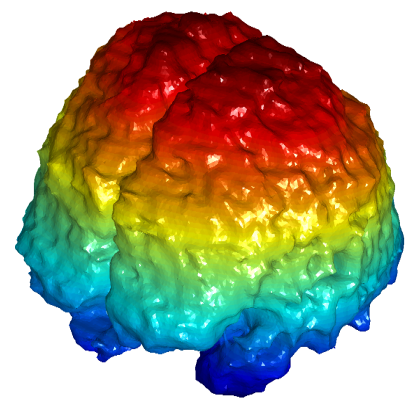

(g) Autistic female original mesh

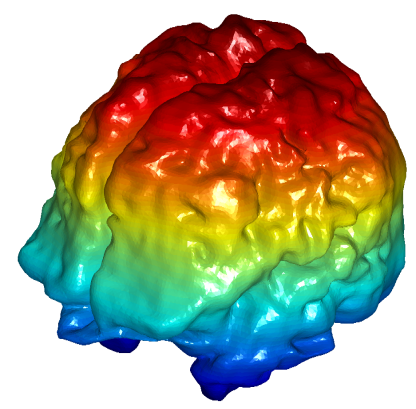

(b) Control male SH mesh

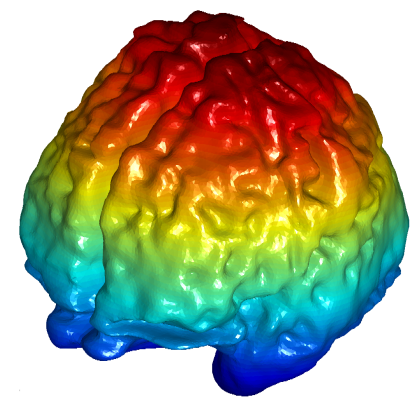

(d) Control female SH mesh

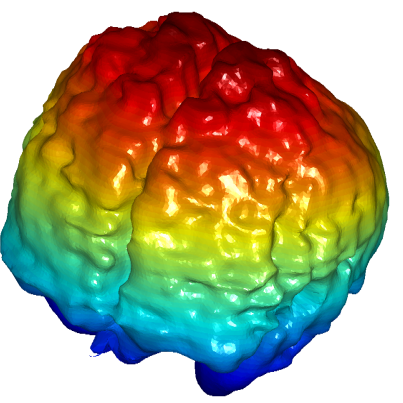

(f) Autistic male $\mathrm{SH}$ mesh

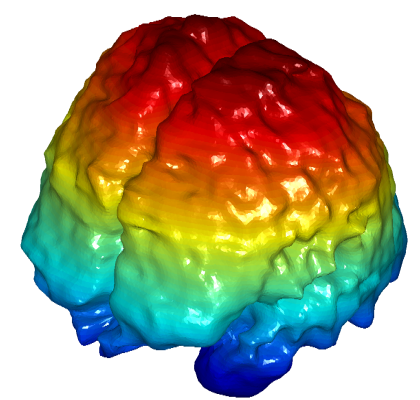

(h) Autistic female $\mathrm{SH}$ mesh

Figure 39: Examples of SH reconstructed brains compared to their original meshes. 


\section{Connectivity Analysis}

Instead of visualizing diffusion in one single voxel, tractography offers information across multiple voxels showing global connectivity of fiber bundles. Tractography analysis is proven to be very valuable in recognizing WM tracts' trajectories and their connections with other WM tracts or other GM structures. Tractography methods can be classified into two categories of tract construction techniques: (i) deterministic approaches that provide only one trajectory for each start voxel, and (ii) probabilistic approaches that use energy minimization methods to provide the path with the highest probability to link two selected voxels or regions. In this thesis, a deterministic tractography approach built in 3D slicer software [102] was used to generate the required WM fiber tracts. In this deterministic approach, the principal eigenvector $\hat{v}_{1}$ is assumed to provide a representation to the orientation of the WM fiber tract, and tractography analysis was performed in three steps: : (i) fiber orientation extraction, (ii) pathway propagation, and (iii) propagation termination [83]. First, local fiber orientation at each voxel is estimated using planar diffusion profiles or by using the full diffusion ellipsoid in the case of a highly isotropic medium. Then, the diffusion weighted images are interpolated to provide continuous voxel information, which is needed for a realistic representation of WM tracts. The next step is to propagate a single pathway, starting from a seed point, based on full tensor or principal vector information. Finally, pathway propagation is terminated when tracts enter a low anisotropy region or when a sharp bend occurs (see Chapter I for more details about WM fiber tractography).

Tractography Analysis Results

The segmented WM images, obtained in Chapter II, were used to seed tracts on the infant DTI data. Seeds were placed using $2 \mathrm{~mm}$ seed spacing, and a minimum linear measure of 0.3 for the seeding to start. For the pathway propagation 
parameters, the minimum and the maximum lengths of the fibers were set to 20 $\mathrm{mm}$ and $800 \mathrm{~mm}$, respectively. Tracking was stopped when fractional anisotropy (FA) was below 0.25 or when the radius of track curvature becomes smaller than $0.7^{\circ} / \mathrm{mm}$. After WM fiber tracts were extracted, FA values were generated for each fiber tract to measure the degree of anisotropy of local diffusivity. In addition to FA values, axial $\left(\lambda_{\|}\right)$and radial $\left(\lambda_{\perp}\right)$ diffusivity values, which represent diffusion parallel and transverse to axonal directions, were also produced (see Chapter I for more details about these three DTI measurements). An example of the generated WM fiber tracts is shown in Figure 40. Table 6 provides the mean values of the three generated DTI measurements across all the extracted WM tracts, and Figure 41 provides a boxplot representation of the results in both control and autistic groups. These preliminary results show relatively higher values of all the generated DTI measurements in the autistic brains, when compared to the control brains. These initial results indicates that the extracted DTI features are promising in differentiating between autistic and control subjects.

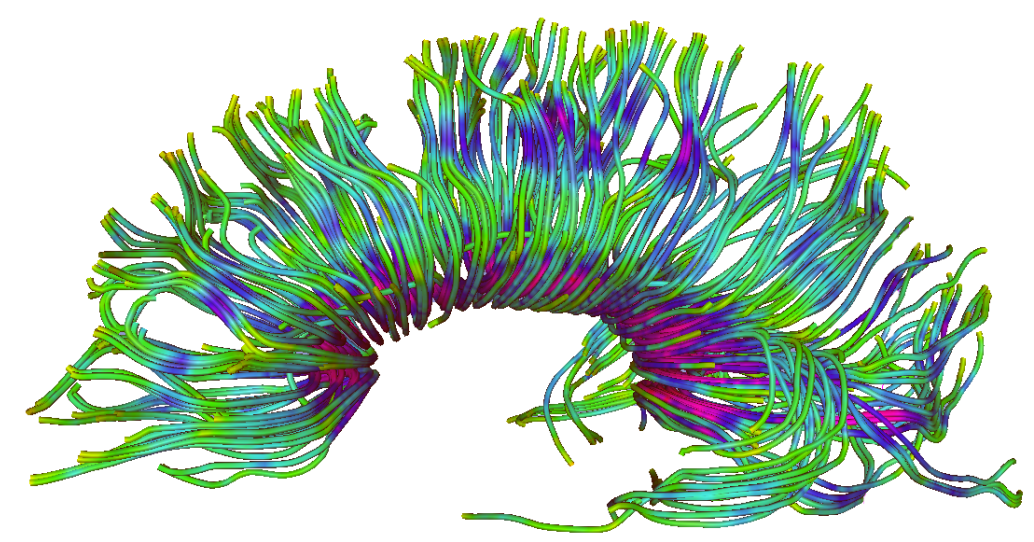

Figure 40: Example of the extracted white matter fiber tracts, generated within the corpus callosum region, where colors have been assigned based on the FA values along the tracts. 
Table 6

Connectivity analysis results for control and autistic brains in terms of the mean values of fractional anisotropy, axial diffusivity, and radial diffusivity in the extracted WM fiber tracts.

\begin{tabular}{|c|c|c|c|}
\hline \hline Subject No. & Fractional Anisotropy & Axial Diffusivity & Radial Diffusivity \\
\hline C1 & 0.692 & 4.497 & 0.916 \\
\hline C2 & 0.610 & 3.195 & 0.995 \\
\hline C3 & 0.723 & 4.774 & 1.457 \\
\hline C4 & 0.709 & 3.182 & 0.804 \\
\hline C5 & 0.634 & 4.325 & 1.500 \\
\hline C6 & 0.566 & 2.201 & 0.818 \\
\hline C7 & 0.650 & 3.726 & 0.940 \\
\hline C8 & 0.604 & 2.522 & 0.883 \\
\hline C9 & 0.656 & 5.217 & 1.715 \\
\hline C10 & 0.582 & 2.653 & 0.901 \\
\hline C11 & 0.704 & 3.748 & 0.920 \\
\hline C12 & 0.637 & 8.808 & 3.144 \\
\hline C13 & 0.889 & 20.010 & 1.539 \\
\hline C14 & 0.748 & 4.480 & 0.864 \\
\hline C15 & 0.664 & 3.025 & 0.898 \\
\hline C16 & 0.629 & 3.238 & 0.905 \\
\hline C17 & 0.602 & 2.791 & 0.873 \\
\hline C18 & 0.763 & 4.343 & 0.843 \\
\hline C19 & 0.712 & 4.663 & 1.454 \\
\hline Mean $\pm S T D$ & $0.672 \pm 0.077$ & $4.810 \pm 3.957$ & $1.177 \pm 0.561$ \\
\hline A1 & 0.653 & 3.110 & 0.797 \\
\hline A2 & 0.716 & 6.897 & 2.157 \\
\hline A3 & 0.707 & 3.053 & 0.836 \\
\hline A4 & 0.685 & 3.734 & 0.961 \\
\hline A5 & 0.711 & 40.866 & 3.484 \\
\hline A6 & 0.669 & $5.290 \pm 3.076$ & $1.549 \pm 1.074$ \\
\hline Mean $\pm S T D$ & $0.690 \pm 0.025$ & & \\
\hline & & & \\
\hline & & & \\
\hline
\end{tabular}




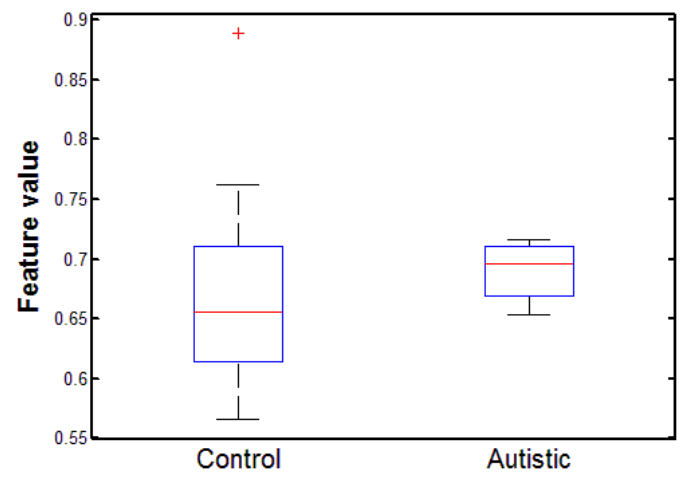

(a) Fractional anisotropy

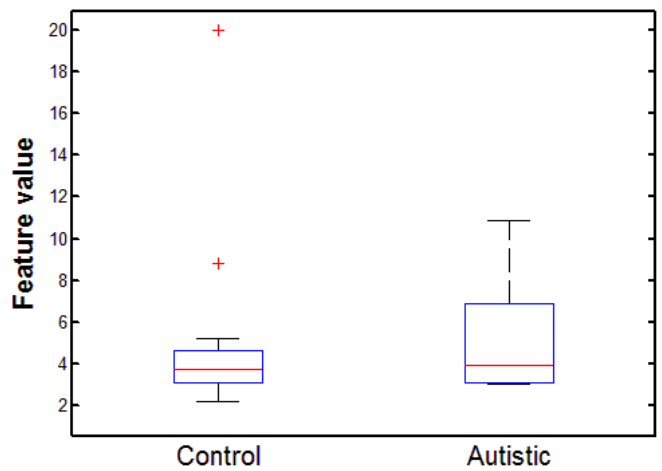

(b) Axial diffusivity

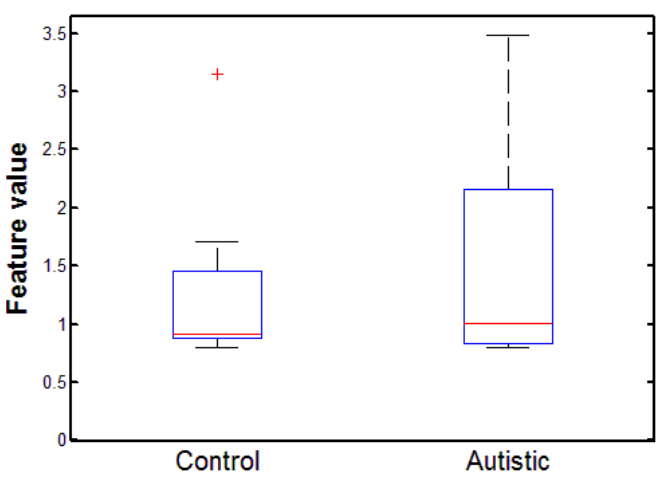

(c) Radial diffusivity

Figure 41: Boxplots representing the mean values of fractional anisotropy, axial diffusivity, and radial diffusivity in the extracted WM fiber tracts for both control and autistic brains. 


\section{Classification}

Based on the five features extracted from the infant DTI data, the potential of each feature is tested to identify autistic and control subjects. To perform this task, five k-means classifiers were built, using each feature, to evaluate the diagnostic capability of each feature. For each classifier, the data is divided into control (19 subjects) and autistic (6 subjects) groups and the mean of each feature in each groups is calculated. Each subject is classified as autistic or control subject based on the closest Euclidian distance to the two group means. Table 7 summarizes the classification results using each feature.

Table 7

Classification results using the five features that are extracted from the analysis of the DTI data. A classifier is built for each feature and the results are given in terms of the accuracy.

\begin{tabular}{|c|c|c|c|}
\hline \hline Feature & Control & Autistic & Overall \\
\hline SH Reconstruction Error & $\begin{array}{c}57.895 \% \\
(11 \text { out of } 19)\end{array}$ & $\begin{array}{c}50 \% \\
(3 \text { out of } 6)\end{array}$ & $\begin{array}{c}56 \% \\
(14 \text { out of } 25)\end{array}$ \\
\hline Surface Complexity & $\begin{array}{c}42.105 \% \\
\text { (8 out of } 19)\end{array}$ & $\begin{array}{c}50 \% \\
(3 \text { out of } 6)\end{array}$ & $\begin{array}{c}44 \% \\
(11 \text { out of } 25)\end{array}$ \\
\hline Mean Fractional Anisotropy & $57.895 \%$ & $66.667 \%$ & $60 \%$ \\
& $(11$ out of 19$)$ & $(4$ out of 6$)$ & $(15$ out of 25$)$ \\
\hline Mean Axial Diffusivity & $84.211 \%$ & $33.333 \%$ & $72 \%$ \\
& $(16$ out of 19$)$ & $(2$ out of 6$)$ & $(18$ out of 25$)$ \\
\hline Mean Radial Diffusivity & $\begin{array}{c}68.421 \% \\
(13 \text { out of } 19)\end{array}$ & $\begin{array}{c}33.333 \% \\
(2 \text { out of } 6)\end{array}$ & $\begin{array}{c}60 \% \\
(15 \text { out of } 25)\end{array}$ \\
\hline
\end{tabular}

As shown in the table, the preliminary results based on the available data sets ( 25 subjects: 6 autistic and 19 control) show that some features are outstanding candidates to distinguish between the autistic and control groups. For example, the mean FA shows a potential to identify the autistic subjects with an accuracy of $67 \%$, where as the mean axial diffusivity shows a potential to identify the control subjects with an accuracy of $84 \%$. These results are encouraging to to be extended. 
Since the available datasets are limited (only 6 autistic subjects), more data are planned to be collected to build more powerful classifiers. In addition, our future work include to fuse between five features and use the extended collected dataset to design a more powerful classifier that integrates all five DTI features, based on the developed genetic algorithm proposed by Khalifa et al. [188]. 


\section{CHAPTER IV}

\section{CONCLUSION AND FUTURE WORK}

This thesis proposes a novel computer aided diagnosis (CAD) system for the early diagnosis of autism spectrum disorders (ASDs) from diffusion tensor imaging (DTI). The proposed CAD system integrates both shape and connectivity extracted features in the classification process. This CAD system consists of three main steps: (i) infant brain tissue segmentation from medical images, (ii) extraction of discriminatory features (e.g. shape and connectivity features) for the segmented brain tissues, and (iii) classification of autistic from control infant brains based on analyzing the extracted features of different brain tissues for both control and autistic brains. The proposed models and techniques developed in this thesis show promising results for a variety of medical applications:

- Modeling the shape of complex medical structures such as white matter (WM), grey matter (GM), and cerebrospinal fluid (CSF) $[189,190]$.

- Modeling the visual appearance of a large dimensional feature space by extracting new meaningful features with reduced dimensionality and increased separation ability.

- Automatic segmentation of the brain from DTI into corresponding tissues (i.e., WM, GM, and CSF) $[189,190]$.

- Deriving efficient quantitative geometrical features to describe the complexity of the infant brain cortex shape.

- Extracting DTI-based features to asses the connectivity of WM fiber tracts. 
- Developing a CAD system using DTI for early diagnosis of ASDs.

In the following section, a summary of the most important contributions in this thesis is presented.

\section{A Contributions}

- This thesis proposes a novel infant brain extraction approach to automatically remove any non-brain tissues (e.g., eyes, dura, and skull) from the input diffusion weighted images. The proposed approach is based on a first-order visual appearance model that guides an edge-preserving image restoration model to account for intensity inhomogeneity through an energy minimization optimization procedure. The proposed approach has been evaluated using three performance metrics: the Dice similarity coefficient (DSC), 95percentile modified Hausdorff distance $\left(\mathrm{H}_{95}\right)$, and the absolute volume difference (AVD) [179]. As demonstrated in the experimental results, the DSC for extracting the brain is $96.64 \pm 1.15 \%$, which confirms the high accuracy of the proposed brain extraction approach.

- This thesis proposes a novel atlas-based infant brain segmentation framework $[189,190]$ for the automated segmentation of different brain structures (e.g., CSF, GM, and WM) from infant diffusion tensor images in the isointense age stage (6-12 months). The proposed framework relies on a joint Markov-Gibbs random field (MGRF) model that accounts for three image descriptors: a novel nonnegative matrix factorization (NMF) based visual appearance model, a novel adaptive shape model, and a 3D spatially invariant second-order MGRF homogeneity descriptor. The performance of the proposed segmentation framework has been evaluated using three performance metrics: DSC, $\mathrm{H}_{95}$, and AVD [179]. The DSC for segmentation results of the WM, GM, and the CSF are $95.23 \pm 1.18 \%, 89.92 \pm 2.86 \%$, and $87.96 \pm 3.31 \%$, re- 
spectively, which demonstrates that the integration of a second-order MGRF spatial model with NMF-based visual appearance features is promising for controlling an adaptive shape model to segment DTI infant brains. The accuracy of the proposed segmentation framework can aid researchers to advance new methods for the detection of various brain disorders (e.g., autism and dyslexia) at an early stage.

- This thesis presents a novel NMF-based visual appearance model that has the ability to model a large dimensional feature space by extracting new meaningful features with reduced dimensionality and increased separation ability. The NMF-based visual appearance descriptor is modeled using a Kmeans classifier with centroids calculated in the training phase. The introduction of the new NMF-fused features showed promising success in terms of segmentation accuracy and speed in the case of a large input feature space.

- This thesis presents a novel adaptive shape model [190] that has the ability to capture the shape variation of complex brain structures, such as WM, GM, and CSF, by accounting for the first- and second-order visual appearance characteristics of new NMF-fused image features of the medical structures. The adaptive shape model has been successfully used to guide the classification of brain tissue and has shown an ability to account for the complexity of the brain structures [189]. The preliminary results of this model confirm its benefits and encourage using it to model other medical structures.

- This thesis presents a novel framework for diagnosing autism using diffusion tensor infant brain images. The proposed framework relied on $\mathrm{SH}$ shape analysis to produce two novel shape measurements to describe the brain shape complexity, and a tractography-based connectivity analysis to assess the integrity of the brain WM tracts using three different DTI-based measurements. The preliminary diagnostic results, based on the available 
data sets, are promising in identifying autistic from control patients.

\section{B Future Work}

The work presented in this thesis can be further enhanced and extended as follows:

- The proposed brain segmentation framework will be extended to include the use of advanced DTI features that can reflect new information about various brain structures. Also, the optimum level of dimensionality reduction used in the NMF-based feature fusion process will be investigated. To verify the robustness of the proposed segmentation framework, it will be tested with a larger data set and compared with the current infant brain segmentation techniques.

- The proposed brain segmentation framework has been used to segment WM, GM, and CSF brain structures. Future work includes investigating the ability of the proposed method to segment other brain structures, such as the corpus callosum, the hippocampus, and the cerebellum.

- The proposed SHs-based shape analysis framework relied on two quantitative techniques for measuring the complexity of the cerebral cortex. In the future, additional features derived from SHs will be investigated, and additional brain structures (e.g., cerebral WM [191] and corpus callosum [192]) will be explored in order to quantitatively characterize brain changes associated with autistic brains.

- The proposed brain classification framework will be extended to involve advanced identification of brain regions that have significant differences between autistic and control subjects using constructed brain maps. 
- The proposed CAD system for early diagnosis of ASDs will be tested on larger data sets with known ground truths (i.e., the doctors' diagnoses), and will be used to explore longitudinal scans to study the temporal development of different brain structures and track changes in the brain that are attributed to ASDs.

- The ultimate goal of the work proposed in this thesis is to develop a CAD system for early diagnosis of ASDs. A future extension of this work would be analyzing the extracted brain and testing these measurements not only to diagnose autism [191-198], but also to characterize physiological processes and other disease entities or to characterize the severity of other diseases such as dyslexia [199, 200], attention deficit disorder (ADD), brain tumors, strokes, seizure disorders, depression, and Alzheimer's disease [201].

- Another future direction is applying the developed models in other clinical applications such as: acute renal rejection [202-211], lung cancer detection [212-237, 237-245], and cancerous cells detection in the prostate [246-252]. 


\section{REFERENCES}

[1] P. J. Basser, J. Mattiello, and D. LeBihan. Estimation of the effective selfdiffusion tensor from the NMR spin echo. Journal of Magnetic Resonance, Series $B, 103(3): 247-254,1994$.

[2] P. J. Basser, J. Mattiello, and D. LeBihan. Mr diffusion tensor spectroscopy and imaging. Biophysical journal, 66(1):259-267, 1994.

[3] Q. Dong, R. C. Welsh, T. L. Chenevert, R. C. Carlos, P. Maly-Sundgren, D. M. Gomez-Hassan, and S. K. Mukherji. Clinical applications of diffusion tensor imaging. Journal of Magnetic Resonance Imaging, 19(1):6-18, 2004.

[4] G. Dawson. Early behavioral intervention, brain plasticity, and the prevention of autism spectrum disorder. Development and psychopathology, 20(03):775-803, 2008.

[5] N. A. Obuchowski, R. J. Graham, M. E. Baker, and K. A. Powell. Ten criteria for effective screening: their application to multislice ct screening for pulmonary and colorectal cancers. American Journal of Roentgenology, 176(6):1357-1362, 2001.

[6] B. Lee and A. Newberg. Neuroimaging in traumatic brain imaging. The Journal of the American Society for Experimental NeuroTherapeutics, 2(2):372383, 2005.

[7] A. El-Baz, G. Gimelfarb, R. Falk, and M. A. El-Ghar. Automatic analysis of 3D low dose CT images for early diagnosis of lung cancer. Pattern Recognition Journal, 42(2):1041-1051, 2009. 
[8] S. C. Park, J. Tan, X. Wang, D. Lederman, J. K. Leader, S. H. Kim, , and B. Zheng. Computer-aided detection of early interstitial lung diseases using low-dose CT images. Physiscs in Medicine and Biology, 56(4):1139-1153, 2011.

[9] P. Ou, D. S. Celermajer, G. Calcagni, F. Brunelle, D. Bonnet, and D. Sidi. Three-dimensional ct scanning: a new diagnostic modality in congenital heart disease. Heart, 93(8):908-913, 2007.

[10] S. Sheth and E. K. Fishman. Multi-detector row CT of the kidneys and urinary tract: Techniques and applications in the diagnosis of benign diseases. Radiographics, 24(2):e20, 2004.

[11] A. Jhamb, R. S. Dolas, P. K. P., and S. Mohanty. Comparative efficacy of spiral computed tomography and orthopantomography in preoperative detection of relation of inferior alveolar neurovascular bundle to the impacted mandibular third molar. Journal of Oral and Maxillofacial Surgery, 67(1):58-66, 2009.

[12] S. Bisdasand, G. Konstantinou, K. Surlan-Popovic, A. K. M., Baghi, T. J. Vogl, T. S. Koh, and M. G. Mack. Dynamic contrast-enhanced CT of head and neck tumors: comparison of first-pass and permeability perfusion measurements using two different commercially available tracer kinetics models. Academic Radiology, 15(12):1580-1589, 2008.

[13] S. J. Swensen. Functional CT: Lung nodule evaluation1. RadioGraphics, 20(1):1178-1181, 2000.

[14] J. G. Korporaal, C. A. T. van den Berg, C. R. L. P. N. Jeukens, G. Groenendaal, M. R. Moman, P. Luijten, M. van Vulpen, and U. A. van der Heide. Dynamic contrast-enhanced CT for prostate cancer: Relationship between image noise, voxel size, and repeatability. Radiology, 256(3):976-984, 2010. 
[15] K. A. Miles. Functional computed tomography in oncology. European Journal of Cancer, 38(16):2079-2084, 2002.

[16] P. D. Stein, A. Y. Yaekoub, F. Matta, and H. D. Sostman. Sixty-four-slice CT for diagnosis of coronary artery disease: a systematic review. The American Journal of Medicine, 121(8):715-725, 2008.

[17] P. Verro, L. N. Tanenbaum, N. Borden, N. Eshkar, and S. Sen. Clinical application of ct angiography in acute ischemic stroke. Clinical Neurology and Neurosurgery, 109(2):138-145, 2007.

[18] F. Fraioli, C. Catalano, L. Bertoletti, M. Danti, F. Fanelli, A. N. M. Cavacece, and R. Passariello. Multidetector-row CT angiography of renal artery stenosis in 50 consecutive patients: prospective interobserver comparison with DSA. Radiologia Medica, 111(2):459-468, 2006.

[19] M. Dewailly, M. Rémy-Jardin, A. Duhamel, J. B. Faivre, F. Pontana, V. Deken, A. M. Bakai, and J. Remy. Computer-aided detection of acute pulmonary embolism with 64-slice multi-detector row computed tomography: Impact of the scanning conditions and overall image quality in the detection of peripheral clots. Journal of Computer Assisted Tomography, 34(1):23-30, 2010.

[20] D. W. Holdsworth and M. M. Thornton. Micro-CT in small animal and specimen imaging. Trends in Biotechnology, 8(1):34-39, 2002.

[21] J. J. Vaquero, S. Redondo, E. Lage, M. Abella, A. Sisniega, G. Tapias, M. L. S. Montenegro, and M. Desco. Assessment of a new high-performance smallanimal X-ray tomography. IEEE Transactions on Nuclear Science, 55(3):898905, 2008.

[22] F. Neues and M. Epple. X-ray microcomputer tomography for the study of biomineralized endo- and exoskeletons of animals. Chemical Reviews, 108(11):4734-4741, 2008. 
[23] M. E. Oest, J. C. Jones, C. Hatfield, and M. R. Prater. Micro-CT evaluation of murine fetal skeletal development yields greater morphometric precision over traditional clear-staining methods. Birth Defects Research Part B: Developmental and Reproductive Toxicology, 83(6):582-589, 2008.

[24] K. A. Johnson. Imaging techniques for small animal imaging models of pulmonary disease: Micro-CT. Toxicologic Pathology, 35(1):59-64, 2007.

[25] B. A. Kerr and T. V. Byzova. MicroCT: An essential tool in bone metastasis research. In L. Saba, editor, Computed Tomography-Clinical Applications, volume 1, chapter 13, pages 211-230. 2012.

[26] C. T. Badea, L. W. Hedlund, J. Cook, B. R. Berridge, and G. A. Johnson. MicroCT imaging assessment of dobutamine-induced cardiac stress in rats. Journal of Pharmacological and Toxicological Methods, 63(1):24-29, 2011.

[27] D. O. Cosgrove. Manual of diagnostic ultrasound, volume 1, 2012.

[28] A. G. Webb. Introduction to Biomedical Imaging. Wiley-IEEE Press, NJ, USA.

[29] T. A. Whittingham. Medical diagnostic applications and sources. Progress in Biophysics and Molecular Biology, 93(1-3):84-110, 2007.

[30] S. M. Bunce, A. D. Hough, and A. P. Moore. Measurement of abdominal muscle thickness using M-mode ultrasound imaging during functional activities. Manual Therapy, 9:41-44, 2004.

[31] J. T. Salonen and R. Salonen. Ultrasound B-mode imaging in observational studies of atherosclerotic progression. Circulation, 87(3):56-65, 1993.

[32] R. Kern, K. Szabo, M. Hennerici, and S. Meairs. Characterization of carotid artery plaques using real-time compound B-mode ultrasound. Stroke, 35:870-875, 2004. 
[33] R. S. Lazebnik and T. S. Desser. Clinical 3D ultrasound imaging: beyond obstetrical applications. Diagnostic Imaging, pages 1-5, 2007.

[34] S. R. Wilson, L. D. Greenbaum, and B. B. Goldberg. Contrast-enhanced ultrasound: What is the evidence and what are the obstacles?. American Journal of Roentgenology, 193(1):55-60, 2009.

[35] N. B. Smith and A. Webb. Introduction to medical imaging: Physics, engineering and clinical applications. Cambridge University Press, 2010.

[36] P. Mansfield. Snapshot magnetic resonance imaging (nobel lecture). Angewandte Chemie International Edition, 43(41):5456-5464, 2004.

[37] E. M. Haacke. Magnetic Resonance Imaging: Physical Principles and Sequence Design. J. Wiley \& Sons, New York, USA, 1999.

[38] F. W. Wehrli, J. MacFall, T. H. Newton, and D. G. Potts. Advanced Imaging Techniques. Clavadel Press, San Francisco, CA, USA, 1983.

[39] W. G. Bradley. Effect of magnetic relaxation times on magnetic resonance image interpretation. Noninvasive Medical Imaging, 1:193-204, 1984.

[40] S. Ogawa, T. M. Lee, A. S. Nayak, and P. Glynn. Oxygenation-sensitive contrast in magnetic resonance image of rodent brain at high magnetic fields. Magnetic Resonance in Medicine, 14(1):68-78, 1990.

[41] A. Carpentier, K. R. Pugh, M. Westerveld, C. Studholme, O. Skrinjar, J. L. Thompson, D. D. Spencer, and R. T. Constable. Functional MRI of language processing: dependence on input modality and temporal lobe epilepsy. Epilepsia, 42(10):1241-1254, 2001.

[42] C. S. Carter, A. W. M. III, and L. L. R. V. A. Stenger. Anterior cingulate cortex activity in impaired self-monitoring of performance in patients with 
schizophrenia: An event-related fMRI study. American Journal of Psychiatry, 158(10):1423-1428, 2001.

[43] W. R. Staines, W. E. Mcilroy, S. J. Graham, and S. E. Black. Bilateral movement enhances ipsilesional cortical activity in acute stroke: A pilot functional MRI. Neurology, 56(3):401-404, 2001.

[44] H. Michaely, K. Herrmann, K. Nael, N. Oesingmann, M. Reiser, and S. Schoenberg. Functional renal imaging: Nonvascular renal disease. Abdominal Imaging, 32(1):1-16, 2007.

[45] A. Jackson, J. P. O'Connor, G. J. Parker, and G. C. Jayson. Imaging tumor vascular heterogeneity and angiogenesis using dynamic contrast-enhanced magnetic resonance imaging. Clinical Cancer Research, 13(12):3449-3459, 2007.

[46] F. Khalifa, G. M. Beache, G. Gimel'farb, and A. El-Baz. A novel CAD system for analyzing cardiac first-pass MRI images. In Proceedings International Conference on Pattern Recognition, (ICPR'12), pages 77-80, Tsukuba Science City, Japan, November 11-15, 2012.

[47] A. El-Baz, A. Farag, R. Fahmi, S. Yuksel, M. A. El-Ghar, and T. Eldiasty. Image analysis of renal DCE MRI for the detection of acute renal rejection. In Proceedings International Conference on Pattern Recognition, (ICPR'06), pages 822-825, Hong Kong, August 20-24, 2006.

[48] A. Farag, A. El-Baz, S. Yuksel, M. A. El-Ghar, and T. Eldiasty. A framework for the detection of acute rejection with dynamic contrast enhanced magnetic resonance imaging. In Proceedings IEEE International Symposium on Biomedical Imaging: From Nano to Macro, (ISBI'06), pages 418-421, Arlington, Virginia, USA, April 69, 2006. 
[49] A. El-Baz, A. A. Farag, S. E. Yuksel, M. E. A. El-Ghar, T. A. Eldiasty, and M. A. Ghoneim. Application of deformable models for the detection of acute renal rejection. In A. A. Farag and J. S. Suri, editors, Deformable Models, volume 1, chapter 10, pages 293-333. 2007.

[50] A. El-Baz, G. Gimel'farb, and M. A. El-Ghar. New motion correction models for automatic identification of renal transplant rejection. In Proceedings International Conference Medical Image Computing and Computer-Assisted Intervention, (MICCAI'07), pages 235-243, Brisbane, Australia, October 29 - November 2, 2007.

[51] A. El-Baz, G. Gimel'farb, and M. A. El-Ghar. A novel image analysis approach for accurate identification of acute renal rejection. In Proceedings IEEE International Conference on Image Processing, (ICIP'08), pages 1812-1815, San Diego, California, USA, October 12-15, 2008.

[52] A. El-Baz, G. Gimel'farb, and M. A. El-Ghar. Image analysis approach for identification of renal transplant rejection. In Proceedings International Conference on Pattern Recognition, ICPR-2008, pages 1-4, Tampa, Florida, USA, December 8-11, 2008.

[53] F. Khalifa, A. El-Baz, G. Gimel'farb, and M. A. El-Ghar. Non-invasive imagebased approach for early detection of acute renal rejection. In Proceedings International Conference Medical Image Computing and Computer-Assisted Intervention, (MICCAI'10), pages 10-18, Beijing, China, September 20-24, 2010.

[54] A. Firjani, F. Khalifa, A. Elnakib, G. Gimel'farb, A. Elmaghraby, and A. ElBaz. A novel image-based approach for early detection of prostate cancer. In Proceedings IEEE International Conference on Image Processing, (ICIP'12), pages 2849-2852, Lake Buena Vista, Florida, USA, 30 September- 3 October, 2012. 
[55] A. Firjani, F. Khalifa, A. Elnakib, G. Gimel'farb, M. A. El-Ghar, A. Elmaghraby, and A. El-Baz. Non-invasive image-based approach for early detection of prostate cancer. In Proceedings Fourth International Conference on Developments in eSystems Engineering, DeSE-11, pages 172-177, Dubai, UAE, December 6-8, 2011.

[56] J. Jackson, D. J. Allison, and J. Meaney. Angiography: principles, techniques, and complications. In R. C. Grainger, D. Allison, A. Adam, and A. K. Dixon, editors, Diagnostic Radiology: A Textbook of Medical Imaging, chapter 6. Churchill Livingstone, New York, NY, 2008.

[57] E. L. Barbier, L. Lamalle, and M. Décorps. Methodology of brain perfusion imaging. J. Magn. Reson. Imaging, 13(4):496-520, 2001.

[58] J. Lu, K. Li, M. Zhang, and L. Jiao. Dynamic susceptibility contrast perfusion magnetic resonance imaging in patients with symptomatic unilateral middle cerebral artery stenosis or occlusion. Acta Radiology, 48(3):335-340, 2007.

[59] D. D. M. Lin, J. T. Kleinman, R. J. Wityk, R. F. Gottesman, A. E. Hillis, A. Lee, and P. B. Barker. Crossed cerebellar diaschisis in acute stroke detected by dynamic susceptibility contrast MR perfusion imaging. American Journal of Neuroradiology, 30(4):710-715, 2009.

[60] E. S. Paulson and K. M. Schmainda. Comparison of dynamic susceptibilityweighted contrast-enhanced MR Methods: Recommendations for measuring relative cerebral blood volume in brain tumors. Radiology, 249(2):601$613,2008$.

[61] N. Shah, A. Sattar, M. Benanti, S. Hollander, and L. Cheuck. Magnetic resonance spectroscopy as an imaging tool for cancer: A review of the literature. Journal of the American Osteopathic Association, 106(1):23-27, 2006. 
[62] A. Horská, P. B. Barker, and D. Phil. Imaging of brain tumors: MR spectroscopy and metabolic imaging. Neuroimaging Clinics of North America, 20(3):293-310, 2010.

[63] P. Swindle, S. McCredie, P. Russell, U. Himmelreich, M. Khadra, C. Lean, and C. Mountford. Pathologic characterization of human prostate tissue with proton MR spectroscopy. Radiology, 228(1):144-151, 2003.

[64] L. L. Cheng, M. A. Burns, J. L. Taylor, W. He, E. F. Halpern, W. S. McDougal, and C. L. Wu. Metabolic characterization of human prostate cancer with tissue magnetic resonance spectroscopy. Cancer Research, 65(8):3030-3034, 2005.

[65] M. A. Jacobs, P. B. Barker, P. Argani, R. Ouwerkerk, Z. M. Bhujwalla, and D. A. Bluemke. Combined dynamic contrast enhanced breast MR and proton spectroscopic imaging: A feasibility study. Journal of Magnetic Resonance Imaging, 21(1):23-28, 2005.

[66] M. A. Jacobs, P. B. Barker, P. A. Bottomley, Z. Bhujwalla, and D. A. Bluemke. Proton magnetic resonance spectroscopy imaging of human breast cancer: a preliminary study. Journal of Magnetic Resonance Imaging, 19(1):68-75, 2004.

[67] M. M. Mahon, I. J. Cox, R. Dina, W. P. Soutter, G. A. McIndoe, A. D. Williams, and N. M. deSouza. ${ }^{1} \mathrm{H}$ magnetic resonance spectroscopy of preinvasive and invasive cervical cancer: in vivo-ex vivo profiles and effect of tumor load. Journal of Magnetic Resonance Imaging, 19(1):256-364, 2004.

[68] M. M. Mahon, A. D. Williams, W. P. Soutter, I. J. Cox, G. A. McIndoe, G. A. Coutts, R. Dina, and N. M. deSouza. ${ }^{1} \mathrm{H}$ magnetic resonance spectroscopy of invasive cervical cancer: an in vivo study with ex vivo corroboration. NMR in Biomedicine, 17(1):1-9, 2004. 
[69] S. G. Cho, D. H. Lee, K. Y. Lee, H. Ji, K. H. Lee, P. R. Ros, and C. H. Suh. Differentiation of chronic focal pancreatitis from pancreatic carcinoma by in vivo proton magnetic resonance spectroscopy. Journal Computer Assisted Tomography, 29(2):163-169, 2005.

[70] S. T. Doran, G. L. Falk, R. L. Somorjai, C. L. Lean, U. Himmelreich, J. Philips, P. Russell, B. Dolenko, A. E. Nikulin, and C. E. Mountford. Pathology of barretts esophagus by proton magnetic resonance spectroscopy and a statistical classification strategy. The American Journal of Surgery, 185(3):232-238, 2003.

[71] X. Yang and K. Murase. Tagged cardiac mr image segmentation by contrast enhancement and texture analysis. International Conference on Electronic Measurement Instruments,(ICEMI '09), pages 210-214, 2009.

[72] X. Liu and J. L. Prince. Shortest path refinement for motion estimation from tagged MR Images. IEEE Transactions on Medical Imaging, 29(8):1560-1572, 2010.

[73] L. Axel and L. Dougherty. Heart wall motion: Improved method of spatial modulation of magnetization for MR imaging. Radiology, 172(8):349-350, 1989.

[74] C. Sato, S. Naganawa, T. Nakamura, H. Kumada, S. Miura, O. Takizawa, and T. Ishigaki. Differentiation of noncancerous tissue and cancer lesions by apparent diffusion coefficient values in transition and peripheral zones of the prostate. Magnnetic Resonance Imaging, 21(10):258262, 2005.

[75] R. Bammer. Basic principles of diffusion-weighted imaging. European Journal Radiology, 45(43):169-184, 2003.

[76] P. C. Sundgren, Q. Dong, D. G'mez-Hassan, S. K. Mukherji, P. Maly, and R. Welsh. Diffusion tensor imaging of the brain: Review of clinical applications. Neuroradiology, 46(5), 2004. 
[77] D. L. Bihan, J. F. Mangin, C. Poupon, C. A. Clark, S. Pappata, N. Molko, and H. Chabriat. Diffusion tensor imaging: concepts and applications. Journal of Magnetic Resonance Imaging, 13(4):354-546, 2001.

[78] N. Barnea-Goraly, H. Kwon, V. Menon, S. Eliez, L. Lotspeich, and A. L. Reiss. White matter structure in autism: preliminary evidence from diffusion tensor imaging. Biological psychiatry, 55(3):323-326, 2004.

[79] W. B. Groen, J. K. Buitelaar, R. J. Van Der Gaag, and M. P. Zwiers. Pervasive microstructural abnormalities in autism: a dti study. Journal of psychiatry $\mathcal{E}$ neuroscience: JPN, 36(1):32, 2011.

[80] A. L. Alexander, J. E. Lee, M. Lazar, R. Boudos, M. B. DuBray, T. R. Oakes, J. N. Miller, J. Lu, E.-K. Jeong, W. M. McMahon, et al. Diffusion tensor imaging of the corpus callosum in autism. Neuroimage, 34(1):61-73, 2007.

[81] V. J. Wedeen, R. P. Wang, J. D. Schmahmann, T. Benner, W. Y. Tseng, G. Dai, D. N. Pandya, P. Hagmann, H. D'Arceuil, and A. J. de Crespigny. Diffusion spectrum magnetic resonance imaging (DSI) tractography of crossing fibers. Neuroimage, 41(4):1267-1277, 2008.

[82] P. Hagmann, L. Jonasson, P. Maeder, J.-P. Thiran, V. J. Wedeen, and R. Meuli. Understanding diffusion MR imaging techniques: From scalar diffusionweighted imaging to diffusion tensor imaging and beyond. Radiographics, 26(1):205-1203, 2006.

[83] S. Mori and J.-D. Tournier. Introduction to Diffusion Tensor Imaging 2e: And Higher Order Models. Academic Press, 2013.

[84] J. Crank. The mathematics of diffusion. Oxford university press, 1979.

[85] D. Zhou. Statistical analysis of diffusion tensor imaging. $\mathrm{PhD}$ thesis, University of Nottingham, 2010. 
[86] H. Johansen-Berg and T. E. Behrens. Diffusion MRI: From quantitative measurement to in-vivo neuroanatomy. Academic Press, 2009.

[87] E. Stejskal and J. Tanner. Spin diffusion measurements: Spin echoes in the presence of a time-dependent field gradient. The journal of chemical physics, 42(1):288-292, 2004.

[88] D. Le Bihan, E. Breton, D. Lallemand, P. Grenier, E. Cabanis, M. LavalJeantet, et al. Mr imaging of intravoxel incoherent motions: application to diffusion and perfusion in neurologic disorders. Radiology, 161(2):401-407, 1986.

[89] C.-F. Westin, S. E. Maier, H. Mamata, A. Nabavi, F. A. Jolesz, and R. Kikinis. Processing and visualization for diffusion tensor MRI. Medical image analysis, 6(2):93-108, 2002.

[90] N. Zhang, Z.-S. Deng, F. Wang, and X.-Y. Wang. The effect of different number of diffusion gradients on snr of diffusion tensor-derived measurement maps. Journal of Biomedical Science and Engineering, 2:96, 2009.

[91] C. G. Koay, L.-C. Chang, J. D. Carew, C. Pierpaoli, and P. J. Basser. A unifying theoretical and algorithmic framework for least squares methods of estimation in diffusion tensor imaging. Journal of Magnetic Resonance, 182(1):115$125,2006$.

[92] Y. Masutani, S. Aoki, O. Abe, N. Hayashi, and K. Otomo. Mr diffusion tensor imaging: recent advance and new techniques for diffusion tensor visualization. European journal of radiology, 46(1):53-66, 2003.

[93] C. L. Lawson and R. J. Hanson. Solving least squares problems, volume 161. SIAM, 1974. 
[94] A. Plaisier, K. Pieterman, M. Lequin, P. Govaert, A. Heemskerk, I. Reiss, G. Krestin, A. Leemans, and J. Dudink. Choice of diffusion tensor estimation approach affects fiber tractography of the fornix in preterm brain. American Journal of Neuroradiology, 2014.

[95] P. van Gelderen, M. H. de Vleeschouwer, D. DesPres, J. Pekar, P. van Zijl, and C. T. Moonen. Water diffusion and acute stroke. Magnetic Resonance in Medicine, 31(2):154-163, 1994.

[96] K. L. Narr, N. Hageman, R. P. Woods, L. S. Hamilton, K. Clark, O. Phillips, D. W. Shattuck, R. F. Asarnow, A. W. Toga, and K. H. Nuechterlein. Mean diffusivity: a biomarker for csf-related disease and genetic liability effects in schizophrenia. Psychiatry Research: Neuroimaging, 171(1):20-32, 2009.

[97] A. Lerner, M. A. Mogensen, P. E. Kim, M. S. Shiroishi, D. H. Hwang, and M. Law. Clinical applications of diffusion tensor imaging. World neurosurgery, 2013.

[98] D. Le Bihan, J.-F. Mangin, C. Poupon, C. A. Clark, S. Pappata, N. Molko, and H. Chabriat. Diffusion tensor imaging: concepts and applications. Journal of magnetic resonance imaging, 13(4):534-546, 2001.

[99] S.-K. Song, J. Yoshino, T. Q. Le, S.-J. Lin, S.-W. Sun, A. H. Cross, and R. C. Armstrong. Demyelination increases radial diffusivity in corpus callosum of mouse brain. Neuroimage, 26(1):132-140, 2005.

[100] P. G. Nucifora, R. Verma, S.-K. Lee, and E. R. Melhem. Diffusion-tensor MR imaging and tractography: exploring brain microstructure and connectivity. Radiology, 245(2):367, 2007.

[101] P. J. Basser, S. Pajevic, C. Pierpaoli, J. Duda, and A. Aldroubi. In vivo fiber tractography using dt-mri data. Magnetic resonance in medicine, 44(4):625-632, 2000. 
[102] A. Fedorov, R. Beichel, J. Kalpathy-Cramer, J. Finet, J.-C. Fillion-Robin, S. Pujol, C. Bauer, D. Jennings, F. Fennessy, M. Sonka, et al. 3D slicer as an image computing platform for the quantitative imaging network. Multidisciplinary Respiratory Medicine, 30(9):1323-1341, 2012.

[103] T. E. Conturo, N. F. Lori, T. S. Cull, E. Akbudak, A. Z. Snyder, J. S. Shimony, R. C. McKinstry, H. Burton, and M. E. Raichle. Tracking neuronal fiber pathways in the living human brain. Proceedings of the National Academy of Sciences, 96(18):10422-10427, 1999.

[104] P. J. Basser and D. K. Jones. Diffusion-tensor mri: theory, experimental design and data analysis-a technical review. NMR in Biomedicine, 15(7-8):456467, 2002.

[105] T. Behrens, M. Woolrich, M. Jenkinson, H. Johansen-Berg, R. Nunes, S. Clare, P. Matthews, J. Brady, and S. Smith. Characterization and propagation of uncertainty in diffusion-weighted $\mathrm{mr}$ imaging. Magnetic resonance in medicine, 50(5):1077-1088, 2003.

[106] A. Gropman, B. Gertz, K. Shattuck, I. Kahn, R. Seltzer, L. Krivitsky, and J. Van Meter. Diffusion tensor imaging detects areas of abnormal white matter microstructure in patients with partial ornithine transcarbamylase deficiency. American Journal of Neuroradiology, 31(9):1719-1723, 2010.

[107] P. H. Kamil Zelek, Cisrikov Viera. 2013.

[108] E. Rykhlevskaia, G. Gratton, and M. Fabiani. Combining structural and functional neuroimaging data for studying brain connectivity: a review. Psychophysiology, 45(2):173-187, 2008.

[109] T. Paus, A. Zijdenbos, K. Worsley, D. L. Collins, J. Blumenthal, J. N. Giedd, J. L. Rapoport, and A. C. Evans. Structural maturation of neural pathways in children and adolescents: in vivo study. Science, 283(5409):1908-1911, 1999. 
[110] P. McGraw, L. Liang, and J. M. Provenzale. Evaluation of normal age-related changes in anisotropy during infancy and childhood as shown by diffusion tensor imaging. American Journal of Roentgenology, 179(6):1515-1522, 2002.

[111] P. Mukherjee, J. H. Miller, J. S. Shimony, T. E. Conturo, B. C. Lee, C. R. Almli, and R. C. McKinstry. Normal brain maturation during childhood: Developmental trends characterized with diffusion-tensor mr imaging 1. Radiology, 221(2):349-358, 2001.

[112] D. M. N. S. Y. Developmental, . P. Investigators, et al. Prevalence of autism spectrum disorder among children aged 8 years-autism and developmental disabilities monitoring network, 11 sites, united states, 2010. Morbidity and mortality weekly report. Surveillance summaries (Washington, DC: 2002), 63:1, 2014.

[113] B. G. Travers, N. Adluru, C. Ennis, D. P. Tromp, D. Destiche, S. Doran, E. D. Bigler, N. Lange, J. E. Lainhart, and A. L. Alexander. Diffusion tensor imaging in autism spectrum disorder: a review. Autism Research, 5(5):289-313, 2012.

[114] M. F. Casanova, D. P. Buxhoeveden, and C. Brown. Clinical and macroscopic correlates of minicolumnar pathology in autism. Journal of Child Neurology, 17(9):692-695, 2002.

[115] M. F. Casanova, D. P. Buxhoeveden, A. E. Switala, and E. Roy. Neuronal density and architecture (gray level index) in the brains of autistic patients. Journal of Child Neurology, 17(7):515-521, 2002.

[116] M. F. Casanova, I. A. van Kooten, A. E. Switala, H. van Engeland, H. Heinsen, H. W. Steinbusch, P. R. Hof, J. Trippe, J. Stone, and C. Schmitz. Minicolumnar abnormalities in autism. Acta neuropathologica, 112(3):287-303, 2006. 
[117] S. E. Schipul, T. A. Keller, and M. A. Just. Inter-regional brain communication and its disturbance in autism. Front Syst Neurosci, 5(10):7, 2011.

[118] J. J. Wolff, H. Gu, G. Gerig, J. T. Elison, M. Styner, S. Gouttard, K. N. Botteron, S. R. Dager, G. Dawson, A. M. Estes, et al. Differences in white matter fiber tract development present from 6 to 24 months in infants with autism. American Journal of Psychiatry, 169(6):589-600, 2012.

[119] H. Arimura, T. Magome, Y. Yamashita, and D. Yamamoto. Computer-aided diagnosis systems for brain diseases in magnetic resonance images. Algorithms, 2(3):925-952, 2009.

[120] N. I. Weisenfeld and S. K. Warfield. Automatic segmentation of newborn brain MRI. Neuroimage, 47(2):564-572, 2009.

[121] T. P. Trouard, Y. Sabharwal, M. I. Altbach, and A. F. Gmitro. Analysis and comparison of motion-correction techniques in diffusion-weighted imaging. Journal of Magnetic Resonance Imaging, 6(6):925-935, 1996.

[122] H. Xue et al. Automatic segmentation and reconstruction of the cortex from neonatal MRI. Neuroimage, 38(3):461-477, 2007.

[123] A. U. Mewes, P. S. Hueppi, H. Als, F. J. Rybicki, T. E. Inder, G. B. McAnulty, R. V. Mulkern, R. L. Robertson, M. J. Rivkin, and S. K. Warfield. Regional brain development in serial magnetic resonance imaging of low-risk preterm infants. Pediatrics, 118(1):23-33, 2006.

[124] A. Barkovich. Magnetic resonance techniques in the assessment of myelin and myelination. Journal of inherited metabolic disease, 28(3):311-343, 2005.

[125] A. M. Mathur, J. J. Neil, R. C. McKinstry, and T. E. Inder. Transport, monitoring, and successful brain MR imaging in unsedated neonates. Pediatric radiology, 38(3):260-264, 2008. 
[126] P. Anbeek, K. L. Vincken, F. Groenendaal, A. Koeman, M. J. Van Osch, and J. Van der Grond. Probabilistic brain tissue segmentation in neonatal magnetic resonance imaging. Pediatric research, 63(2):158-163, 2008.

[127] M. J. Cardoso, A. Melbourne, G. S. Kendall, M. Modat, N. J. Robertson, N. Marlow, and S. Ourselin. Adapt: An adaptive preterm segmentation algorithm for neonatal brain mri. NeuroImage, 65:97-108, 2013.

[128] L. Gui, R. Lisowski, T. Faundez, P. S. Hüppi, F. Lazeyras, and M. Kocher. Morphology-driven automatic segmentation of MR images of the neonatal brain. Medical image analysis, 16(8):1565-1579, 2012.

[129] V. Grau, A. Mewes, M. Alcaniz, R. Kikinis, and S. K. Warfield. Improved watershed transform for medical image segmentation using prior information. Medical Imaging, IEEE Transactions on, 23(4):447-458, 2004.

[130] T. F. Chan and L. A. Vese. Active contours without edges. Image processing, IEEE transactions on, 10(2):266-277, 2001.

[131] L. Wang, F. Shi, G. Li, W. Lin, J. H. Gilmore, and D. Shen. Integration of sparse multi-modality representation and geometrical constraint for isointense infant brain segmentation. In Medical Image Computing and ComputerAssisted Intervention, (MICCAI'13), pages 703-710. Springer, 2013.

[132] M. Prastawa, J. H. Gilmore, W. Lin, and G. Gerig. Automatic segmentation of $\mathrm{mr}$ images of the developing newborn brain. Medical image analysis, 9(5):457466, 2005.

[133] S. K. Warfield, M. Kaus, F. A. Jolesz, and R. Kikinis. Adaptive, template moderated, spatially varying statistical classification. Medical image analysis, $4(1): 43-55,2000$. 
[134] F. Shi, Y. Fan, S. Tang, J. H. Gilmore, W. Lin, and D. Shen. Neonatal brain image segmentation in longitudinal mri studies. Neuroimage, 49(1):391-400, 2010.

[135] D. Shen and C. Davatzikos. Hammer: hierarchical attribute matching mechanism for elastic registration. Medical Imaging, IEEE Transactions on, 21(11):1421-1439, 2002.

[136] S. H. Kim, V. S. Fonov, C. Dietrich, C. Vachet, H. C. Hazlett, R. G. Smith, M. M. Graves, J. Piven, J. H. Gilmore, S. R. Dager, et al. Adaptive prior probability and spatial temporal intensity change estimation for segmentation of the one-year-old human brain. Journal of neuroscience methods, 212(1):43-55, 2013.

[137] H. C. Hazlett, M. D. Poe, G. Gerig, M. Styner, C. Chappell, R. G. Smith, C. Vachet, and J. Piven. Early brain overgrowth in autism associated with an increase in cortical surface area before age 2 years. Archives of general psychiatry, 68(5):467-476, 2011.

[138] V. Fonov, A. C. Evans, K. Botteron, C. R. Almli, R. C. McKinstry, and D. L. Collins. Unbiased average age-appropriate atlases for pediatric studies. Neuroimage, 54(1):313-327, 2011.

[139] J. T. Elison, S. J. Paterson, J. J. Wolff, J. S. Reznick, N. J. Sasson, H. Gu, K. N. Botteron, S. R. Dager, A. M. Estes, A. C. Evans, et al. White matter microstructure and atypical visual orienting in 7-month-olds at risk for autism. American Journal of Psychiatry, 2013.

[140] N. Sadeghi, M. Prastawa, P. T. Fletcher, J. Wolff, J. H. Gilmore, and G. Gerig. Regional characterization of longitudinal DT-MRI to study white matter maturation of the early developing brain. Neuroimage, 2012. 
[141] F. Leroy, J.-F. Mangin, F. Rousseau, H. Glasel, L. Hertz-Pannier, J. Dubois, and G. Dehaene-Lambertz. Atlas-free surface reconstruction of the cortical grey-white interface in infants. PloS one, 6(11):e27128, 2011.

[142] R. M. Haralick, S. R. Sternberg, and X. Zhuang. Image analysis using mathematical morphology. Pattern Analysis and Machine Intelligence, IEEE Transactions on, (4):532-550, 1987.

[143] J.-P. Thirion and A. Gourdon. Computing the differential characteristics of isointensity surfaces. Computer vision and image understanding, 61(2):190-202, 1995.

[144] J.-F. Mangin, D. Riviere, A. Cachia, E. Duchesnay, Y. Cointepas, D. Papadopoulos-Orfanos, P. Scifo, T. Ochiai, F. Brunelle, and J. Regis. A framework to study the cortical folding patterns. Neuroimage, 23:S129-S138, 2004.

[145] L. Wang, F. Shi, W. Lin, J. H. Gilmore, and D. Shen. Automatic segmentation of neonatal images using convex optimization and coupled level sets. NeuroImage, 58(3):805-817, 2011.

[146] L. Wang, L. He, A. Mishra, and C. Li. Active contours driven by local gaussian distribution fitting energy. Signal Processing, 89(12):2435-2447, 2009.

[147] L. Wang, F. Shi, P.-T. Yap, W. Lin, J. H. Gilmore, and D. Shen. Longitudinally guided level sets for consistent tissue segmentation of neonates. Human brain mapping, 2011.

[148] D. L. Pham and J. L. Prince. Adaptive fuzzy segmentation of magnetic resonance images. Medical Imaging, IEEE Transactions on, 18(9):737-752, 1999.

[149] L. Wang, F. Shi, P.-T. Yap, J. H. Gilmore, W. Lin, and D. Shen. 4D multi- 
modality tissue segmentation of serial infant images. PloS one, 7(9):e44596, 2012.

[150] L. Wang, F. Shi, G. Li, Y. Gao, W. Lin, J. H. Gilmore, and D. Shen. Segmentation of neonatal brain $\mathrm{mr}$ images using patch-driven level sets. NeuroImage, 84:141-158, 2014.

[151] P. Savadjiev, Y. Rathi, S. Bouix, A. R. Smith, R. T. Schultz, R. Verma, and C.-F. Westin. Combining surface and fiber geometry: An integrated approach to brain morphology. In Proceedings of Medical Image Computing and ComputerAssisted Intervention (MICCAI'13), pages 50-57. 2013.

[152] Z. Liu, Y. Wang, G. Gerig, S. Gouttard, R. Tao, T. Fletcher, and M. Styner. Quality control of diffusion weighted images. In Proceedings of SPIE Medical Imaging 2000: Image Processing (SPIE'10), pages 76280J-76280J, 2010.

[153] A. Tristán-Vega and S. Aja-Fernández. Dwi filtering using joint information for dti and hardi. Medical Image Analysis, 14(2):205-218, 2010.

[154] A. J. van der Kouwe, T. Benner, D. H. Salat, and B. Fischl. Brain morphometry with multiecho mprage. Neuroimage, 40(2):559-569, 2008.

[155] S. M. Smith. Fast robust automated brain extraction. Human brain mapping, 17(3):143-155, 2002.

[156] J.-X. Liu, Y.-S. Chen, and L.-F. Chen. Accurate and robust extraction of brain regions using a deformable model based on radial basis functions. Journal of neuroscience methods, 183(2):255-266, 2009.

[157] A. H. Zhuang, D. J. Valentino, and A. W. Toga. Skull-stripping magnetic resonance brain images using a model-based level set. NeuroImage, 32(1):79_ 92, 2006. 
[158] J. Ashburner and K. J. Friston. Voxel-based morphometrythe methods. Neuroimage, 11(6):805-821, 2000.

[159] K. K. Leung, J. Barnes, G. R. Ridgway, J. W. Bartlett, M. J. Clarkson, K. Macdonald, N. Schuff, N. C. Fox, and S. Ourselin. Automated cross-sectional and longitudinal hippocampal volume measurement in mild cognitive impairment and alzheimer's disease. Neuroimage, 51(4):1345-1359, 2010.

[160] J. E. Iglesias, C.-Y. Liu, P. M. Thompson, and Z. Tu. Robust brain extraction across datasets and comparison with publicly available methods. IEEE Transactions on Medical Imaging, 30(9):1617-1634, 2011.

[161] F. Segonne, A. Dale, E. Busa, M. Glessner, D. Salat, H. Hahn, and B. Fischl. A hybrid approach to the skull stripping problem in mri. Neuroimage, 22(3):1060-1075, 2004.

[162] D. E. Rex, D. W. Shattuck, R. P. Woods, K. L. Narr, E. Luders, K. Rehm, S. E. Stolzner, D. A. Rottenberg, and A. W. Toga. A meta-algorithm for brain extraction in mri. NeuroImage, 23(2):625-637, 2004.

[163] A. Farag, A. El-Baz, and G. Gimel'farb. Precise segmentation of multimodal images. IEEE Transaction on Image Processssing, 15(4):952-968, 2006.

[164] A. El-Baz, A. Elnakib, F. Khalifa, M. A. El-Ghar, P. McClure, A. Soliman, and G. Gimel'farb. Precise segmentation of 3-D magnetic resonance angiography. IEEE Transaction on Biomedical Engineering, 59(7):2019-2029, 2012.

[165] C. Bouman and K. Sauer. A generalized gaussian image model for edgepreserving map estimation. IEEE Transactions on Image Processing, 2(3):296310, 1993.

[166] S. Boyd and L. Vandenberghe. Convex optimization. Cambridge university press, 2009. 
[167] D. D. Lee and H. S. Seung. Learning the parts of objects by non-negative matrix factorization. Nature, 401(6755):788-791, 1999.

[168] Y. Xie, J. Ho, and B. C. Vemuri. Nonnegative factorization of diffusion tensor images and its applications. In Inform. Process. Med. Imaging, pages 550-561. Springer, 2011.

[169] T. Pang-Ning, M. Steinbach, V. Kumar, et al. Introduction to data mining. WP Co, 2006.

[170] M. W. Berry, M. Browne, A. N. Langville, V. P. Pauca, and R. J. Plemmons. Algorithms and applications for approximate nonnegative matrix factorization. Computational Statistics \& Data Analysis, 52(1):155-173, 2007.

[171] A. Albert. Regression and the Moore-Penrose pseudoinverse. Elsevier, 1972.

[172] R. O. Duda, P. E. Hart, and D. G. Stork. Pattern classification. John Wiley \& Sons, 2012.

[173] P. A. Viola and W. M. W. III. Alignment by maximization of mutual information. International Journal on Computer Vision, 24(2):137-154, 1997.

[174] F. Shi, P.-T. Yap, G. Wu, H. Jia, J. H. Gilmore, W. Lin, and D. Shen. Infant brain atlases from neonates to 1-and 2-year-olds. PLoS One, 6(4):e18746, 2011.

[175] J. Ashburner and K. J. Friston. Unified segmentation. Neuroimage, 26(3):839851, 2005.

[176] Statistical Parametric Mapping (SPM). http://www.fil.ion.ucl.ac . $\mathrm{uk} / \mathrm{spm} /$.

[177] A. El-Baz. Novel stochastic models for medical image analysis. PhD thesis, University of Louisville, Louisville, KY, USA, 2006. 
[178] J. Besag. Spatial interaction and the statistical analysis of lattice systems. Journal of the Royal Statistical Society. Series B (Methodological), pages 192-236, 1974.

[179] K. O. Babalola, B. Patenaude, P. Aljabar, J. Schnabel, D. Kennedy, W. Crum, S. Smith, T. Cootes, M. Jenkinson, and D. Rueckert. An evaluation of four automatic methods of segmenting the subcortical structures in the brain. Neuroimage, 47(4):1435-1447, 2009.

[180] Infant Brain Imaging Study (IBIS). http://www. ibisnetwork . org/.

[181] M. L. Ganz. The lifetime distribution of the incremental societal costs of autism. Archives of Pediatrics \& Adolescent Medicine, 161(4):343-349, 2007.

[182] G. Gerig, M. Styner, D. Jones, D. Weinberger, and J. Lieberman. Shape analysis of brain ventricles using spharm. In Mathematical Methods in Biomedical Image Analysis, 2001. MMBIA 2001. IEEE Workshop on, pages 171-178. IEEE, 2001.

[183] M. K. Chung, K. M. Dalton, L. Shen, A. C. Evans, and R. J. Davidson. Weighted fourier series representation and its application to quantifying the amount of gray matter. Medical Imaging, IEEE Transactions on, 26(4):566-581, 2007.

[184] Q. Fang and D. A. Boas. Tetrahedral mesh generation from volumetric binary and grayscale images. In Proceedings of IEEE International Symposium on Biomedical Imaging: From Nano to Macro (ISBI'09), pages 1142-1145. IEEE, 2009.

[185] M. Nitzken, M. Casanova, G. Gimel'farb, F. Khalifa, A. Elnakib, A. E. Switala, and A. El-Baz. 3D shape analysis of the brain cortex with application to autism. In Proceedings of IEEE International Symposium on Biomedical Imaging: From Nano to Macro (ISBI'11), pages 1847-1850. IEEE, 2011. 
[186] R. Courant and D. Hilbert. Methods of Mathematical Physics, volume 1. London: Interscience, 1953.

[187] L. Shen and M. K. Chung. Large-scale modeling of parametric surfaces using spherical harmonics. In Third International Symposium on 3D Data Processing, Visualization, and Transmission, pages 294-301. IEEE, 2006.

[188] F. Khalifa, G. Beache, M. Abou El-Ghar, T. El-Diasty, G. Gimelfarb, M. Kong, and A. El-Baz. Dynamic contrast-enhanced mri-based early detection of acute renal transplant rejection. 2013.

[189] M. Mostapha, A. Alansary, A. Soliman, F. Khalifa, M. Nitzken, R. Khodeir, M. F. Casanova, and A. El-Baz. Atlas-based approach for the segmentation of infant dti mr brain images. In Proceedings of IEEE International Symposium on Biomedical Imaging: From Nano to Macro (ISBI'14), pages 1255-1258, 2014.

[190] M. Mostapha, A. Soliman, F. Khalifa, A. Elnakib, A. Alansary, M. Nitzken, M. F. Casanova, and A. El-Baz. A Statistical Framework for the Classification of Infant DT Images. In Proceedings IEEE International Conference on Image Processing (ICIP'14), page in press, Paris, France, October 27-30, 2014.

[191] A. El-Baz, M. F. Casanova, G. Gimel'farb, M. Mott, and A. E. Switala. Autism diagnostics by 3D texture analysis of cerebral white matter gyrifications. In Proc. International Conference on Medical Image Computing and ComputerAssisted Intervention (MICCAI'2007), pages 882-890. Springer, 2007.

[192] M. F. Casanova, A. El-Baz, M. Mott, G. Mannheim, H. Hassan, R. Fahmi, J. Giedd, J. M. Rumsey, A. E. Switala, and A. Farag. Reduced gyral window and corpus callosum size in autism: Possible macroscopic correlates of a minicolumnopathy. Journal of Autism and Developmental Disorders, 39(5):751764, 2009. 
[193] M. F. Casanova, A. Farag, E.-B. Ayman, M. Meghan, H. Hassan, R. Fahmi, and A. E. Switala. Abnormalities of the gyral window in autism: A macroscopic correlate to a putative minicolumnopathy. Journal of Special Education and Rehabilitation, 7(1-2), 2006.

[194] R. Fahmi, A. S. El-Baz, H. Abd El Munim, A. A. Farag, and M. F. Casanova. Classification techniques for autistic vs. typically developing brain using MRI data. In Proc. IEEE International Symposium on Biomedical Imaging: From Nano to Macro (ISBI'2007), pages 1348-1351. IEEE, 2007.

[195] A. A. Farag, R. Fahmi, M. F. Casanova, A. E. Abdel-Hakim, H. A. El-Munim, and A. El-Baz. Robust neuroimaging-based classification techniques of autistic vs. typically developing brain. In Deformable Models, chapter 16, pages 535-566. Springer, 2007.

[196] R. Fahmi, A. Elbaz, H. Hassan, A. A. Farag, and M. F. Casanova. Structural MRI-based discrimination between autistic and typically developing brain. In Proc. Computer Assisted Radiology and Surgery (CARS'2007), pages 24-26, 2007.

[197] M. F. Casanova, A. El-Baz, E. Vanbogaert, P. Narahari, and A. Switala. A topographic study of minicolumnar core width by lamina comparison between autistic subjects and controls: Possible minicolumnar disruption due to an anatomical element in-common to multiple laminae. Brain Pathology, 20(2):451-458, 2010.

[198] M. F. Casanova, A. S. El-Baz, S. S. Kamat, B. A. Dombroski, F. Khalifa, A. Elnakib, A. Soliman, A. Allison-McNutt, and A. E. Switala. Focal cortical dysplasias in autism spectrum disorders. Acta Neuropathologica Communications, 1(1):67, 2013. 
[199] M. Nitzken, M. Casanova, G. Gimel'farb, A. Elnakib, F. Khalifa, A. Switala, and A. El-Baz. 3d shape analysis of the brain cortex with application to dyslexia. In Image Processing (ICIP), 2011 18th IEEE International Conference on, pages 2657-2660. IEEE, 2011.

[200] E. L. Williams, A. El-Baz, M. Nitzken, A. E. Switala, and M. F. Casanova. Spherical harmonic analysis of cortical complexity in autism and dyslexia. Translational neuroscience, 3(1):36-40, 2012.

[201] Radiologyinfo.org. The radiology information resource for patients. http: //www.radiologyinfo.org/en/info.cfm?pg=fmribrain.

[202] S. E. Yuksel, A. El-Baz, A. A. Farag, M. Abo El-Ghar, T. A. Eldiasty, and M. A. Ghoneim. Automatic detection of renal rejection after kidney transplantation. In International Congress Series, volume 1281, pages 773-778, 2005.

[203] S. E. Yuksel, A. El-Baz, and A. A. Farag. A kidney segmentation framework for dynamic contrast enhanced magnetic resonance imaging. In Proc. International Symposium on Mathematical Methods in Engineering (MME'2006), pages 55-64, Ankara, Turkey, April, 27-29, 2006.

[204] S. E. Yuksel, A. El-Baz, A. A. Farag, M. El-Ghar, T. Eldiasty, and M. A. Ghoneim. A kidney segmentation framework for dynamic contrast enhanced magnetic resonance imaging. Journal of Vibration and Control, 13(910):1505-1516, 2007.

[205] A. M. Ali, A. A. Farag, and A. El-Baz. Graph cuts framework for kidney segmentation with prior shape constraints. In Proc. International Conference on Medical Image Computing and Computer-Assisted Intervention (MICCAI'2007), volume 1, pages 384-392, Brisbane, Australia, October 29-November 2, 2007.

[206] A. El-Baz, A. Farag, R. Fahmi, S. Yuksel, W. Miller, M. A. El-Ghar, T. ElDiasty, and M. Ghoneim. A new cad system for the evaluation of kidney 
diseases using dce-mri. In Proceedings International Conference Medical Image Computing and Computer-Assisted Intervention, MICCAI-2006, pages 446-453, Copenhagen, Denmark, October 1-6, 2006.

[207] F. Khalifa, G. Gimel'farb, M. A. El-Ghar, G. Sokhadze, S. Manning, P. McClure, R. Ouseph, and A. El-Baz. A new deformable model-based segmentation approach for accurate extraction of the kidney from abdominal CT images. In Proc. International Conference on Image Processing (ICIP'2011), pages 3393-3396, Brussels, Belgium, September 11-14, 2011.

[208] F. Khalifa, A. Elnakib, G. M. Beache, G. Gimel'farb, M. A. El-Ghar, G. Sokhadze, S. Manning, P. McClure, and A. El-Baz. 3D kidney segmentation from CT images using a level set approach guided by a novel stochastic speed function. In Proc. International Conference on Medical Image Computing and Computer-Assisted Intervention (MICCAI'2011), pages 587-594, Toronto, Canada, Sept. 18-22, 2011.

[209] F. Khalifa, M. A. El-Ghar, B. Abdollahi, H. Frieboes, T. El-Diasty, and A. ElBaz. A comprehensive non-invasive framework for automated evaluation of acute renal transplant rejection using DCE-MRI. NMR in Biomedicine, 26(11):1460-1470, 2013.

[210] M. Mostapha, F. Khalifa, A. Alansary, A. Soliman, G. Gimel'farb, and A. ElBaz. Dynamic MRI-based computer aided diagnostic systems for early detection of kidney transplant rejection: A survey. In Proc. International Symposium on Computational Models for Life Sciences (CMLS'2013), volume 1559, pages 297-306, 2013.

[211] M. Mostapha, F. Khalifa, A. Alansary, A. Soliman, J. Suri, and A. ElBaz. Computer-aided diagnosis systems for acute renal transplant rejection: 
Challenges and methodologies. In A. El-Baz and L. saba J. Suri, editors, $A b$ domen and Thoracic Imaging, chapter 1, pages 1-35. Springer, 2014.

[212] A. El-Baz, A. A. Farag, R. Falk, and R. La Rocca. Automatic identification of lung abnormalities in chest spiral CT scans. In Proc. IEEE International Conference on Acoustics, Speech, and Signal Processing, (ICASSP'2003), volume 2, pages II-261. IEEE, 2003.

[213] A. El-Baz, A. A. Farag, R. Falk, and R. La Rocca. A unified approach for detection, visualization, and identification of lung abnormalities in chest spiral CT scans. In International Congress Series, volume 1256, pages 998-1004. Elsevier, 2003.

[214] A. A. Farag, A. El-Baz, G. Gimel'farb, and R. Falk. Detection and recognition of lung abnormalities using deformable templates. In Proc. International Conference on Pattern Recognition (ICPR'2004), volume 3, pages 738-741. IEEE, 2004.

[215] A. A. Farag, A. El-Baz, G. G. Gimelfarb, R. Falk, and S. G. Hushek. Automatic detection and recognition of lung abnormalities in helical CT images using deformable templates. In Proc. International Conference on Medical Image Computing and Computer-Assisted Intervention (MICCAI'2004), pages 856-864. Springer, 2004.

[216] A. Farag, A. El-Baz, G. Gimel'farb, and R. Falk. Detection and recognition of lung nodules in spiral CT images using deformable templates and Bayesian post-classification. In Proc. International Conference on Image Processing (ICIP'2004), volume 5, pages 2921-2924. IEEE, 2004.

[217] A. El-Baz, S. E. Yuksel, S. Elshazly, and A. A. Farag. Non-rigid registration techniques for automatic follow-up of lung nodules. In Proc. Computer 
Assisted Radiology and Surgery (CARS'2005), volume 1281, pages 1115-1120. Elsevier, 2005.

[218] A. A. Farag, A. El-Baz, G. Gimelfarb, M. Abou El-Ghar, and T. Eldiasty. Quantitative nodule detection in low dose chest CT scans: New template modeling and evaluation for CAD system design. In Proc. International Conference on Medical Image Computing and Computer-Assisted Intervention (MICCAI'2005), pages 720-728. Springer, 2005.

[219] A. El-Baz, A. Farag, G. Gimel'farb, R. Falk, M. Abou El-Ghar, and T. Eldiasty. A framework for automatic segmentation of lung nodules from low dose chest CT scans. In Proc. International Conference on Pattern Recognition (ICPR'2006), volume 3, pages 611-614. IEEE, 2006.

[220] A. M. Ali, A. S. El-Baz, and A. A. Farag. A novel framework for accurate lung segmentation using graph cuts. In Proc. IEEE International Symposium on Biomedical Imaging: From Nano to Macro (ISBI'2007), pages 908-911. IEEE, 2007.

[221] A. El-Baz, G. Gimel'farb, R. Falk, and M. Abou El-Ghar. A novel approach for automatic follow-up of detected lung nodules. In Proc. International Conference on Image Processing (ICIP'2007), volume 5, pages 501-504. IEEE, 2007.

[222] A. El-Baz, M. F. Casanova, G. Gimel'farb, M. Mott, and A. E. Switwala. A new image analysis approach for automatic classification of autistic brains. In Proc. IEEE International Symposium on Biomedical Imaging: From Nano to Macro (ISBI'2007), pages 352-355. IEEE, 2007.

[223] A. A. Farag, A. El-Baz, G. Gimel'farb, R. Falk, M. A. El-Ghar, T. Eldiasty, and S. Elshazly. Appearance models for robust segmentation of pulmonary nodules in 3D LDCT chest images. In Proc. International Conference on Medi- 
cal Image Computing and Computer-Assisted Intervention (MICCAI'2006), pages 734-741, Copenhagen, Denmark, October 1-6, 2006.

[224] A. M. Ali and A. A. Farag. Automatic lung segmentation of volumetric lowdose CT scans using graph cuts. In Advances in Visual Computing, pages 258267. Springer, 2008.

[225] A. El-Baz, G. L. Gimel'farb, R. Falk, M. Abou El-Ghar, T. Holland, and T. Shaffer. A new stochastic framework for accurate lung segmentation. In Proc. International Conference on Medical Image Computing and ComputerAssisted Intervention (MICCAI'2008), pages 322-330, 2008.

[226] A. El-Baz, G. L. Gimel'farb, R. Falk, D. Heredis, and M. Abou El-Ghar. A novel approach for accurate estimation of the growth rate of the detected lung nodules. In Proc. International Workshop on Pulmonary Image Analysis, pages 33-42, 2008.

[227] A. El-Baz, G. L. Gimel'farb, R. Falk, T. Holland, and T. Shaffer. A framework for unsupervised segmentation of lung tissues from low dose computed tomography images. In Proc. British Machine Vision (BMVC'2008), pages 1-10, 2008.

[228] A. El-Baz, G. Gimel'farb, R. Falk, and M. Abou El-Ghar. A new approach for automatic analysis of 3D low dose CT images for accurate monitoring the detected lung nodules. In Proc. International Conference on Pattern Recognition (ICPR'2008), pages 1-4. IEEE, 2008.

[229] A. El-Baz, G. Gimel'farb, R. Falk, M. Abou El-Ghar, and H. Refaie. Promising results for early diagnosis of lung cancer. In Proc. IEEE International Symposium on Biomedical Imaging: From Nano to Macro (ISBI'2008), pages 1151-1154. IEEE, 2008. 
[230] A. El-Baz, G. Gimelfarb, R. Falk, and M. Abo El-Ghar. Automatic analysis of 3D low dose CT images for early diagnosis of lung cancer. Pattern Recognition, 42(6):1041-1051, 2009.

[231] A. El-Baz, G. Gimelfarb, R. Falk, M. Abou El-Ghar, S. Rainey, D. Heredia, and T. Shaffer. Toward early diagnosis of lung cancer. In Proc. International Conference on Medical Image Computing and Computer-Assisted Intervention (MICCAI'2009), pages 682-689. Springer, 2009.

[232] A. El-Baz, G. Gimel'farb, R. Falk, and M. El-Ghar. Appearance analysis for diagnosing malignant lung nodules. In Proc. IEEE International Symposium on Biomedical Imaging: From Nano to Macro (ISBI'2010), pages 193-196. IEEE, 2010.

[233] A. El-Baz, P. Sethu, G. Gimel'farb, F. Khalifa, A. Elnakib, R. Falk, and M. Abo El-Ghar. Elastic phantoms generated by microfluidics technology: Validation of an imaged-based approach for accurate measurement of the growth rate of lung nodules. Biotechnology Journal, 6(2):195-203, 2011.

[234] A. El-Baz, G. Gimelfarb, R. Falk, M. Abo El-Ghar, and J. Suri. Appearance analysis for the early assessment of detected lung nodules. In Lung Imaging and Computer Aided Diagnosis, chapter 17, pages 395-404. CRC Press, 2011.

[235] A. El-Baz, P. Sethu, G. Gimelfarb, F. Khalifa, A. Elnakib, R. Falk, A. M. ElGhar, and J. Suri. Validation of a new imaged-based approach for the accurate estimating of the growth rate of detected lung nodules using real computed tomography images and elastic phantoms generated by state-oftheart microfluidics technology. In Lung Imaging and Computer Aided Diagnosis, chapter 18, pages 405-420. CRC Press, 2011.

[236] A. El-Baz, M. Nitzken, G. Gimelfarb, E. Van Bogaert, R. Falk, M. Abo El-Ghar, and J. Suri. Three-dimensional shape analysis using spherical harmonics for 
early assessment of detected lung nodules. In Lung Imaging and Computer Aided Diagnosis, chapter 19, pages 421-438. CRC Press, 2011.

[237] B. Abdollahi, A. Soliman, A. Civelek, X.-F. Li, G. Gimel'farb, and A. ElBaz. A novel gaussian scale space-based joint MGRF framework for precise lung segmentation. In Proc. International Conference on Image Processing (ICIP'2012), pages 2029-2032. IEEE, 2012.

[238] A. El-Baz, F. Khalifa, A. Elnakib, M. Nitzken, A. Soliman, P. McClure, M. Abou El-Ghar, and G. Gimelfarb. A novel approach for global lung registration using 3D Markov-Gibbs appearance model. In Proc. International Conference on Medical Image Computing and Computer-Assisted Intervention (MICCAI'2012), pages 114-121. Springer, 2012.

[239] A. El-Baz, G. Gimel'farb, M. Abou El-Ghar, and R. Falk. Appearance-based diagnostic system for early assessment of malignant lung nodules. In Proc. International Conference on Image Processing (ICIP'2012), pages 533-536. IEEE, 2012.

[240] A. El-Baz, A. Soliman, P. McClure, G. Gimel'farb, M. Abou El-Ghar, and R. Falk. Early assessment of malignant lung nodules based on the spatial analysis of detected lung nodules. In Proc. IEEE International Symposium on Biomedical Imaging: From Nano to Macro (ISBI'2012), pages 1463-1466. IEEE, 2012.

[241] A. Soliman, F. Khalifa, A. Alansary, G. Gimel'farb, and A. El-Baz. Segmentation of lung region based on using parallel implementation of joint MGRF: Validation on 3D realistic lung phantoms. In Proc. IEEE International Symposium on Biomedical Imaging: From Nano to Macro (ISBI'2013), pages 852-855. IEEE, 2013. 
[242] A. El-Baz, A. Elnakib, M. Abou El-Ghar, G. Gimel'farb, R. Falk, and A. Farag. Automatic detection of 2D and 3D lung nodules in chest spiral CT scans. International Journal of Biomedical Imaging, 2013, 2013.

[243] A. El-Baz, G. M. Beache, G. Gimel'farb, K. Suzuki, K. Okada, A. Elnakib, A. Soliman, and B. Abdollahi. Computer-aided diagnosis systems for lung cancer: Challenges and methodologies. International Journal of Biomedical Imaging, 2013, 2013.

[244] A. El-Baz, M. Nitzken, F. Khalifa, A. Elnakib, G. Gimelfarb, R. Falk, and M. Abo El-Ghar. 3D shape analysis for early diagnosis of malignant lung nodules. In Proc. Information Processing in Medical Imaging, (IPMI'2011), pages 772-783. Springer, 2011.

[245] A. Soliman, F. Khalifa, A. Alansary, G. Gimel'farb, and A. El-Baz. Performance evaluation of an automatic MGRF-based lung segmentation approach. In Proc. International Symposium on Computational Models for Life Sciences (CMLS'2013), volume 1559, pages 323-332, 2013.

[246] A. Firjany, A. Elnakib, A. El-Baz, G. Gimel'farb, M. A. El-Ghar, and A. Elmagharby. Novel stochastic framework for accurate segmentation of prostate in dynamic contrast enhanced MRI. In Prostate Cancer Imaging. Computer-Aided Diagnosis, Prognosis, and Intervention, pages 121-130. Springer, 2010.

[247] A. Firjani, A. Elnakib, F. Khalifa, A. El-Baz, G. Gimel'farb, M. A. El-Ghar, and A. Elmaghraby. A novel 3D segmentation approach for segmenting the prostate from dynamic contrast enhanced MRI using current appearance and learned shape prior. In Proc. IEEE International Symposium on Signal Processing and Information Technology (ISSPIT'2010), pages 137-143. IEEE, 2010. 
[248] A. Firjani, A. Elnakib, F. Khalifa, G. Gimel'farb, M. A. El-Ghar, J. Suri, A. Elmaghraby, and A. El-Baz. A new 3D automatic segmentation framework for accurate segmentation of prostate from DCE-MRI. In Proc. IEEE International Symposium on Biomedical Imaging: From Nano to Macro (ISIB'2011), pages 1476-1479. IEEE, 2011.

[249] A. Firjani, F. Khalifa, A. Elnakib, G. Gimel'farb, M. A. El-Ghar, A. Elmaghraby, and A. El-Baz. 3D automatic approach for precise segmentation of the prostate from diffusion-weighted magnetic resonance imaging. In Proc. IEEE International Conference on Image Processing (ICIP'2011), pages 2285-2288. IEEE, 2011.

[250] A. Firjani, A. Elnakib, F. Khalifa, G. Gimel'farb, M. A. El-Ghar, A. Elmaghraby, and A. El-Baz. A new 3D automatic segmentation framework for accurate extraction of prostate from diffusion imaging. In Proc. Biomedical Sciences and Engineering Conference (BSEC'2011), pages 1-4. IEEE, 2011.

[251] A. Firjani, A. Elnakib, F. Khalifa, G. Gimel'farb, M. A. El-Ghar, A. Elmaghraby, and A. El-Baz. A diffusion-weighted imaging based diagnostic system for early detection of prostate cancer. Journal of Biomedical Science and Engineering (JBiSE), 6:346-356, 2013.

[252] A. Firjani, F. Khalifa, A. Elnakib, G. Gimel'farb, M. A. El-Ghar, A. Elmaghraby, and A. El-Baz. A novel image-based approach for early detection of prostate cancer using DCE-MRI. In K. Suzuki, editor, Computational Intelligence in Biomedical Imaging, chapter 3, pages 55-85. Springer Science and Business Media, 2014.

[253] R. C. Dubes and A. K. Jain. Random field models in image analysis. Journal of Applied Statistics, 16(2):131-164, 1989. 
[254] G. L. Gimel'farb and A. Zalesny. Probabilistic models of digital region maps based on Markov random fields with short-and long-range interaction. Pattern Recognition Letters, 14(10):789-797, 1993.

[255] R. W. Picard and I. M. Elfadel. Structure of aura and co-occurrence matrices for the Gibbs texture model. Journal of Mathematical Imaging and Vision, 2(1):5-25, 1992.

[256] I. L. Dryden, M. R. Scarr, and C. C. Taylor. Bayesian texture segmentation of weed and crop images using reversible jump Markov chain Monte Carlo methods. Journal of the Royal Statistical Society: Series C (Applied Statistics), 52(1):31-50, 2003.

[257] G. L. Gimel'farb. Image textures and Gibbs random fields, volume 16. Springer, 1999. 


\section{APPENDIX A}

\section{Analytical Estimation of the bi-valued Gibbs Potentials}

Let $\mathbf{Q}=\{0, \ldots, Q-1\}$ and $\mathbf{L}=\{0, \ldots, L-1\}$ denote sets of gray levels $q$ and region labels $k$, respectively. Here, $Q$ is the number of gray levels and $K$ is the number of image modes, i.e. peaks in the gray level frequency distribution, e.g., for a bimodal image, $L=2$. Each dominant image mode is assumed to correspond to a particular class of objects to be found in the image.

Let $\mathbf{R}=\{(x, y, z): 0 \leq x \leq X-1,0 \leq y \leq Y-1,0 \leq z \leq Z-1\}$ be a 3D $(x, y, z)$-arithmetic grid supporting gray level images $\mathbf{g}: \mathbf{R} \rightarrow \mathbf{Q}$ and their region maps $\mathbf{m}: \mathbf{R} \rightarrow \mathbf{L}$. A two-level probability model of original images to segment and their desired region maps is given by a joint distribution $P(\mathbf{g}, \mathbf{m})=P(\mathbf{m}) P(\mathbf{g} \mid \mathbf{m})$ where $P(\mathbf{m})$ is an unconditional probability distribution of maps (second-order spatial Markov Gibbs random field (MGRF) model) and $P(\mathbf{g} \mid \mathbf{m})$ is a conditional distribution of images, given the map (first-order intensity model). The Bayesian maximum a posteriori (MAP) estimate of the map $\mathbf{m}$, given the image $\mathbf{g}$ :

$$
\mathbf{m}^{*}=\arg \max _{\mathbf{m} \in \mathcal{X}} L(\mathbf{g}, \mathbf{m})
$$

where $\mathcal{X}$ is the set of all region maps with labels $\lambda \in \mathbf{L}$ on $\mathbf{R}$, maximizes the loglikelihood function:

$$
L(\mathbf{g}, \mathbf{m})=\frac{1}{|\mathbf{R}|}(\log P(\mathbf{g} \mid \mathbf{m})+\log P(\mathbf{m}))
$$

To find this estimate, the first-order intensity model and second-order spatial MGRF model are selected, and their parameters are identified as well. 


\section{Unconditional Region Map Model}

The simplest model of interdependent region labels is the MGRF with the nearest 26-neighborhood of each voxel. By symmetry considerations, we assume the Gibbs potentials are independent of relative orientation of voxel pairs, are the same for all classes, and depend only on whether the pair of labels are equal or not. Under these assumptions, it is the simplest auto-binomial model, the Potts one, being for a long time a popular region map model [253-256]. However unlike the conventional counterparts, its Gibbs potential is obtained analytically using the maximum likelihood estimator for a generic MGRF derived in [257]. The 26neighborhood results in a family $\mathbf{C}_{\mathbf{N}}=\left[\mathbf{c}_{x, y, z, \xi, \eta, \kappa}=((x, y, z),(x+\xi, y+\eta, z+\kappa))\right.$ : $\left.(x, y, z) \in \mathbf{R} ;(x+\xi, y+\eta, z+\kappa) \in \mathbf{R} ;(\xi, \eta, \kappa) \in \nu_{s}\right]$ of the neighboring voxel pairs supporting the Gibbs potentials. The potentials are bi-valued because only the coincidence of the labels is taken into account: $V\left(\lambda, \lambda^{\prime}\right)=V_{\text {eq }}$ if $\lambda^{\prime}=\lambda^{\prime}$ and $V_{\text {ne }}$ if $\lambda \neq \lambda^{\prime}$. Then the MGRF model of region maps is as follows:

$$
\begin{aligned}
P(\mathbf{m}) & =\frac{1}{Z_{\mathbf{N}}} \exp \sum_{(x, y, z) \in \mathbf{R}} \sum_{(\xi, \eta, \kappa) \in \nu_{s}} V\left(m_{x, y, z}, m_{x+\xi, y+\eta, z+\kappa}\right) \\
& =\frac{1}{Z_{\mathbf{N}}} \exp \left(\left|\mathbf{C}_{\mathbf{N}}\right| V_{\text {eq }}\left(2 f_{\text {eq }}(\mathbf{m})-1\right)\right)
\end{aligned}
$$

where $\left|\mathbf{C}_{\mathbf{N}}\right|$ is the cardinality of the family $\mathbf{C}_{\mathbf{N}}$ and $f_{\text {eq }}(\mathbf{m})$ denotes the relative frequency of the equal labels in the voxel pairs of this family:

$$
f_{\text {eq }}(\mathbf{m})=\frac{1}{\left|\mathbf{C}_{\mathbf{N}}\right|} \sum_{\mathbf{c}_{x, y, z, \xi, \eta, \kappa} \in \mathbf{C}_{\mathbf{N}}} \delta\left(m_{x, y, z}-m_{x+\xi, y+\eta, z+\kappa}\right)
$$

Here, $\delta()$ denotes the Kronecker delta function: $\delta(0)=1$ and 0 otherwise. To identify the second-order MGRF model, only the potential value $V_{\text {eq }}$ need to be estimated.

To compute the second term, $\frac{1}{|\mathbf{R}|} \log P(\mathbf{m})$, in Equation (39) for a region map $\mathbf{m}$, a reduced version of the approximate partition function $Z_{\mathbf{N}}$ in [178] is used (see 
also [253]):

$$
\begin{aligned}
Z_{\mathbf{N}} & \approx \exp \left(\sum_{x, y, z \in \mathbf{R}} \sum_{\xi, \eta, \kappa \in \nu_{s}} \sum_{\lambda \in \mathbf{L}} V\left(\lambda, m_{x+\xi, y+\eta, z+\kappa}\right)\right) \\
& =\exp \left(\left|\mathbf{C}_{\mathbf{N}}\right| \sum_{\lambda \in \mathbf{L}}\left(V_{\mathrm{eq}} f_{\lambda}(\mathbf{m})-V_{\mathrm{eq}}\left(1-f_{\lambda}(\mathbf{m})\right)\right)\right) \\
& =\exp \left(V_{\mathrm{eq}}\left|\mathbf{C}_{\mathbf{N}}\right|(2-L)\right)
\end{aligned}
$$

where $f_{\lambda}(\mathbf{m})$ is the marginal frequency of the label $\lambda$ in the map $\mathbf{m}$. This approximate partition function (which becomes too trivial for $L=2$ ) results in the following approximation of the second term $\frac{1}{|\mathbf{R}|} \log P(\mathbf{m})$ in Equation (39):

$$
\varrho V_{\mathrm{eq}}\left(2 f_{\mathrm{eq}}(\mathbf{m})+L-3\right) \approx 4 V_{\mathrm{eq}}\left(2 f_{\mathrm{eq}}(\mathbf{m})+L-3\right)
$$

where $\varrho=\frac{\left|\mathbf{C}_{\mathbf{N}}\right|}{|\mathbf{R}|} \approx\left|\nu_{s}\right|=4$.

\section{Identification of the second-order MGRF model}

The approximate log-likelihood term in Equation (42) is unsuitable for estimating the model parameter $V_{\text {eq }}$ that specifies the Gibbs potential. Therefore, the second-order MGRF model is identified using a reasonably close first approximation of the maximum likelihood estimate (MLE) of $V_{\text {eq }}$ derived for a given region map $\mathbf{m}^{\circ}$ in accord with [257] from the unconditional log-likelihood $L_{u}\left(\mathbf{m}^{\circ} \mid V_{\mathrm{eq}}\right)=\frac{1}{|\mathbf{R}|} \log P\left(\mathbf{m}^{\circ}\right)$ of Equation (40) with the exact partition function $Z_{\mathbf{N}}=\sum_{\mathbf{m} \in \mathcal{X}} \exp \left(V_{\mathrm{eq}} \varrho|\mathbf{R}|\left(2 f_{\mathrm{eq}}(\mathbf{m})-1\right)\right)$ where $\mathcal{X}$ is the parent population of region maps:

$$
\begin{aligned}
L_{u}\left(\mathbf{m}^{\circ} \mid V_{\text {eq }}\right) & =V_{\text {eq }} \varrho\left(2 f_{\text {eq }}\left(\mathbf{m}^{\circ}\right)-1\right) \\
& -\frac{1}{|\mathbf{R}|} \log \left(\sum_{\mathbf{m} \in \mathcal{X}} \exp \left(V_{\text {eq }} \varrho|\mathbf{R}|\left(2 f_{\text {eq }}(\mathbf{m})-1\right)\right)\right)
\end{aligned}
$$

The approximation is obtained by truncating the Taylor's series expansion of $L\left(\mathbf{m}^{\circ} \mid V_{\mathrm{eq}}\right)$ in the close vicinity of zero potential, $V_{\mathrm{eq}}=0$, to the first three terms:

$$
L_{u}\left(\mathbf{m}^{\circ} \mid 0\right)+\left.V_{\text {eq }} \frac{d L\left(\mathbf{m}^{\circ} \mid V_{\text {eq }}\right)}{d V_{\text {eq }}}\right|_{V_{\text {eq }}=0}+\left.\frac{1}{2} V_{\text {eq }}^{2} \frac{d^{2} L_{u}\left(\mathbf{m}^{\circ} \mid V_{\text {eq }}\right)}{d V_{\text {eq }}^{2}}\right|_{V_{\text {eq }}=0}
$$


Since zero potential produces an independent random field (IRF) equiprobable region labels $\lambda \in \mathbf{L}$, the relative frequency of the equal pairs of labels over $\mathbf{C}_{\mathbf{N}}$ has in this case the mean value $\frac{1}{L}$ and the variance $\frac{L-1}{L^{2}}$. Then the following relationships hold:

$$
\begin{aligned}
& \left.\frac{d L_{u}\left(\mathbf{m}^{\circ} \mid V_{\text {eq }}\right)}{d V_{\text {eq }}}\right|_{V_{\text {eq }}=0}=2 \varrho\left(f_{\text {eq }}\left(\mathbf{m}^{\circ}\right)-\frac{1}{L}\right) \\
& \left.\frac{d^{2} L E_{u}\left(\mathbf{m}^{\circ} \mid V_{\text {eq }}\right)}{d V_{\text {eq }}^{2}}\right|_{V_{\text {eq }}=0}=-4 \varrho \frac{L-1}{L^{2}}
\end{aligned}
$$

where $f_{e q}(\mathbf{m})^{\circ}$ is the relative frequency of the equal label pairs in the region map $\mathbf{m}^{\circ}$ specified in Equation (41). The approximate likelihood of Equation (43) results in the following MLE of $V_{\text {eq }}$ for a given map $\mathbf{m}^{\circ}$ :

$$
V_{\mathrm{eq}}=\frac{L^{2}}{2(L-1)}\left(f_{\mathrm{eq}}\left(\mathbf{m}^{\circ}\right)-\frac{1}{L}\right)
$$

This relationship allows for computing the potentials of the Potts model for each current region map obtained by the Bayesian classification based on the estimated low-level image model. For bimodal images $(L=2)$, the value $V_{\mathrm{eq}}$ is estimated as:

$$
V_{\mathrm{eq}}=2 f_{\mathrm{eq}}\left(\mathbf{m}^{\circ}\right)-1
$$




\title{
CURRICULUM VITAE
}

\author{
Mahmoud Mostapha \\ Paul C. Lutz Hall, Room \# 304 \\ BioImaging Laboratory \\ Department of Bioengineering \\ University of Louisville, Louisville, KY, USA \\ University E-mail:mmmost01@louisville.edu \\ Personal E-mail:mmmosman88@yahoo.com \\ Office: (502) 852-4032 \\ Home: (502) 819-7397
}

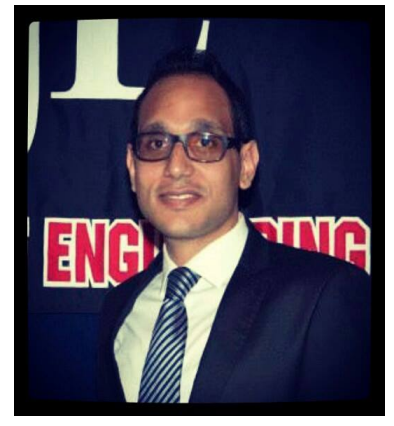

I am a graduate student in the Electrical and Computer Engineering department at the University of Louisville, USA. My general interests are in digital image processing, medical imaging, and computer vision. I am focused on developing new computer aided diagnostic (CAD) systems for the detection, diagnosis and understanding of complex brain disorders (e.g. autism). My primary expertise is in atlas-based medical image segmentation of different MR imaging modalities, especially diffusion tensor imaging (DTI). Since I joined the BioImaging lab in January 2013, my research is concentrated on building a DTI-based CAD system for the early diagnosis of autism that integrates both shape and connectivity extracted features in the classification process. In addition, I have spent a great many years working in the development of computer programming and design patterns and have extensive experience in programming a wide variety of languages on an array of platforms. 


\section{Education}

2014 M.Sc., Electrical Engineering, University of Louisville, Louisville, KY, USA.

M.Sc. Thesis: A Novel DTI-Based CAD System For Early Diagnosis of Autism-Dr. Ayman S. El-Baz and Dr. Tamer Inanc, Thesis Co-advisors. $\mathrm{GPA}=4.0$ (Expected to graduate in summer 2014)

2009 B.Sc., Electronics and Communications Engineering, Mansoura University, Mansoura, Egypt.

Sr. Project: Remotely Controlled Humanoid Robot- Dr. Sherif Kishk Project Supervisor.

$\mathrm{GPA}=4.0$ (Ranked the first in a class of 250)

\section{Experience}

2013-2014 Graduate Research Assistant, BioImaging laboratory, Department of Bioengineering, University of Louisville, Louisville, KY, USA.

2010-2012 Graduate Teaching Assistant, Department of Electronics and Communications Engineering, Mansoura University, Mansoura, Egypt.

\section{$\underline{\text { Professional Affiliations and Training }}$}

- Member, Institute of Electrical and Electronics Engineers (IEEE).

- Member, IEEE Signal Processing (SPS) Society.

- Member, Medical Imaging, Computing and Computer-Assisted Interventions (MICCAI).

- Committee Head, IEEE Student Branch, Mansoura University, Egypt. 


\section{Teaching Experience}

Assist in teaching, laboratory demonstration, conducting tutorials, grading, and senior graduation projects of the following Electrical Engineering undergraduate courses:

\section{Teaching History}

\begin{tabular}{|c|c|c|}
\hline Institution & Place & Semester/Year \\
\hline Mansoura University & $\begin{array}{c}\text { Wireless Communications Systems } \\
\text { Digital Image Processing } \\
\text { Electronic Circuits Laboratory II }\end{array}$ & 2011-2012 \\
\hline Mansoura University & $\begin{array}{c}\text { Computer Networks } \\
\text { Digital Signal Processing } \\
\text { Introduction to Assembly Language }\end{array}$ & $2010-2011$ \\
\hline Mansoura University & $\begin{array}{c}\text { Programming with Matlab for Engineers } \\
\text { Communication Theory } \\
\text { Signals and Systems } \\
\text { Electronic Circuits Laboratory I }\end{array}$ & $2009-2010$ \\
\hline
\end{tabular}

\section{Awards and Recognition}

- University of Louisville Fellowship in the Electrical and Computer Engineering Department, Speed School of Engineering, University of Louisville, April 2014

- Theobald Scholarship Award in the Electrical and Computer Engineering Department, Speed School of Engineering, University of Louisville, April 2013.

- MICCAI 2013 Grand Challenge on MR brain Image Segmentation (MRBRainS13) Winner in the conference of Medical Image Computing and Computer-Assisted Intervention (MICCAI'14), Nagoya, Japan, September 2013.

- Sponsored Research Tuition Award from Spring 2013 through Summer 2014. 
- Young Innovators' Award (YIA) for engineering senior year projects, Egypt, 2010.

- Bibliotheca Alexandrina graduation project Award for engineering senior year projects, Egypt, 2010.

- Higher Education Enhancement Project Fund (HEEPF) Award for outstanding undergraduate students, Egypt, 2006.

\section{Class Work Grade}

A total of 36 credit hours in Electrical Engineering, Mathematics, and Computer Science subjects- cumulative GPA is 4.0. Particular course concentration has been in statistical modeling, medical image analysis, and machine learning. Relevant courses include: digital signal processing, medical image analysis, artificial neural systems, and probability and random variables.

TOFEL Test Scores

\begin{tabular}{cccccc}
\hline \hline Test Date & Reading & Listening & Speaking & Writing & Total \\
\hline OCT 08, 2011 & 23 & 29 & 22 & 24 & 98 \\
\hline
\end{tabular}

GRE Test Scores

\begin{tabular}{ccccc}
\hline \hline Test Date & Verbal & Quantitative & Total & Analytical Writing \\
\hline May 03, 2012 & 146 & 164 & 310 & 4 \\
Jan 28, 2014 & 156 & 163 & 319 & 3.5 \\
\hline
\end{tabular}




\section{Research Activities}

- Autism Diagnosis: Developed a computer-aided diagnostic (CAD) system using diffusion tensor imaging for early diagnosis of autism (programmed in C++/MatLab).

- MR Brain Images Tissues Segmentation: Designed and implemented a complete system to segment different brain tissues using a MAP-based technique for MR brain images based on visual appearance and prior shape (programmed in $\mathrm{C}++$ /MatLab).

- Segmentation of Infant DTI MR Brain Images: Designed and implemented a complete system to segment different brain tissues from infant DTI images using atlas-based approach (programmed in $\mathrm{C}++/$ MatLab).

- Technical Writing: Assisted in grants writing and preparing primary results for the BioImaging Laboratory, University of Louisville, Louisville, KY, USA.

\section{$\underline{\text { Publications }}$}

During my M.Sc. (Spring 2013-Summer 2014), I have authored or co-authored more than 8 technical publications that have appeared in world-renown journals; book chapters; top-rank international conferences and workshops ,including Medical Image Computing and Computer-Assisted Intervention (MICCAI), International Symposium on Biomedical Imaging (ISBI), and International Conference on Image Processing (ICIP). In addition, I have presented a part of the Biolmaging lab MICCAI tutorial entitled Stochastic Modeling for Medical Image Analysis, and was a key member of the BioImaging lab team that represented the University of Louisville at the MICCAI 2013 Grand Challenge titled "MRBRainS13". The challenge pitted major universities from across the globe in designing automated software to be used for segmentation of the brain from Magnetic Resonance Images (MRIs). There were 81 teams registered for this challenge, of which only 12 were invited for the final round of the onsite completion. When the final results were 
tallied, the University of Louisville BioImaging Lab was awarded the first prize for our methods and computational performance. This achievement has garnered both local and international recognition for the university and students involved.

\section{- Journal Articles}

1. A. Alansary, A. Soliman, F. Khalifa, A. Elnakib, M. Mostapha, W. Nitzken, M. Casanova, A. El-Baz, “MAPBased Framework for Segmentation of MR Brain Images Based on Visual Appearance and Prior Shape," MIDAS Journal, 2013 [Online]. Available: http://hdl.handle.net/10380/3440.

\section{- Book Chapters}

1. M. Mostapha, F. Khalifa, A. Alansary, A. Soliman, J. Suri, and A. ElBaz, “Computer Aided Diagnosis Systems for Acute Renal Transplant Rejection: Challenges and Methodologies," Handbook Abdomen and Thoracic Imaging: An Engineering and Clinical Perspective, A. El-Baz, Luca Saba, and J. Suri Eds, Springer-Verlag, New York, 2014, ch. 1, pp. 1-36.

\section{- Peer-Reviewed Conference Proceedings}

1. M. Mostapha, A. Soliman, F. Khalifa, A. Elnakib, A. Alansary, M. Nitzken, M. F. Casanova and A. El-Baz, "A Statistical Framework for the Classification of Infant DT Images," In: Proceedings of IEEE International Conference on Image Processing (ICIP'14), Paris, France, October 27-30, 2014. (Accepted).

2. A. Alansary, A. Soliman, M. Nitzken, F. Khalifa, A. Elnakib, M. Mostapha, M. F. Casanova and A. El-Baz, "An Integrated Geometrical and Stochastic Approach for Accurate Infant Brain Extraction," In: Proceedings of IEEE International Conference on Image Processing (ICIP'14), Paris, France, October 27-30, 2014. (Accepted). 
3. M. Mostapha, A. Alansary, A. Soliman, F. Khalifa, M. Nitzken, R. Khodeir and M. F. Casanova and A. El-Baz, "Atlas-Based Approach for the Segmentation of Infant DTI MR Brain Images," In: Proceedings of IEEE International Symposium on Biomedical Imaging: From Nano to Macro (ISBI'14), Beijing, China, April 29-May 2, 2014, pp. 1255-1258.

4. M. Mostapha, F. Khalifa, A. Alansary, A. Soliman, G. Gimel'farb, and A. El-Baz, “Dynamic MRI-Based Computer Aided Diagnostic Systems for Early Detection of Kidney Transplant Rejection: A Survey," In: Proceedings of International Symposium on Computational Models for Life Science (CMLS'13), Sydney, Australia, November 27-29, vol. 1559, pp. 297-306.

\section{- Peer-Reviewed Presentations and Challenges}

1. M. Mostapha, A. Alansary, A. Soliman, M. Nitzken, A. El-Baz, "Stochastic Modeling for Medical Image Analysis," In: Proceedings of Medical Image Computing and Computer-Assisted Intervention (MICCAI'14), Nagoya, Japan, September 22-26, $2014 . \quad$ Available: https:/ / louisville.edu/speed/bioengineering/faculty/bioengineeringfull/dr-ayman-el-baz/miccai-tutorial.html.

2. M. Mostapha, A. Alansary, A. Soliman, M. Nitzken, A. El-Baz, "MICCAI Grand Challange on MR Brain Image Segmentation (MRBRainS13)," In: Proceedings of Medical Image Computing and ComputerAssisted Intervention (MICCAI'14), Nagoya, Japan, September 22-26, 2014. Available: http://hdl.handle.net/10380/3440. 


\section{$\underline{\text { Technical Skills }}$}

- Programming:

- Matlab, C/C++, Assembly

- Languages:

- Arabic (Native), English (Fluent)

\section{References}

Available when needed. 